Aus der Klinik für Mund-, Kiefer- und Gesichtschirurgie

(Prof. Dr. med. Dr. med. dent. H. Schliephake) im Zentrum Zahn-, Mund- und Kieferheilkunde der Medizinischen Fakultät der Universität Göttingen

\title{
Experimentelle Untersuchung zur Auswirkung für Kopf-Hals-Tumoren relevanter Strahlendosen auf das Randschlussverhalten von Amalgam- und Kunststoff-Füllungen
}

\author{
INAUGURAL - DISSERTATION \\ zur Erlangung des Doktorgrades \\ für Zahnheilkunde \\ der Medizinischen Fakultät der \\ Georg-August-Universität zu Göttingen
}

vorgelegt von

Anne Carolin Gräb

aus

Göttingen 
Dekan:

I. Berichterstatterin: PD Dr. med. dent. S. Sennhenn-Kirchner

II. Berichterstatter/in: PD Dr. Hendrik Wolff

III. Berichterstatter/in:

Tag der mündlichen Prüfung: 30. Juni 2016 


\section{Inhaltsverzeichnis}

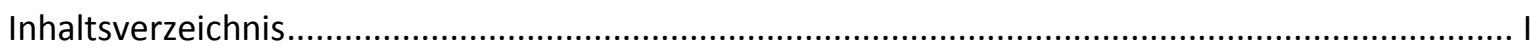

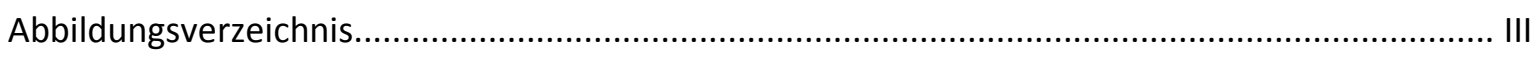

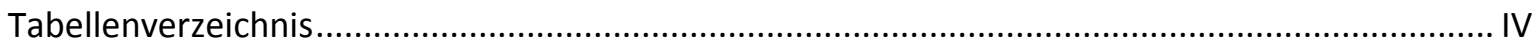

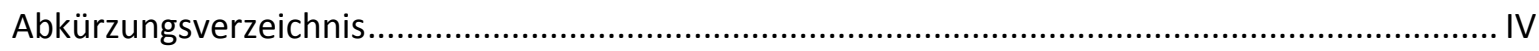

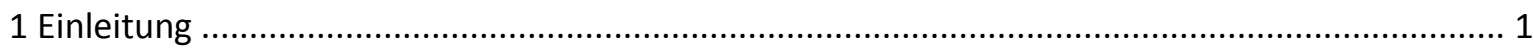

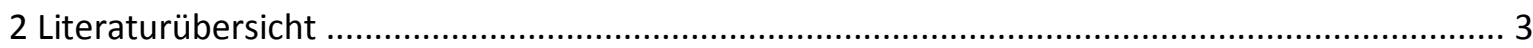

2.1 Grundlagen der Strahlentherapie........................................................................... 3

2.2 Komplikationen und Nebenwirkungen bei Strahlentherapie im Mund-Kiefer-Gesichtsbereich

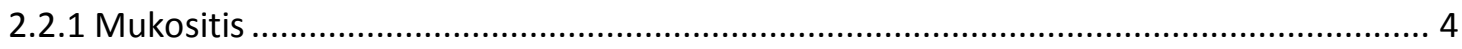

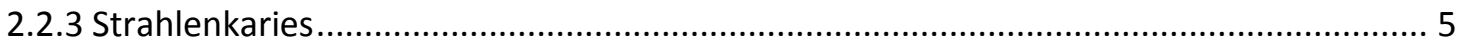

2.2.4 Verlust des Geschmackssinns.................................................................. 7

2.2.5 Kieferklemme ............................................................................................... 7

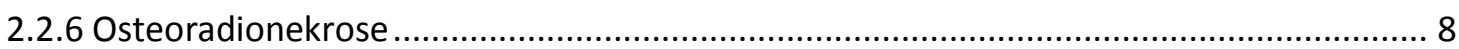

2.3 Umgang und Therapie mit strahlentherapeutischen Patienten in der Zahnarztpraxis............ 9

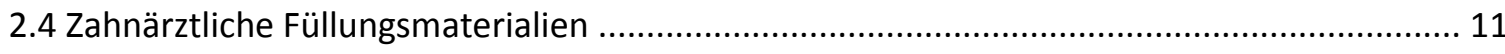

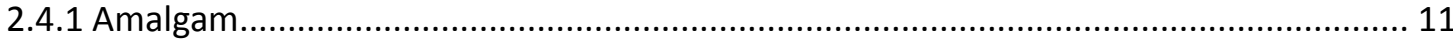

2.4.1.1 Amalgamfüllungen bei Radiotherapiepatienten ............................................12

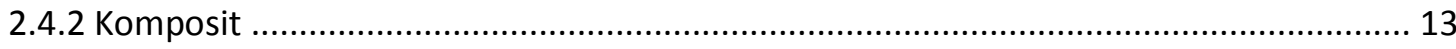

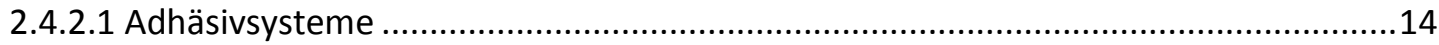

2.4.2.2 Kompositfüllungen bei Radiotherapiepatienten ...........................................15

2.5 Rückstreueffekte von zahnärztlichen Materialien während der Radiotherapie .................... 16

2.6 Auswirkung von Strahlentherapie auf die Zahnhartsubstanz ......................................... 18

2.7 Untersuchungsmethoden von Randspalten an Füllungsmaterialien ................................ 19

2.7.1 Klinisch mit Sonde und Spiegel................................................................. 19

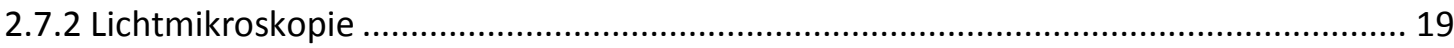

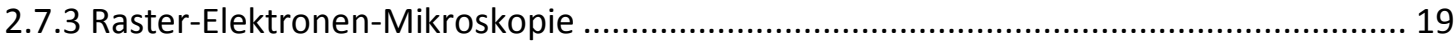

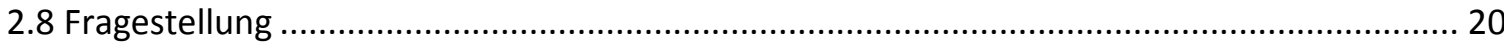

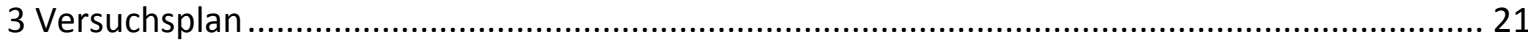

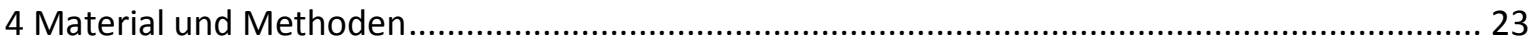

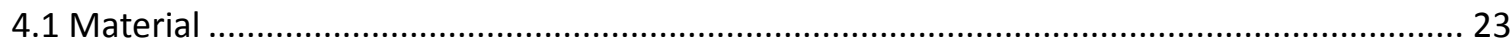

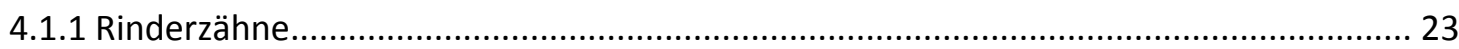

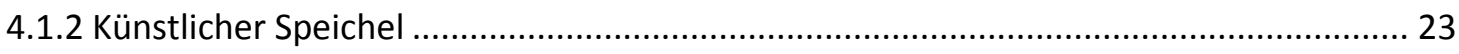

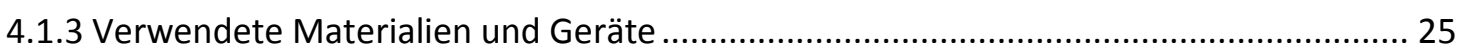




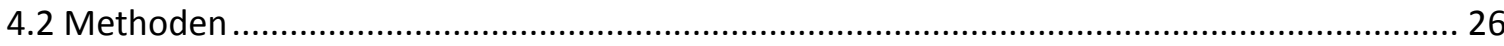

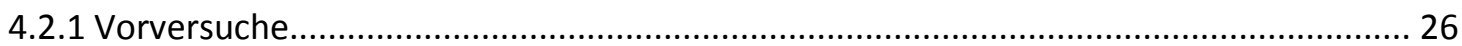

4.2.1.1 Auswirkungen von Strahlentherapie auf Harvardzement, Füllungsmaterialien, Phantommetall, Nickel-Titan-Schlaufen ..................................................................26

4.2.1.2 Bestrahlung der Proben und Mikroskopie ................................................28

4.2.1.3 Versuch der Mikroskopie mittels des Raster-Elektronen-Mikroskops .....................28

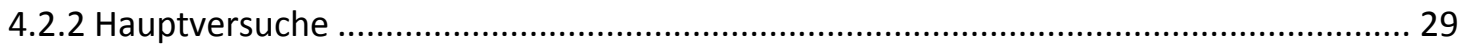

4.2.2.1 Herstellung und Vorbereitung der Rinderzahnproben .........................................29

4.2.2.2 Einbettung der Rinderzähne mit Paladur in einer Gießform ................................30

4.2.2.3 Kavitätenpräparation und Applikation der Füllungsmaterialien Amalgam und

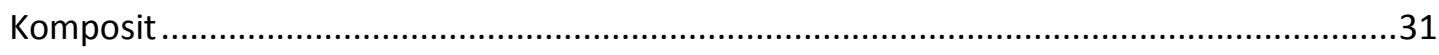

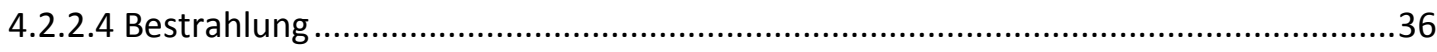

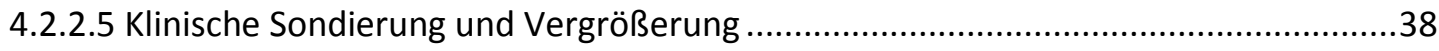

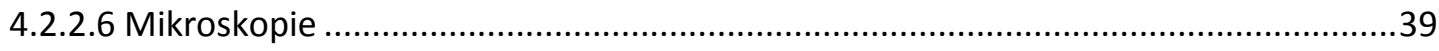

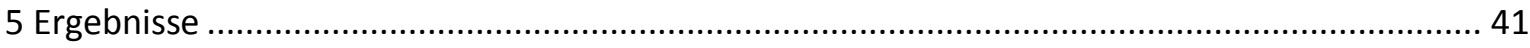

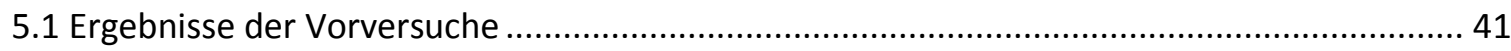

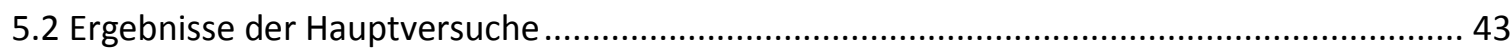

5.2.2 Ergebnisse der Untersuchungen unter dem Auflichtmikroskop.............................. 43

5.2.3 Ergebnisse der klinischen Untersuchung .......................................................... 49

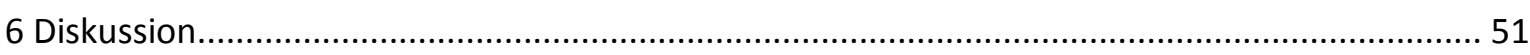

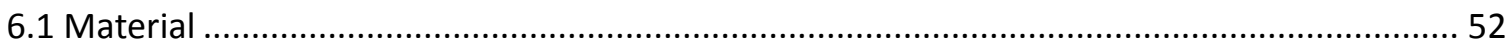

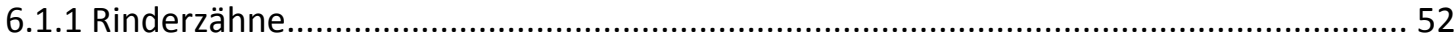

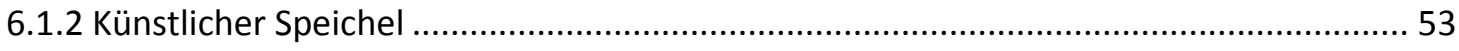

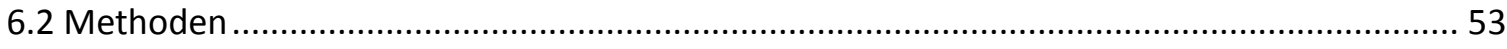

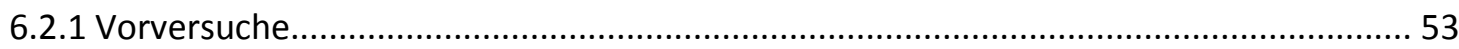

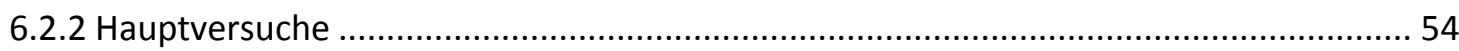

6.2.2.1 Herstellung der Proben............................................................................ 54

6.2.2.2 Auswahl und Verarbeitung der Füllungsmaterialien ............................................55

6.2.2.3 Mikroskopie und Messung der Proben................................................................60

6.2.2.4 Klinische Untersuchung .........................................................................64

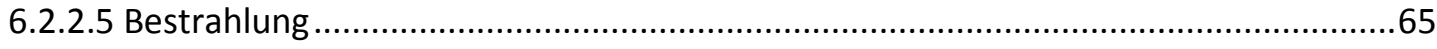

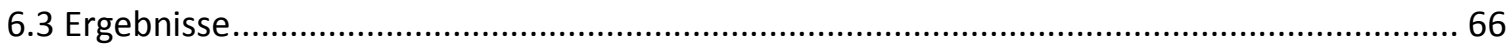

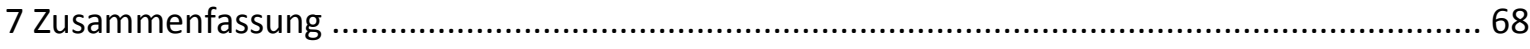

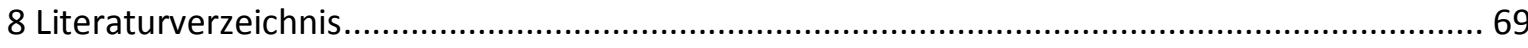




\section{Abbildungsverzeichnis}

Abbildung 1: Speichelsubstanz und die Analysenwaage, mit der sie abgemessen wurde

Abbildung 2: Vorversuche- Gruppe 1: Objektträger mit Harvardzement bestrichen: Bild vor

Bestrahlung

Abbildung 3: Vorversuche- Gruppe 2: Phantommetallplättchen Vergleich unbehandelte Seite:

polierte Seite. Bild vor der Bestrahlung

Abbildung 4: Vorversuche- Gruppe3: Nickel-Titan-Schlaufen mit Harvard-Zement auf

Phantommetallplättchen befestigt. Bild vor der Bestrahlung ....

Abbildung 5: Vorversuche- Gruppe 4: Kavitäten mit Amalgam und Komposit in Rinderzähnen im Silikonblock

Abbildung 6: Probeblock mit Schmelzrissen im Raster-Elektronen-Mikroskop 29

Abbildung 7: Säuberung der Zähne von Verfärbungen mittels eines Scalers............................... 30

Abbildung 8: Kunststoff Paladur ${ }^{\circledR}$, Anrührbecher, Gießform und Schraubzwinge ........................ 30

Abbildung 9: Rinderzähne eingebettet in Kunststoffblöcke .................................................. 31

Abbildung 10: Markierung der Bohrlöcher im Abstand von 5 mm ........................................ 33

Abbildung 11: Block unter der Bohrmaschine Metabo liegend, Markierung des Diamantbohrers in Höhe von $5 \mathrm{~mm}$ zu erkennen ................................................................. 33

Abbildung 12 Betätigung des Hebels und standardisierter Bohrvorgang.................................. 33

Abbildung 13: Gezielte Versenkung des Diamantbohrers bis zu der Markierung ........................ 34

Abbildung 14: Materialien zur Applizierung der Kompositfüllungen ...................................... 35

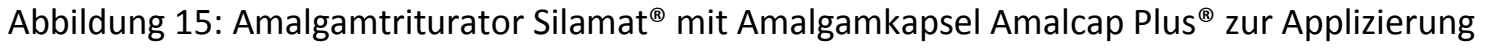
der Amalgamfüllungen

Abbildung 16: Bildschirm der experimentellen Röntgenstrahlanlage mit Bestrahlungszeit, Röhrenspannung, Stromstärke, Filter und Tischhöhe

Abbildung 17: Bestrahlungsindikator zur Anwendung in der Strahlenkammer

Abbildung 18: Unbestrahlter Bestrahlungsindikator.

Abbildung 19: Bestrahlter Bestrahlungsindikator nach Bestrahlung unserer Proben mit 60 Gy .... 37

Abbildung 20: Klinische Untersuchung mit zahnärztlicher Sonde....

Abbildung 21: Probe mit zahnärztlicher Sonde am zu untersuchenden markierten Abschnitt ...... 39

Abbildung 22: Auflichtmikroskop Zeiss Stemi SV11 mit Probe

Abbildung 23: Objektträger mit Zement vor und nach Bestrahlung

Abbildung 24: Phantommetallplättchen unbehandelte/polierte Seite vor und nach Bestrahlung. 42

Abbildung 25: Nickel-Titan-Schlaufe auf Zement befestigt vor und nach Bestrahlung

Abbildung 26: Amalgamfüllung (AgF) in Rinderzahn vor und nach Bestrahlung

Abbildung 27: Kompositfüllung in Rinderzahn vor und nach Bestrahlung 
Abbildung 28: Gruppe 1 Amalgamfüllungen (AgF) mit 60 Gray bestrahlt vor und nach Bestrahlung 44

Abbildung 29: Gruppe 2 Amalgamfüllungen mit 90 Gray bestrahlt vor und nach Bestrahlung ...... 46

Abbildung 30: Gruppe 3 Kompositfüllungen (KoF) mit 60 Gray bestrahlt vor und nach Bestrahlung 47

Abbildung 31: Gruppe 4 Kompositfüllungen mit 90 Gray bestrahlt vor und nach Bestrahlung...... 48

\section{Tabellenverzeichnis}

Tabelle 1: Schematische Darstellung des Versuchsablaufes. 22

Tabelle 2: Zusammensetzung des künstlichen Speichels. .24

Tabelle 3: Zuordnung der Füllungsmaterialien und unterschiedlichen Bestrahlungsdosen in vier Gruppen

Tabelle 4: Anzahl der bei der klinischen Untersuchung nach der Bestrahlung aufgetretenen Veränderungen .49

\section{Abkürzungsverzeichnis}

$\mathrm{Gy}=\mathrm{Gray}$

KoF= Kompositfüllung

AgF= Amalgamfüllung

$\mathrm{GIZ}=\mathrm{Glasionomerzement}$

REM= Raster-Elektronen-Mikroskop 


\section{Einleitung}

Maligne Tumorerkrankungen stellen in Deutschland die zweithäufigste Todesursache dar. Dabei sind weltweit ca. 2 \% aller malignen Tumoren im Bereich der Mundhöhle, der Lippen und des Oropharynx lokalisiert (Ferlay et al. 2010). Hauptsächlich handelt es sich um Plattenepithelkarzinome.

In Deutschland gibt es pro Jahr 9.500 Neufälle bei Männern und 3.500 Neufälle bei Frauen (Krebs in Deutschland 2013). Die Tendenz ist aufgrund der älter werdenden Gesellschaft steigend (Andrews und Griffiths 2001a).

Die Behandlung von Tumoren im Kopf-Hals-Bereich kann neben chirurgischen Eingriffen und einer Chemotherapie auch eine Strahlentherapie erforderlich machen. Die Strahlenbzw. Radiotherapie ist wie bei anderen Tumoren so auch bei fortgeschrittenen Kopf-und Halsplattenepithelkarzinomen ein übliches onkologisches Verfahren, das isoliert oder als kombiniertes Therapiekonzept mit (lasermikro-) chirurgischen und/oder chemotherapeutischen Maßnahmen adjuvant, kurativ wie auch palliativ eingesetzt werden kann (Bornstein et al. 2001; Olthoff et al. 2009; Wolff et al. 2009). Bei der Kombination von Chemound Strahlentherapie, der sog. simultanen Radiochemotherapie, wird u.a. angestrebt, deren jeweilige Wirkung zu steigern, da beide Verfahren unabhängig voneinander die Zelltötung forcieren. Die Chemotherapie kann somit die lokale Wirkung der Strahlentherapie in einer supra-additiven Weise verbessern (Bernier und Bentzen 2003).

Der spezielle Effekt, der von der Strahlentherapie erwartet wird, liegt darin, dass sie die neoplastischen Zellen zerstören kann, während das Normalgewebe zugleich geschont wird. Diese optimale Wirkung kann in der Praxis nicht immer erzielt werden. In der Regel treten zahlreiche unerwünschte Nebenwirkungen auf (Bornstein et al. 2001).

Die Heilungsrate der Patienten mit Tumoren im Kopf-Hals-Bereich hat sich in den letzten Jahren stark erhöht, lag sie vor 50 Jahren noch bei $30 \%$, so ist sie zuletzt auf bis zu $50 \%$ gestiegen (Feyer und Buchali 2007). Diese Verbesserungen sind sowohl auf Weiterentwicklungen in der Diagnostik als auch auf neue Techniken in der Strahlentherapie, wie z.B. die 3D-Bestrahlungsplanung, zurückzuführen (Feyer und Buchali 2007). Dennoch hat die Strahlentherapie im Kopf-Hals-Bereich immer noch gefährliche Nebenwirkungen.

Die Bestrahlung von Tumoren im Kopf-Hals-Bereich hat schädliche Auswirkungen auf die Speicheldrüsen, die Schleimhaut, den Knochen, die Kiefermuskulatur, das Kiefergelenk, 
nicht zuletzt aber auch auf die Zähne. Die Auswirkung der Röntgenstrahlen auf die Mundhöhle und ihre Weichgewebe sowie auf die Zahnhartsubstanzen wurde bereits in zahlreichen Studien untersucht. Besondere Berücksichtigung fand dabei auch schon die durch die rückwärtige Streustrahlung von dichteren Materialien verursachte lokale Dosiserhöhung. Unklar ist immer noch, ob und wie die zahnmedizinischen Materialien bei Patienten mit Kopf-Hals-Tumoren durch die Röntgenstrahlung in ihrer Qualität und Beständigkeit beeinflusst werden. Dieser Forschungslücke gilt die hier vorliegende Untersuchung. Ausgewählte Füllungsmaterialien werden hier darauf untersucht, ob sie durch die in unterschiedlich hoher Dosierung vorgenommene Bestrahlung in ihrer Qualität und Beständigkeit beeinträchtigt werden. 


\section{Literaturübersicht}

\subsection{Grundlagen der Strahlentherapie}

Ziel der Strahlenbehandlung von bösartigen Tumoren ist die Zerstörung der Tumorzellen bei gleichzeitiger Schonung von gesunden Geweben und Organen.

Energiereiche Strahlen wie Röntgenstrahlen werden zur Behandlung dieser malignen Tumoren eingesetzt.

In der Einheit Gray (=Gy) wird die Energiedosis, die von radioaktiven Stoffen ausgehende Strahlung, ionisierende Strahlung genannt, angegeben.

Die ionisierende Strahlung wirkt auf die sich teilenden Zellen. In der Regel reagieren Tumoren, die eine hohe Zellteilungsrate besitzen, hoch sensibel auf diese Strahlung. So wirkt sich die Strahlung auf Tumorzellen, weil sich die Zellteilung bei ihnen meist beschleunigt vollzieht, stärker aus als auf das benachbarte gesunde Gewebe, dessen Zellteilung im Vergleich häufig geringer ausfällt (Schwenzer und Ehrenfeld 2011). Die intelligente Bestrahlungsplanung hinsichtlich der zeitlichen Dosisverteilung ist, da die Proliferationskinetik der Tumorzellen stark variiert, eine der wichtigsten Aufgaben der klinischen Radiotherapie. Für Plattenepithelkarzinome im Kopf-Hals-Bereich liegt die Zeit, in der sich die Zellen verdoppeln, bei nur 3-5 Tagen. Die Fraktionierung, also die zeitliche Verteilung der Gesamtdosis auf kleine Einzeldosen, muss deshalb auf diese Proliferation der Tumorzellen abgestimmt sein. Die konventionelle Fraktionierung und übliche Vorgehensweise in der Radiotherapie bei Patienten mit Tumoren im Kopf-Hals-Bereich beinhaltet Einzeldosen von 1,8-2 Gy, die 5-mal wöchentlich appliziert werden. Dies ergibt eine Wochendosis von 9-10 Gy und eine Gesamtbehandlungszeit von 5-6 Wochen für 45-50 Gy bzw. 6-7 Wochen für 54-60 Gy (Kauffmann et al. 2001).

Durch diese zeitliche Verteilung können Reparaturmechanismen im gesunden Gewebe in Gang gebracht werden, während dagegen die Tumorzellen in der Regel eine schlechtere Reparaturfähigkeit hinsichtlich der DNA-Schäden besitzen.

Je höher die Dosis ist, umso größer ist die Chance auf eine bessere Wirkung. Die kurative Strahlendosis für die Plattenepithelkarzinome (1-3 cm großer Tumor) liegt bei 60-70 Gy (Kauffmann et al. 2001).

Meist handelt es sich heute um eine perkutane Bestrahlungsart. 
Häufig wird die Strahlentherapie im Rahmen eines multimodalen Therapiekonzepts als prä- oder postoperative Maßnahme durchgeführt.

\subsection{Komplikationen und Nebenwirkungen bei Strahlentherapie im Mund-Kiefer- Gesichtsbereich}

Aufgrund von anatomischen und klinischen Gegebenheiten wird bei jeder Strahlentherapie das Normalgewebe in das Bestrahlungsfeld mit einbezogen. Vor allem die Therapie von Tumoren im Kopf-Hals-Bereich bedeutet für die betroffenen Patienten, dass sie mit riskanten Nebenwirkungen rechnen müssen.

Die Nebenwirkungen von Radiotherapie beinhalten u.a. Mukositis, Hyposalivation, Geschmacksverlust, Osteoradionekrosen, Kieferklemme und Strahlenkaries (Dreizen et al. 1977; Rahman et al. 2013; Vissink et al. 2003b).

Ebenfalls muss bei Patienten, die an einem Plattenepithelkarzinom erkrankt sind und behandelt wurden, das hohe Risiko von einem Auftreten maligner Zweittumore beachtet werden (Wolff et al. 2013).

Zu den akuten und reversiblen Nebenwirkungen zählen die Mukositis sowie Geschmacksverluste. Zu Nebenwirkungen, die chronisch verlaufen, zählen die Atrophie der Speicheldrüsen, Strahlenkaries und Osteoradionekrosen (Dörr et al. 2002; Kielbassa et al. 2006; Larson 1986). Einigen hier genannten akuten wie chronisch verlaufenden Nebenwirkungen soll im Folgenden noch genauere Beachtung geschenkt werden.

\subsubsection{Mukositis}

Orale Mukositis ist ein entzündlicher und ulzerativer Prozess in der Mundhöhle, der aus einem Angriff auf die epithelialen Zellen der oropharyngealen Mukosa resultiert. Sie wird zumeist in der Verbindung von Radio- oder Chemotherapie ausgelöst (Cawley und Benson 2005).

Eine Mukositis, die durch Strahlentherapie (unter Umständen in Verbindung mit einer Chemotherapie) ausgelöst wurde, ist gekennzeichnet durch Rötungen und Schwellungen sowie durch später auftretende pseudomembranöse Erosionen. Heilen diese ab, entsteht 
eine fibrotische und atrophische Schleimhaut. Tritt zudem eine Xerostomie auf, ist die Gefahr der Sekundärinfektion mit Candida albicans besonders hoch (Reichart 2002).

Hinzuweisen ist in unserem Zusammenhang auch darauf, dass die orale Mukositis bereits durch die rückwärtigen Streustrahlen, die von metallischen Materialien ausgehen, entstehen kann (Farahani et al. 1993; Gibbs et al. 1976; Mail et al. 2013; Reitemeier et al. 2002).

Die negativen Folgen, die mit einer oralen Mukositis einhergehen, führen zu einer verzögerten Behandlung, zu einer geringeren einsetzbaren - und damit weit weniger kurativen - Strahlendosis, zu einem veränderten Ernährungsverhalten, zu Dehydrierung und starken Schmerzen (Farrington et al. 2010).

Noch immer dürfte allerdings von einer defizitären Diagnostik der Mukositis zu reden sein und damit auch von Hindernissen beim Vorankommen in der Prävention und im Umgang mit ihr (Eilers und Epstein 2004).

\subsubsection{Xerostomie und Speicheldrüsenfehlfunktion}

Xerostomie oder Mundtrockenheit gehört zu den häufigsten Nebenwirkungen der Radiotherapie bei Kopf- und Halstumoren (Murdoch-Kinch und Zwetchkenbaum 2011). Die Speicheldrüsen reagieren sehr empfindlich auf radiogene Strahlung. Schon in den ersten Wochen kommt es zu einer Erniedrigung der Speichelflussrate bei einer normalen Strahlendosis von z.B. 2 Gy/Tag. Der Speichelfluss wird im Laufe der Therapiedauer immer geringer, vor allem wenn beide Parotiden betroffen sind (Möller et al. 2004).

Tatsächlich erleiden bis zu 64 \% der Patienten, die eine Radiatio im Kopf-Hals-Bereich bekommen haben, einen mittelschweren bis schweren Grad der Xerostomie, - wenn man sie bis zu 22 Jahre danach untersucht (Wijers et al. 2002).

\subsubsection{Strahlenkaries}

Um die Ursachen der Strahlenkaries genau zu verstehen, müssen die verschiedenen Einflüsse der Radiotherapie, wie sie hier zum Teil jetzt schon angesprochen wurden, auf die orale Mukosa, die Speicheldrüsen und eben auch die Zähne betrachtet werden (Kielbassa 
et al. 2006). Die radiogene Karies kann ganz plötzlich entstehen und dabei zum Passungsverlust von Kronen oder auch zu völligem Zahnverlust führen.

Als Folge einer Radiotherapie bei Tumoren im Mund-Kiefer-Gesichtsbereich kann eine vollständige oder teilweise Zerstörung der Speicheldrüsen auftreten. Daraus entstehende Folgen sind ein verminderter Speichelfluss (Xerostomie) und eine Veränderung der Zusammensetzung des Speichels (Proteine, Elektrolyte u.a.). Die wichtigen Funktionen des Speichels wie die Schutz- und Pufferfunktion sowie die Remineralisationswirkung gehen verloren. Gleichzeitig kommt es zu einer Besiedlung der Mundhöhle mit zahlreichen kariogenen Mikroorganismen. Folglich entstehen extrem kariogene Bedingungen, die eine rasche Kariesentstehung und -progression erleichtern (Hellwig et al. 2009). Die Ursache für die Schädigung der Zähne nach Strahlentherapie wird von vielen Autoren ganz allgemein als ein indirekter Effekt angesehen, der hauptsächlich durch die zahlreichen strahlenabhängigen Nebenwirkungen, insbesondere den verminderten Speichelfluss, ausgelöst wird (Jansma et al. 1988b). Es wird aber auch die direkte Wirkung der Strahlen auf die Zähne diskutiert, vor allem angesichts der raschen Progression. Diese direkte Wirkung wiesen Grötz und Mitarbeiter (Grötz et al. 1997) nach. Sie zeigten, dass es zu einer Obliteration der Odontoblastenfortsätze kam und daraufhin auch zu einer Obliteration der Dentinkanälchen. Dies scheint das Resultat eines direkten radiogenen Zellschadens mit Ernährungsstörungen im Bereich der Odontoblastenendigungen zu sein.

Das klinische Erscheinungsbild der Strahlenkaries kann in vier Klassen unterteilt werden:

1.) generalisierte oberflächliche Defekte bis zur Frakturierung und Ablösung des Schmelzes,

2.) der Wechsel der Zahnfarbe zu braun-schwarz,

3.) langsames "Abschmelzen" der Schneidekanten und okklusalen Flächen, 4.) oberflächliche Zerstörung der Zahnhälse (Thiel 1989b).

Die Strahlenkaries gilt als eine lebenslange Bedrohung für die Patienten, die eine Strahlentherapie bekommen haben. Diese müssen lebenslang eine adäquate Mundhygiene und regelmäßige Fluoridapplikation betreiben (Vissink et al. 2003a).

Hyposalivation und Strahlenkaries sind aus zahnärztlicher Sicht die Hauptspätkomplikationen. Die Dokumentation und Erhebung der späten Strahlenfolgen an den Zahnhartge- 
weben wie Initialläsionen an Prädilektionsstellen sind, im Gegensatz zu ihren frühen Strahlenwirkungen, sehr bedeutend für die rechtzeitige Therapieeinleitung (Grötz et al. 2001).

\subsubsection{Verlust des Geschmackssinns}

Die Veränderung der Geschmacksempfindung taucht als ein Resultat des direkten Effekts der Radiatio auf die Geschmacksknospen und die Veränderung der Speichelflüssigkeit auf (Spielman 1998; Mossman 1986).

Schon eine leichte Verminderung des Speichelflusses führt zu einer Verminderung der Anzahl der Geschmacksknospen. Wahrscheinlich ist die Hyposalivation auch so für eine Veränderung der Form und Funktion der restlichen Geschmacksknospen verantwortlich (Henkin et al. 1972).

In den meisten Fällen ist der Geschmacksverlust nur temporär und kehrt innerhalb eines Jahres nach der Radiatio auf ein gering vermindertes oder sogar völlig normales Niveau zurück (Tomita und Osaki 1990).

\subsubsection{Kieferklemme}

Die Kieferklemme kann während der Radiatio, vor allem bei einer Tumorinfiltration in die Muskulatur und der mit ihr verbundenen Operation, als eine einschränkende Nebenwirkung auftreten. Einer der entscheidendsten Faktoren beim Auftreten einer Kieferklemme ist wohl die Miteinbeziehung des M.pterygoideus medialis in das Bestrahlungsfeld (Goldstein et al. 1999).

Schon vor Beginn der Radiotherapie sollte daher die Mundöffnung gemessen werden. Falls sich während der Behandlung geringere Werte ergeben, sollten den Patienten Übungen gezeigt werden, mit denen sie die Muskulatur trainieren, damit sich die Mundöffnung nicht weiter vermindert (Engelmeier und King 1983).

In schweren Fällen können eine Einschränkung der Nahrungsaufnahme und eine Verschlechterung der Mundhygiene die Folgen sein. 


\subsubsection{Osteoradionekrose}

Die Osteoradionekrose ist eine der schwerwiegendsten Komplikationen der Radiotherapie bei Kopf-und Halstumoren. Mit diesem Risiko ist bei 4-35 \% der Patienten zu rechnen (Thiel 1989a).

Die Strahlentherapie hat eine starke Beeinträchtigung des Knochenstoffwechsels im betroffenen Gebiet zur Folge (Kielbassa 2004).

Als Ursache gilt eine durch Strahlen verursachte Gefäßschädigung. Sie führt über Endarteriitis, Hyalinisierung und Thrombosierung zu einer Gefäßfibrose (Marx 1983). Es kommt zu einem Verlust an Osteozyten, aktiven Osteoblasten und Osteoklasten, damit zu einem Verlust des normalen Knochenstoffwechsels und einer Abschwächung des Regenerationsund Heilungsprozesses (Bonan et al. 2006; Maier et al. 2000; Thiel 1989a). Bei den leichtesten Verletzungen der oralen Mukosa können Mikroorganismen in den Knochen eindringen und sich ungehindert ausbreiten. Der strahlengeschädigte und schlecht durchblutete Knochen ist folglich einer enorm hohen Infektionsgefahr ausgesetzt.

Die Osteoradionekrose tritt fast ausschließlich im Unterkiefer auf (Beumer und Brady 1978), denn in der Mandibula erfolgt die Versorgung in Gestalt einer funktionellen Endarterie durch die A. alveolaris inferior. Im Unterschied zum Oberkiefer fehlen hier größere Gefäßanastomosen.

Es gilt zwei Formen der Osteoradionekrose zu unterscheiden. Bei der aseptischen Osteoradionekrose bleibt der devitale Knochen lange Zeit symptomlos. Bei der zweiten Form, der septischen Osteoradionekrose, auch Radio-Osteomyelitis genannt, kann der Patient starken Schmerzen oder anderen erheblichen Beeinträchtigungen ausgesetzt sein (Kielbassa 2004).

Zu den Risiko- und Promotionsfaktoren gehören das Vorhandensein von kariösen oder parodontal geschädigten Zähnen, chirurgische Eingriffe (vor/nach Radiatio), Alkohol- und Nikotinabusus, Bestrahlungsdosis, Art der Strahlentherapie, Bestrahlungsfeld und Stadium des Tumors (O'Dell und Sinha 2011; Thiel 1989a). Bei einer Dosis von über $70 \mathrm{~Gy}$ wurde in $9 \%$ der Fälle eine Osteoradionekrose festgestellt (Bedwinek et al. 1976).

Ausgewählte Extraktionen vor der Radiotherapie steigerten die Fälle der Osteoradionekrose, während Zahnerhaltungsmaßnahmen das Auftreten der Osteoradionekrosen ver- 
minderten. Häufigeres Auftreten war bei Tumoren zu beobachten, die sich nahe des Knochens befanden (Bedwinek at al. 1976).

In fortgeschrittenen oder beständigen Fällen von Osteoradionekrosen bleibt die chirurgische Behandlung die einzige Option, eingeschlossen mikrovaskulärer rekonstruktiver Techniken für Knochen und Weichgewebe (Rice et al. 2015).

\subsection{Umgang und Therapie mit strahlentherapeutischen Patienten in der Zahnarztpraxis}

Der Umgang mit und die Betreuung von bestrahlten Patienten mit Tumoren im Kopf-HalsBereich ist eine besondere Herausforderung für die interdisziplinäre Zusammenarbeit von Zahn- und Humanmedizin (Koga et al. 2008; Sennhenn-Kirchner et al. 2009). Zusätzlich zur engen Kooperation zwischen den Chirurgen und Zahnärzten sollte die Konsultation eines Radiotherapeuten gehören (Reitemeier et al. 2002).

Der Zahnarzt spielt eine entscheidende Rolle bereits bei der Prävention (Moore et al. 2012). Zu den präventiven Maßnahmen gehören u.a. das Scaling und die Politur sowie Mundhygieneinstruktionen von der regelmäßigen und richtigen Zahnputztechnik über die Nutzung von Zahnseide bis hin zu Ernährungsempfehlungen wie z.B. der Reduktion der Zuckerzufuhr (Andrews und Griffiths 2001b). Auch sollten die Patienten in jeder Behandlung einer eingehenden systematischen Weichgewebsuntersuchung unterzogen werden (Moore et al. 2012). Auf alle Fälle sollte der Zahnarzt hinzugezogen werden, sobald eine Tumordiagnose im Kopf-, Halsbereich gestellt wurde (Mealey et al. 1994), um z.B. potenzielle Herde für Infektionen noch vor Beginn der Radiatio zu eliminieren. Während der Strahlentherapie sollte der Zahnmediziner für die Vorbeugung und Linderung der aufgetretenen Symptome wie Mukositis, orale Candidiasis oder zur Vorbeugung von Trismus verfügbar sein. Auch im Anschluss an die Radiatio sollte der Zahnarzt regelmäßige Recalls einführen, um den Schweregrad der Strahlenkaries und Xerostomie beobachten und eventuell auftretende Post-Osteoradionekrosen entdecken zu können (Murdoch-Kinch und Zwetchkenbaum 2011; Moore et al. 2012).

In der heutigen Zeit sucht auch fast jeder Patient eine Zahnklinik vor seiner Strahlentherapie auf und erfährt eine individuelle Therapie. Im Vergleich zu früher bekommt heute auch z.B. jeder Patient Fluoridierungsschienen angefertigt. Es wurden jedoch nur $53 \%$ der 
Patienten auch nach einer Radiotherapie noch ausreichend zahnärztlich betreut (Sennhenn-Kirchner et al. 2009).

Patienten, die eine Radiotherapie bekommen haben, haben die höchsten DMFT-Indizes (Abkürzung für defekte bleibende Zähne: Decayed Missing Filled Teeth) verglichen mit den Patienten, die sich einer Chemotherapie unterziehen mussten (Hong et al. 2010). Dies fand die Arbeitsgruppe um Hong et al. (2010) in ihrem Review heraus, wobei sie ebenfalls feststellten, dass die höchste Kariesprävalenz bei Patienten in der PostChemotherapie bestand. Dies führten sie u.a. auf die umfangreichen und teilweise "aggressiven" Sanierungsmaßnahmen bei Strahlentherapiepatienten zurück.

Die Nutzung von Fluoriden und Chlorhexidin (CHX) sind von stark positivem Nutzen bei Patienten in der Post-Radiotherapie (Sennhenn-Kirchner et al. 2009). Sie reduzieren die Kariesaktivität und Plaqueindizes (Hong et al. 2010).

Durch das hohe Vorkommen an Zweittumoren sollten zusätzlich zu den RoutineNachsorgen auch Computertomographien des Thorax stattfinden, um bei den Patienten Zweittumore erkennbar zu machen. 86 \% der untersuchten Patienten, bei denen sich ein Zweittumor bestätigt hat, konnten kurativ behandelt werden (Wolff et al. 2013).

Die Osteoradionekrose ist die häufigste und schwerwiegendste Spätkomplikation der Radiatio, die in 60-90 \% der Fälle in Folge dentogener Ursachen, wie Extraktionen, auftritt (Curi et al. 1997; Grötz et al. 2001). Angesichts der zu treffenden Entscheidung, die Zähne vor oder nach der Radiotherapie zu extrahieren, sollten die individuellen Eigenschaften des Patienten, die Beschaffenheit des Tumors und der Verlauf der onkologischen Behandlung, wie z.B. Höhe der Dosis und Anzahl der Zähne, die im Strahlenfeld liegen, mit einbezogen werden (Koga et al. 2008). Die gründliche Einschätzung des Zahnstatus und der Compliance der Patienten vor der Radiotherapie kann die Komplikationen reduzieren, wenn sie dazu führt, dass nur Zähne im fragwürdigen parodontalen, endodontalen oder nicht mehr restaurierbaren Zustand bei Patienten mit einer schlechten Compliance extrahiert werden (Koga et al. 2008; Murdoch-Kinch und Zwetchkenbaum 2011; Vissink et al. 2003a). So stellten auch Horiot et al. (1983) fest, dass die Erhaltung der Zähne von bestrahlten Patienten die Regel und nicht die Ausnahme sein sollte.

Dies sind durchaus weniger radikale Ansichten, als sie von Herzog et al. (1986) geäußert wurden, nach denen die „Indikation zur radikal chirurgischen Zahnsanierung bei Mali- 
gnomen im Kopf-Hals-Bereich umso großzüger gestellt werden sollte" und eine konservative Behandlung nur in den seltensten Fällen in Erwägung zu ziehen sei.

Es sollte aber auf alle Fälle bei stattgefundener Extraktion ein sorgfältiger Wundverschluss der Extraktionsalveolen vollzogen werden, wobei idealerweise bis zum Beginn der Radiotherapie 10-14 Tage vergehen sollten (Regezi et al. 1976). Nach der Arbeitsgruppe um Regezi et al. (1976) wurde auf Grund solcher Maßnahmen im Zusammenhang von 311 Extraktionen bei 49 Patienten, die sich unmittelbar vor einer Radiotherapie befanden, nur bei einem Patienten anschließend eine Osteoradionekrose entwickelt.

So ist die interdisziplinäre und konzeptionelle Zusammenarbeit zwischen Strahlentherapeuten, Onkologen, Mund-Kiefer-Gesichtschirurgen, Hals-Nasen-Ohrenärzten, Zahnärzten in Zahnkliniken sowie in Einzelpraxen geboten (Sennhenn-Kirchner et al. 2009). Wenn aber die Extraktionen nach der Radiatio doch unvermeidbar sein sollten, sollten diese von chirurgischen Spezialisten mit den entsprechend notwendigen Techniken und begleitenden Nachsorgen durchgeführt werden (Koga et al. 2008).

Nach Stellungnahme der DGZMK wird der Umfang der Extraktion stets kontrovers diskutiert und die für die Therapiefestlegung wichtigen Parameter unterscheiden sich. Die selektive Extraktions-Indikation wird aber von vielen Autoren empfohlen (DGZMK- Wissenschaftliche Stellungnahme 2002).

Angesichts der demographischen Entwicklung und den mit der Strahlentherapie einhergehenden lebenslangen Nebenwirkungen werden die Zahnärzte in Zukunft mit einem umfangreichen, aus der Strahlentherapie erwachsenden Behandlungsbedarf konfrontiert werden (Moore et al. 2012).

\subsection{Zahnärztliche Füllungsmaterialien}

\subsubsection{Amalgam}

Seit weit mehr als einem Jahrhundert wird Amalgam als Füllungsmaterial verwendet (Rasines Alcaraz et al. 2014). Amalgam entsteht, wenn Quecksilber und Feilungspulver (sogenanntes Alloy) miteinander vermischt werden (Hellwig et al. 2009).

Alle ästhetisch vorteilhafteren Alternativen zu Amalgam erfordern ein komplexeres Prozedere und bedeutend mehr Zeitaufwand. Außerdem ist Amalgam immer noch das ge- 
eignetste Material für Restaurationen im Molarenbereich, wenn Kosten einen Gesichtspunkt darstellen (Mitchell et al. 2007; Roulet 1997).

Auch wenn die Verwendung von Amalgam deutlich zurück gegangen ist, ist es in großen Teilen der Welt das meist verwendete direkte Restaurationsmaterial für die kaukräftetragenden Molaren (Mitchell et al. 2007; Rasines Alcaraz et al. 2014).

An Amalgam, das zu etwa $50 \%$ aus Quecksilber mit der Ordnungszahl 80 besteht, kann eine Streustrahlung entstehen, die einer überwiegend aus Gold bestehenden Legierung mit der Ordnungszahl 69 annähernd äquivalent ist.

\subsubsection{Amalgamfüllungen bei Radiotherapiepatienten}

Eine Studie von Haveman und Mitarbeitern (Haveman et al. 2003) untersuchte das Auftreten von Sekundärkaries an Amalgam- und Glasionomerzementfüllungen (GIZ) bei Strahlentherapiepatienten, die an Xerostomie litten. Im Vergleich wurde bei den Patienten mit Amalgamfüllungen, die nicht regelmäßig Fluoride benutzten, häufiger Karies an den Füllungsrändern diagnostiziert als bei den Patienten mit dem fluoridfreisetzenden Glasionomerzement als Füllungsmaterial. Es wird angenommen, dass Fluorid-freisetzende Materialien Sekundärkaries bei Hochrisikopatienten mit Xerostomie, die nicht regelmäßig Fluorid-Gel anwenden, reduzieren. Bei Patienten, die regelmäßig Fluorid-Gel anwendeten, waren keine Kariesrezidive auszumachen. Zahnmediziner sollten folglich die Patienten stets animieren regelmäßig Fluorid-Gel zu verwenden.

In einer ähnlichen Studie von Wood et al. (1993) wurden Patienten mit Kopf-HalsTumoren mit Glasionomerzementfüllungen und Amalgamfüllungen versorgt. Alle Patienten litten an Xerostomie. Bei Patienten, die täglich eine mittelsaure $(\mathrm{pH} \mathrm{5,8)} \mathrm{Portion} \mathrm{Fluo-}$ ridgel anwendeten, versagten die Glasionomerzementfüllungen, aber nicht die Amalgamfüllungen. Bei den Patienten, die auf die empfohlene Fluoridapplikation verzichteten, zeigten wiederum die Glasionomerzementfüllungen bessere klinische Ergebnisse. Die mittlere Zeit, in der die Restaurationen verloren gingen, betrug 8,5 Monate. Bei zahlreichen Xerostomiepatienten wurden ähnliche Resultate beobachtet (Wood et al. 1993).

Auch sind schwere Schleimreaktionen neben metallischen Füllungsmaterialien während der Radiotherapie zu beobachten (Mail et al. 2013). 


\subsubsection{Komposit}

Komposit von lateinisch compositium= "zusammengestellt" bedeutet dem Wortsinn nach zusammengestellte Werkstoffe.

„In der Zahnmedizin werden unter Kompositen zahnfarbene, plastische Füllungswerkstoffe verstanden, die nach Einbringen in eine Kavität chemisch oder durch Energiezufuhr aushärten." (Hellwig et al. 2009)

Die drei Hauptbestandteile der modernen Komposite bestehen aus der organischen Matrix, der dispersen Phase (Füller) und der Verbundphase (Silane, Kopolymere). Diese beeinflussen die materialspezifischen Eigenschaften. Im nicht ausgehärteten Zustand sind der Matrix zusätzlich Stabilisatoren, Inhibitoren, Initiatoren, Pigmente und weitere Additiva hinzugefügt. Die Radioopazität wird außerdem durch Zugabe von Füllkörpern mit Schwermetallen wie Barium oder Strontium erzielt.

Die heute allgemein bekannten Einteilungsmöglichkeiten beziehen sich auf die Konsistenz, die enthaltenen Füller und die Basischemie (Matrix). Die Art und die Größe der zugesetzten Füllkörper grenzen die klassischen Kompositmaterialien voneinander ab.

\section{$\underline{\text { Klassische Kompositmaterialien }}$}

\section{1.) Makrofüllerkomposite}

Die konventionellen Komposite enthalten Makrofüller Keramik, Glas oder Quarz zwischen einer Größe von 1-100 $\mu \mathrm{m}$. Sie weisen jedoch wegen ihres hohen Füllstoffanteils (bis 80 Gew.-\%) eine schlechte Polierbarkeit und schlechte optische Eigenschaften auf.

\section{2.) Mikrofüllerkomposite}

Sie enthalten Füllstoffe, deren Größe unter $1 \mu \mathrm{m}$ liegt. Als Füllkörper dienen feine Kieselsäuren(Siliziumdioxid). Da sie nur 50 Gew.-\% Füllkörperanteil besitzen, kommt es zu schlechteren physikalischen Eigenschaften wie z.B. zu einer erhöhten Polymerationsschrumpfung und geringeren Biegefestigkeit.

3.) Hybridkomposite

Bei diesem Kompositmaterial sind etwa 85-90 Gew.-\% der Füllkörper Makrofüller und 1015 Gew.-\% Mikrofüller. Die Hybridkomposite vereinen die Vorteile sowohl der Makrofüller- als auch der Mikrofüllerkomposite. Sie sind gut abrasionsbeständig, gut polierbar und 
ihre Polymerisationsschrumpfung konnte minimiert werden. Je nach Korngröße können sie weiter unterteilt werden in Fein-, Feinst- und Subpartikelhybridkomposite.

Die Hybridkomposite stellen einen Großteil der Komposite auf dem Markt dar, z.B. ist das häufig und auch in dieser Studie verwendete Venus ${ }^{\circledR}$ ein Feinstpartikelhybridkomposit.

\subsubsection{Adhäsivsysteme}

Auch bei modernen Kompositen kommt es bei der Aushärtung zu einem Volumenverlust und der Entstehung eines Randspaltes.

Zum Aufbau des dauerhaften Verbundes zwischen der hydrophilen Zahnhartsubstanz und dem hydrophoben Kompositmaterial wurden Adhäsivsysteme entwickelt.

Der Schmelz wird vor Applikation dieser Adhäsivsysteme und des Komposits mit einer Anschrägung versehen, um die Ätzwirkung und so die Benetzbarkeit des Schmelzes zu verbessern. Die Mikro-Zugfestigkeit an quer/parallel (ca. $45 \mathrm{Grad}$ ) angeschliffenen Schmelzprismen ist höher als an senkrecht zur Prismenlängsachse geschnittenen Schmelzprismen (Ikeda et al. 2002).

Die Adhäsivtechnik reduziert, aber beseitigt nicht vollständig das marginal Leakage (Kóhalmi et al. 1999).

Aktuelle Adhäsivsysteme werden unterteilt nach Etch-and-rinse- und Self-etch-non-rinse Systemen. Bei der Etch-and-rinse-Technik wird separate Phosphorsäure angewandt, um die Hartsubstanz zu behandeln und anschließend die weiter oberflächenkonditionierenden Primer und Adhäsive zu applizieren. Etch-and-rinse-Systeme werden als Zwei- oder Drei-Schritt-Systeme auf dem Markt angeboten, je nachdem ob Primer und Adhäsiv separat oder in einer Flasche kombiniert sind. Hier gegenübergestellt beinhalten Self-etchSysteme säurehaltige Monomere, welche den Zahn simultan ätzen und primen. Sie sind im Handel erhältlich als Zweischritt-oder sogar Einschrittsysteme (Ozer und Blatz 2013). Der Haftverbund von Kompositfüllungsmaterialien zum Schmelz war nach Frankenberger und Tay (2005) deutlich besser nach der Ätzung mit Phosphorsäure. Etch-and-rinseSysteme, wie sie auch in dieser Studie verwendet wurden, sowie Zwei-Schritt-self-etchAdhäsive zeigten eine gute Randadaption zum Dentin und haben eine weit bessere klinische Prognose als Ein-Flaschensysteme (Frankenberger und Tay 2005). 
Die unterschiedlichsten wissenschaftlichen Beweise, die heute zu finden sind, vertreten die Auffassung, dass die Wahl zwischen Etch-and-rinse und Self-etch-Systemen oft eine Sache der persönlichen Präferenz sei (Ozer und Blatz 2013).

\subsubsection{Kompositfüllungen bei Radiotherapiepatienten}

Es existieren kontroverse Meinungen darüber, ob weniger Sekundärkaries an Glasionomerzementfüllungen auszumachen ist als bei anderen Materialien. Hierzu wurde von McComb und Mitarbeitern (McComb et al. 2002) das Auftreten von Sekundärkaries bei verschiedenen Füllungsmaterialien bei Xerostomie-Patienten, die sich einer Radiotherapie unterziehen mussten, untersucht. Auch in dieser Studie wurde festgestellt, dass die Patienten, die eine schlechte Compliance in der Fluoridnutzung bewiesen, materialabhängig eine höhere Rate an Kariesrezidiven entwickelten. Die Gruppe der Patienten, die kein Fluorid nutzen, zeigten statistisch signifikante Unterschiede auf, indem bei ihren GIZFüllungen weit weniger Sekundärkaries zu beobachten war als bei den Patienten der gleichen Gruppe, die mit Kompositen versorgt wurden. Die Kariesrezidive wurden bei den GIZ-Füllungen im Vergleich zu den Kompositfüllungen um bis zu 80 \% reduziert (McComb et al. 2002).

In der Studie von De Moor et al. (2011) wurde das klinische Erscheinungsbild von adhäsiven Füllungsmaterialien bei Patienten mit Xerostomie und hohem Kariesrisiko nach Strahlentherapie untersucht.

Diese Patienten sollten ebenfalls täglich ein neutrales 1 \% Natriumfluoridgel auf ihre Zähne auftragen. Nach 6, 12, 18 und 24 Monaten wurden die Füllungen erneut auf Materialbeständigkeit bzw. -verlust, marginale Adaption und Karies untersucht. Das GIZ stellte erneut eine klinische Karieshemmung bereit, wurde aber leicht abradiert. Das Komposit dagegen gewährleistete die größere strukturellere Integrität (De Moor et al. 2011).

In einer weiteren Studie von Bulucu und Mitarbeitern (Bulucu et al. 2009) wurde die Auswirkung von Strahlentherapie auf das Microleakage von unterschiedlichen Adhäsivsystemen in vitro untersucht. Innerhalb der Grenzen dieser Studie wurde festgestellt, dass die Bestrahlung keine Auswirkung auf das Microleakage von Adhäsivsystemen hat (Bulucu et al. 2009). 
Die Arbeitsgruppe um Biscaro (Biscaro et al. 2009) dagegen zeigte, dass die Bestrahlung einen dosisabhängigen nachteiligen Effekt auf den Verbund von Kompositrestaurationen zum Dentin verursacht.

Ganz unabhängig von der Radiotherapie sind die Bondinghaftkräfte zum Schmelz deutlich höher als zum Dentin (Naves et al. 2012). Nach Naves et al. hat die Strahlentherapie, die bei den Patienten stattfand bevor restaurative Maßnahmen ergriffen wurden, ebenfalls deutlich die Klebehaftung auf beiden Substraten herabgesetzt.

Die Röntgenstrahlen hatten folglich einen deutlich nachteiligen Effekt auf die Haftfestigkeit von Füllungsmaterialien zu Schmelz und Dentin, sofern die adhäsiven Restaurationen nach der Radiotherapie angefertigt wurden (Naves et al. 2012).

\subsection{Rückstreueffekte von zahnärztlichen Materialien während der Radiotherapie}

Wenn die Strahlung in der Mundhöhle durch unterschiedlich dichte Materialien und an metallische Oberflächen dringt, tritt eine rückwärtige Streustrahlung auf (Gagnon und Cundiff 1980). Durch die Rückstreuung der Elektronen können metallische zahnärztliche Materialien wie Implantate, Kronen, Füllungen oder Rekonstruktionsplatten das Strahlenfeld so verändern, dass sich die Strahlendosis der angrenzenden Schleimhaut in höheren Werten darstellt als angenommen (Melian et al. 1999; Wang et al. 1996). Diese Überdosis ist verursacht durch die hohe Zahl an Elektronen in dichteren Materialien wie v.a. Metallen (Farahani et al. 1990). Hier lösen sich die Sekundärelektronen von der Atomhülle des metallischen Elements, werden zurück geschlagen und bewirken so einen größeren Streueffekt (Melian et al. 1999; Wang et al. 1996). Dies hat zur Folge, dass die Mundhöhle und die Zähne von Kopf- und Halstumorpatienten, die mit zahnmedizinischen Materialien versorgt sind und eine Radiotherapie bekommen, stärkeren und höheren Dosen von Strahlung ausgesetzt sind. Die elektronische Rückstreuung von den zahnärztlichen Materialien kann dadurch auch noch stärker die umliegenden Weichgewebe schädigen (Farahani et al. 1990) und z.B. durch Verursachung einer Mukositis (Reitemeier et al. 2002; Mail et al. 2013) zum Erfordernis einer Einschränkung der den Tumorzellen geltenden Strahlentherapie, verbunden mit Verringerung der für die Tumorbekämpfung erforderlichen Strahlendosis, führen (Kaanders et al. 1992). Die Arbeitsgruppe um Farahani (Farahani et al. 1990) zeigte in ihrer Studie den Effekt der Absorption von Strahlen bei ausge- 
wählten zahnärztlichen Materialien wie Amalgam oder Nickel-Titan auf. Sie stellten fest, dass die Absorption weit weniger relevant ist als die rückwärtige Streustrahlung.

Schon einfache intraorale Schutzobjekte wie Gingivaretraktoren mit einer ausreichenden Dicke können, in dem sie die rückwärtigen Streustrahlen reduzieren, helfen zu vermeiden, dass unnötige Strahlung auf gesundes Gewebe gelangt und Weichgewebsschäden verursacht (Farahani et al. 1990; Kaanders et al. 1992; Mail et al. 2013; Reitemeier et al. 2002). Nach Reitemeier et al. (2002) können diese Rückstreueffekte an den Oberflächen von zahnärztlichen Materialien einen Anstieg der Dosis auf bis zu 170 \% im Vergleich mit der Bestrahlungsdosis ohne zahnärztliche Materialien verursachen. Je höher die atomische Zahl des Materials ist, desto höher war der Anstieg der Dosis. Die Ausdehnung der Rückstreueffekte betrug maximal $4 \mathrm{~mm}$.

Fazit der Studie von der Arbeitsgruppe um Reitemeier (Reitemeier et al. 2002) war, dass der herausgefundene beträchtliche Anstieg von $170 \%$ es verlangt, die Weichgewebe um die zahnärztlichen Materialien vor Strahlung zu schützen. Die Rückstreueffekte deuten darauf hin, dass das Weichgewebe effektiv mit einem $3 \mathrm{~mm}$ starken Weichgewebsretraktor abgeschirmt werden sollte (Chin et al. 2009; Farahani et al. 1993; Reitemeier et al. 2002).

In einer Studie von Chin et al. (2009), die die Rückstrahleffekte bei zahlreichen Konfigurationen von zahnmedizinischen Arbeiten in vitro untersuchte, wurde herausgefunden, dass festsitzender Zahnersatz aus Vollmetall die höchsten Anstiege der Strahlendosis verursacht. Währenddessen kommt es nach Chin et al. bei konservierenden Versorgungen wie z.B. Amalgam zu keinen signifikanten Dosiserhöhungen. Auch Restaurationen mit einem Keramikveneer haben nur eine geringe Erhöhung der Strahlendosis zur Folge.

Die Angaben zur lokalen Dosiserhöhung variieren jedoch abhängig vom verwendetem Werkstoff und Versuchsaufbau.

Gibbs et al. (1976) gaben die lokale Dosiszunahme an Amalgamfüllungen mit 109 \%-118\% an, während Thilmann und seine Arbeitsgruppe (Thilmann et al. 1995) sogar Dosiserhöhungen von 161 \% für Amalgam nachwiesen. 


\subsection{Auswirkung von Strahlentherapie auf die Zahnhartsubstanz}

Erstaunlicherweise ist wenig Genaues bekannt über die direkte Auswirkung von Strahlentherapie auf die Zahnhartsubstanzen (Andrews und Griffiths 2001a). Effekte wie die Demineralisationstiefen und die Löslichkeit des Schmelzes sind kontrovers diskutiert worden. Manche Studien können keinen Unterschied zwischen bestrahltem und unbestrahltem Schmelz (Shannon et al. 1978) und Dentin (Sperandio et al. 2001) deutlich machen und andere wiederum beschreiben eine erhöhte Löslichkeitsrate nach der Bestrahlung im Zahnschmelz verglichen mit unbestrahltem Zahnschmelz (Pioch et al. 1991). Grötz et al. (1998) wiesen nach, dass nach der Bestrahlung der Zahnschmelz „signifikante mikromorphometrische Unterschiede im Demineralisationverhalten" aufzeigt. Pioch et al. (1991) folgerten, da die „Schmelzqualität per se verändert wird“, dass dies im Zusammenhang stehen muss mit einer durch die ionisierenden Strahlen hervorgerufenen Veränderung in der organischen Matrix des Schmelzes. In einer aktuellen Studie wurde auch festgestellt, dass Bestrahlung die Mikrohärte und Mikromorphologie sowohl von Schmelz wie Dentin beeinflusst (Gonçalves et al. 2014).

Außerdem wurde gezeigt, dass die medizinische Radiotherapiedosis von 25 Gy keine Auswirkungen auf die Schmelzstärke oder seine Beständigkeit gegenüber Demineralisationen aufweist (Rodrigues et al. 2004).

So gilt generell, bei der Dosis, die bei einer Bestrahlung verwendet werden soll, Folgendes zu beachten: Bei einer Dosis unter 30 Gy ist der Schaden an den Zähnen bedeutend geringer, da hier die Speicheldrüsen noch nicht betroffen sind. Bei einer Dosis von 30-60 Gy ist der nachteilige Effekt der Dosis schon gravierender, da sie verbunden ist mit dem Beginn der Zerstörung der Speicheldrüsen. Eine kritische Stufe ist die Dosis >60 Gy, die vermutlich mit der direkten Einwirkung der Strahlung auf die Zahnsubstanz einhergeht. Aus diesen Werten ergaben sich Empfehlungen für einzuhaltende Grenzen bei der Planung (Walker et al. 2011).

Kielbassa et al. (2002) folgerten, dass die Demineralisationsprozesse im bestrahlten Dentin durch regelmäßige Anwendung eines Fluoridgels erschwert werden können.

Eine fortschreitende Störung der Schmelz- und Dentinmorphologie wurde mit steigender Strahlendosis beobachtet (de Siqueira Mellara et al. 2014). 


\subsection{Untersuchungsmethoden von Randspalten an Füllungsmaterialien}

\subsubsection{Klinisch mit Sonde und Spiegel}

Die Proben und Füllungsränder wurden nach klinischem Vorbild mit Spiegel und Sonde abgefahren. Diese Methode wird bei vielen großen klinischen Studien benutzt. Jedoch wird sie als sehr subjektiv und nur in geringem Maße standardisierbar gewertet.

\subsubsection{Lichtmikroskopie}

Bei dem in unserer Studie angewendeten Verfahren wird eine zweidimensionale Abbildung des untersuchten Objektes geschaffen. Die Vorteile des Verfahrens sind neben der einfachen Handhabung ohne großen apparativen Aufwand auch, dass dieselben Proben mehrfach untersucht werden können, da sie keinen alterierenden Behandlungen unterzogen werden.

Als Nachteile werden jedoch die geringe Tiefenschärfe, das Vorhandensein zusätzlicher Lichtquellen und die relativ geringe Vergrößerung diskutiert.

\subsubsection{Raster-Elektronen-Mikroskopie}

Mit dem Raster-Elektronen-Mikroskop ist es mit großem apparativem und vorzubereitendem Aufwand möglich, Bilder mit hoher Tiefenschärfe und guter räumlicher Darstellung anzufertigen. Um diese Bildaufnahme möglich zu machen, wird die zu Beginn in einem Sputter mit Kohle oder Metallen besputterte Probe durch einen gebündelten Primärelektronenstrahl zeilenförmig in einem auf die Probe gezogenen Hochvakuum abgefahren. Auf der Probe entstehen während dieses Prozesses Sekundärelektronen, welche das oberflächentopographische Bild ergeben.

In der quantitativen Randanalyse wird diese Methode heute häufig in Kombination mit der Replikatechnik angewandt. Die zu untersuchende Probe wird abgeformt und die Modelherstellung ist aus verschiedenen Materialien möglich. Sehr beliebt und als die beste Detailwiedergabe erreichend gelten Silikonabformmaterialien (z.B. Xantonpren Blau) (Ku- 
sy und Leinfelder 1977) in Kombination mit Epoxidharz als Modellmaterial (Replika)(Barnes 1978).

\subsection{Fragestellung}

Unsere Untersuchung will evaluieren, ob an der Grenzfläche zwischen Zahnhartsubstanz und den Füllungsmaterialien Amalgam und Komposit bei der für Tumorpatienten im KopfHals-Bereich genutzten Strahlengesamtdosis von 60 Gy oder einer experimentellen Überdosis von 90 Gy Veränderungen auftreten, die unter dem Auflichtmikroskop bei 66-facher Vergrößerung sichtbar werden. 


\section{Versuchsplan}

Es soll wie folgt vorgegangen werden:

Von ca. 30 frisch aus den Unterkiefern von Rindern extrahierten Zähnen werden die bukkalen und lingualen Flächen nach oben ausgerichtet und in dem Kaltpolymerisat Paladur ${ }^{\circledast}$ eingebettet. Die Verunreinigungen der Oberflächen werden mit Scalern mittlerer Größe (Hu-Friedy ${ }^{\circledR}$ ) beseitigt. Anschließend werden mit einem einheitlich fest gelegten Bohrgerät und eingespannten Diamantbohrern in Ausdehnung, Volumen und Tiefe definierte, gleiche Kavitäten in die Schmelzflächen gebohrt. 20 Kavitäten werden mit dem Füllungsmaterial Amalgam (Amalcap Plus ${ }^{\circledR}$ ) und 20 Kavitäten mit dem zahnfarbenen Füllungsmaterial Komposit (Venus A3) gefüllt.

Die Oberflächen und Grenzen zwischen den Füllungsmaterialien und dem Schmelz werden vor und nach der Bestrahlung in einer experimentellen Röntgenstrahlanlage unter einem Auflichtmikroskop mit 66-facher Vergrößerung auf Veränderungen beurteilt und um einen Vergleich herzustellen - fotografiert.

10 Füllungen aus Amalgam und 10 Füllungen aus Komposit werden mit einer Dosis von 60 Gy, die anderen 20 Füllungen (10 Amalgamfüllungen; 10 Kompositfüllungen) werden mit einer Dosis von 90 Gy bestrahlt.

Von allen 40 Füllungen und ihrer Grenzfläche Füllungsmaterial-Zahn werden vor und nach der Bestrahlung mit dem Mikroskop Zeiss Stemi SV11 und dem Moticam Images Plus Programm jeweils eine Aufnahme gemacht.

Diese Aufnahmen werden stets zwischen zwei Markierungsstrichen auf der Höhe zwischen zwei und drei Uhr angefertigt.

Zusätzlich werden alle 40 Füllungen einer klinischen Untersuchung unterzogen. An der Position der Füllungen zwischen sechs und sieben Uhr wird mit einer zahnärztlichen Sonde die Grenzfläche Füllung-Zahn auf eventuelle Unebenheiten und mögliche aufgetretene Spalten untersucht. 
Tabelle 1: Schematische Darstellung des Versuchsablaufes

Ca.30 aus Unterkiefern von Rindern extrahierte Zähne werden von Geweberesten und Verunreinigungen gereinigt

Bukkale und linguale Flächen werden nach oben im Kaltpolymerisat Paladur ausgerich-tet und eingebettet

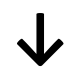

in Bohrmaschine eingespannte Diamantboh-rer werden in standardisiertem Bohrvorgang in definierter Tiefe versenkt

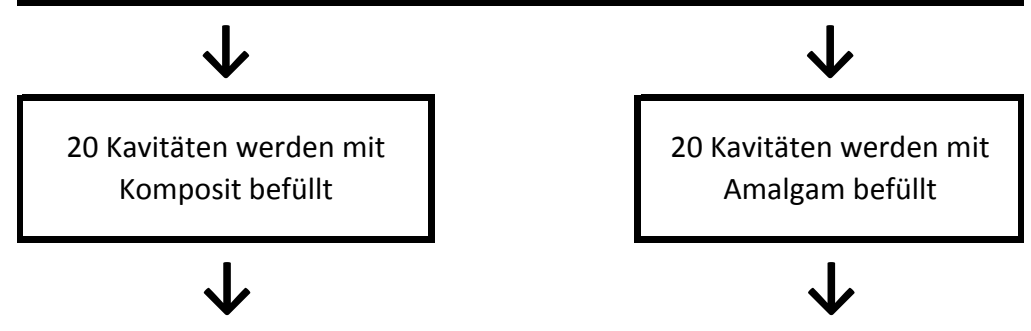

Vor Bestrahlung: Visuelle Beurteilung und Fotografie aller 40 Kavitäten und ihres Randspaltes an der Posi-tion zwischen zwei und drei Uhr mit Auflichtmikroskop bei 66-facher Vergrößerung;

Klinische Untersuchung mit Sonde der Grenzfläche Zahn-Füllung an der Position zwischen sechs und sie-ben Uhr

\begin{tabular}{|c|c|}
\hline $\begin{array}{c}\text { Bestrahlung von 10 Füllungen } \\
\text { mit Komposit und 10 } \\
\text { Füllungen mit Amalgam mit 60 } \\
\text { Gy }\end{array}$ & $\begin{array}{c}\text { Bestrahlung von } 10 \\
\text { Füllungen mit Kom-posit } \\
\text { und 10 Füllun-gen mit } \\
\text { Amalgam mit } 90 \text { Gy }\end{array}$ \\
\hline $\begin{array}{c}\text { Nach Bestrahlung: Visuelle Beurteilung und Fotografie aller 40 Kavitäten an } \\
\text { derselben Position mit dem Auflichtmikroskop; Bei der klinischen }\end{array}$ \\
\begin{tabular}{|l} 
Untersuchung wurde mit einer Sonde die Grenzfläche Zahn-Füllung an der \\
selben Position abgefahren
\end{tabular} \\
\hline
\end{tabular}




\section{Material und Methoden}

\subsection{Material}

\subsubsection{Rinderzähne}

Der Zahnschmelz von Rindern ähnelt in seiner Makro- und Mikromorphologie dem Zahnschmelz von Menschen (Oesterle et al. 1998; Schilke et al. 2000).

Aus diesem Grund stellen bovine Zähne mittlerweile eine gute und verbreitet genutzte Alternative zu humanen Zähnen in In-vitro-Untersuchungen dar (Yassen et al. 2011).

Im Vergleich zu Rinderzähnen zeigen Schweinezähne dagegen eine sehr unterschiedliche Konfiguration der Schmelzmorphologie auf (Reis et al. 2004).

Nach Oesterle und Mitarbeitern (Oesterle et al. 1998) weisen Rinderzähne eine größere und einheitlichere Oberfläche auf als menschliche Zähne. In den meisten Fällen sind bei ihnen keine kariösen Veränderungen auszumachen. Außerdem sind Rinderzähne günstig und in großen Mengen erhältlich. Auch sind auf ihnen nicht so häufig Schädigungen durch Umwelteinflüsse wie z.B. wechselnde Ernährungsgewohnheiten zu erkennen.

Auch der Kalziumgehalt wies nach Davidson et al. (1973) Ähnlichkeiten bei Rinder- und Menschenzähnen auf.

Neben der chemischen Zusammensetzung zeigen auch die mechanischen Eigenschaften wie Härte und Dichte eine gute Übereinstimmung mit menschlichen Zähnen (Davidson et al. 1973).

Dennoch ist anzumerken, dass der Zahnschmelz von Rinderzähnen etwas weicher und poröser ist als menschlicher Zahnschmelz. Auch der durchschnittliche Durchmesser der Schmelzkristalle und Dentintubuli ist bei Rinderzähnen im Vergleich größer (mit einem Verhältnis von Rind : Mensch von 1,6:1) (Arends und Jongebloed 1978; Schilke et al. 2000).

\subsubsection{Künstlicher Speichel}

Während der vorbereitenden Maßnahmen und im weiteren Versuchsverlauf wurden die Zähne in künstlicher Speichellösung, die alle sieben Tage erneuert wurde, gelagert. 
Die Herstellung des künstlichen Speichels erfolgte nach einer Rezeptur von Klimek et al. (1982):

Tabelle 2: Zusammensetzung des künstlichen Speichels

\begin{tabular}{|l|l|}
\hline Chemikalien & Anzahl in Gramm \\
\hline Ascorbinsäure $^{1}$ & 0,006 \\
\hline Glucose $^{1}$ & 0,090 \\
\hline Natriumchlorid $^{1}$ & 1,740 \\
\hline Kalziumchlorid $^{2}$ & 0,675 \\
\hline Ammoniumchlorid $^{1}$ & 0,480 \\
\hline Kalziumchlorid $^{1}$ & 3,810 \\
\hline Natriumrhodanid $^{3}$ & 0,480 \\
\hline Kalziumhydrogenphosphat $^{1}$ & 0,990 \\
\hline Harnstoff $^{1}$ & 0,600 \\
\hline Di-Natriumhydrogenphosphat $^{1}$ & 1,020 \\
\hline Aqua dest. $^{1}=$ Roth GmbH, Karlsruhe, D $^{2}=$ Merck KG, Darmstadt, D $_{3}=$ Fluka Chemie GmbH, Buchs, CH & 3 Liter \\
\hline
\end{tabular}

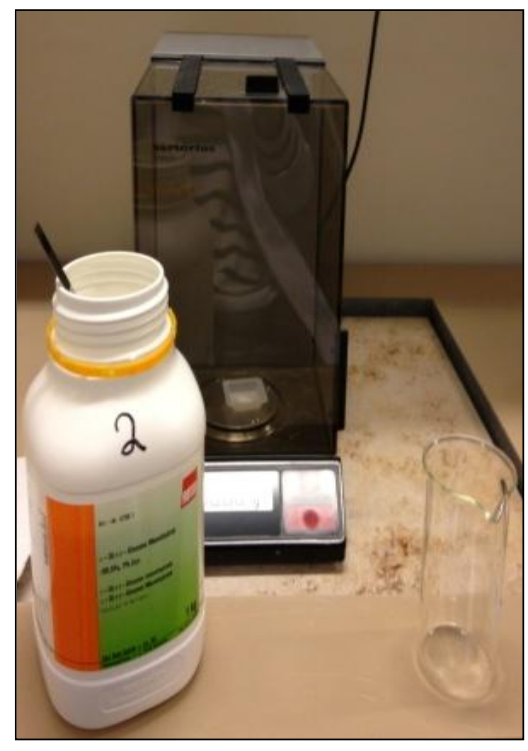

Abbildung 1: Speichelsubstanz und die Analysenwaage, mit der sie abgemessen wurde 
Die aufgeführten Substanzen wurden auf einer Analysenwaage abgewogen (Abb.1), danach vermischt und jeweils in einem mit destilliertem Wasser gereinigten Behälter mit 3 I Aqua dest aufgefüllt. Anschließend wurden sie mit einem Magnetrührer ca. vier Std verrührt, bis sich alle Bestandteile gelöst hatten.

\subsubsection{Verwendete Materialien und Geräte}

- $\quad$ Rinderzähne, Schlachterei Sebert's, Göttingen-Geismar, Deutschland

- $\quad$ Künstlicher Speichel, Substanzen, siehe Tabelle 2

- $\quad$ Analysenwaage Sartorius Research, Sartorius, Göttingen, Deutschland

- $\quad$ EXAKT Trennsystem Standard, Apparatebau, Norderstedt, Deutschland

- Gießform, Sonderanfertigung, wissenschaftliche Werkstatt des Universitätsklinikums Göttingen, Deutschland

- Schraubzwingen

- $\quad$ Paladur ${ }^{\circledR}$, Heraeus-Kulzer, Hanau, Deutschland

- $\quad$ Peripheriwachs, Sigma, Heraeus-Kulzer, Hanau, D

- Anmischbecher, Anrührspatel

- Bohrmaschine der Firma Metabo( T6 elektronic), Nürtingen, D

- $\quad$ Diamantbohrer, Komet, Lemgo, D

- $\quad$ Amalgamkapseln Amalcap Plus ${ }^{\circledR}$ von Ivoclar Vivadent dental, Ellwangen, D

- $\quad$ Amalgampistole, Hu-Friedy, Rotterdam, NL

- $\quad$ Kugelstopfer und Planstopfer, Hu-Friedy, Rotterdam, NL

- $\quad$ Amalgam-Rüttler Silamat ${ }^{\circledR}$, Ivoclar Vivandent dental, Ellwangen, D

- $\quad$ Greenie, Brownie, Arkansas, Komet, Lemgo, D

- $\quad$ Komposit Venus A3, Heraeus- Kulzer, Hanau, D (Exp.date 2016-06)

- $\quad$ Prime\&Bond Optibond ${ }^{\circledR} \mathrm{FL}, \mathrm{Kerr}$, Rastatt, D

- $\quad$ Micro Brush, 3M Espe, D

- $\quad 35 \%$ Phosphorsäure Ultra-Etch ${ }^{\circledR}$ Ultradent Products Inc., Utah, USA

- $\quad$ Lichthärtegerät, B.A. Optima 10, Hamburg, D

- $\quad$ Experimentelle Röntgenstrahlanlage der Firma Gulmay RS 225, Byfleet, Surrey, UK

- $\quad$ Auflichtmikroskop Zeiss Stemi SV11, Jena, D

- $\quad$ Moticam $23003.0 \mathrm{M}$ 
- $\quad$ Moticam Images Plus 2.0 ML

\subsubsection{Verwendete Füllungswerkstoffe}

Die Versuchsreihen wurden mit zwei verschiedenen Füllungswerkstoffen durchgeführt, die in der restaurativen Zahnmedizin häufig Anwendung finden.

Die chemische Zusammensetzung wurde den Herstellerangaben entnommen:

- Amalcap Plus ${ }^{\circledR}$ von Ivoclar Vivadent dental

○ Non-Gamma-2-freies, gamma-2-freies sphärisches Silberamalgam Zusammensetzung:

$\begin{array}{lc}\text { elementares Quecksilber } & 48,8 \text { Gew.-\% } \\ \text { elementares Silber } & 35,8 \text { Gew.-\% } \\ \text { elementares Zinn } & 9,3 \text { Gew.-\% } \\ \text { elementares Kupfer } & 6,1 \text { Gew.-\% }\end{array}$

- Komposit Venus A3 von Heraeus- Kulzer

○ Lichthärtendes Universalkomposit auf Feinstpartikel-Hybridbasis

\subsection{Methoden}

\subsubsection{Vorversuche}

4.2.1.1 Auswirkungen von Strahlentherapie auf Harvardzement, Füllungsmaterialien, Phantommetall, Nickel-Titan-Schlaufen

In den Vorversuchen wurde die Auswirkung von in der Strahlentherapie genutzten therapeutischen Dosen auf einzelne ausgewählte zahnärztliche Materialien untersucht und eventuelle Reaktionen dieser Materialien auf die Röntgenstrahlen (wie zum Beispiel Porositäten des Zementes, Erweichungen der Kunststoffe) beobachtet. Die einzelnen Proben aus allen Gruppen sind randomisiert zusammengestellt worden. Die erste Vorversuchsgruppe bestand aus sieben Objektträgern, die mit einer definierten Menge Zement bestrichen wurden. 
Die zweite Gruppe beinhaltete Phantommetallplättchen, von denen die eine Seite unbehandelt blieb und die andere Seite poliert wurde.

Eine dritte Gruppe setzte sich aus Phantommetallplättchen zusammen, auf denen NickelTitan-Schlaufen mit Harvard-Zement befestigt wurden.

Eine vierte Gruppe der Vorversuchsreihen bestand aus gleich hoch geformten Silikonblöcken mit Zähnen, in die Kavitäten gebohrt und Füllungsmaterialien appliziert wurden.

Von allen Gruppen wurden festgelegte Regionen, sowohl vor wie nach der Bestrahlung, mit dem Auflichtmikroskop AxioCam MRC Zeiss Lumar.V12 bei 12-facher Vergrößerung fotografiert. Eventuell auftretende Reaktionen der Materialien auf die Strahlen sollten auf den Fotos festgehalten werden können.

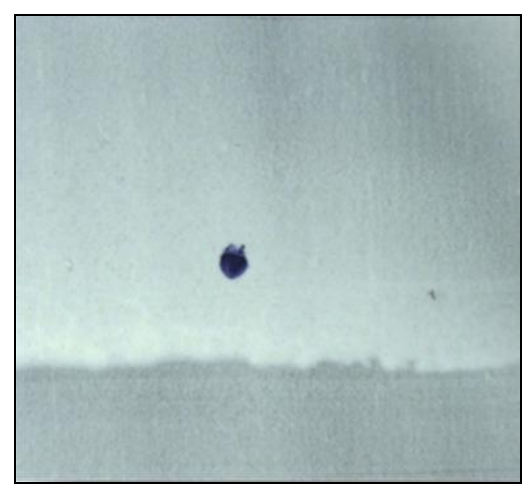

Abbildung 2: Vorversuche- Gruppe 1: Objektträger mit Harvardzement bestrichen: Bild vor Bestrahlung

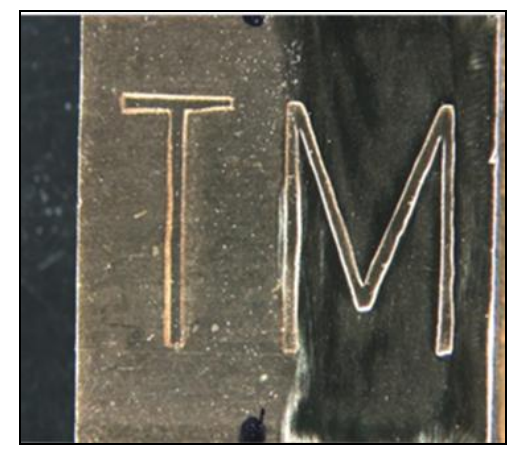

Abbildung 3: Vorversuche- Gruppe 2: Phantommetallplättchen Vergleich unbehandelte Seite: polierte Seite. Bild vor der Bestrahlung 


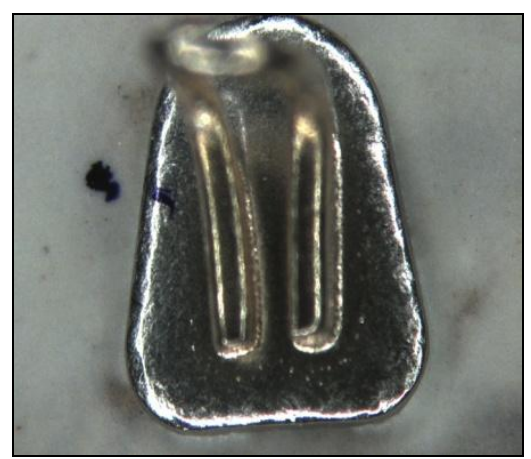

Abbildung 4: Vorversuche- Gruppe3: Nickel-Titan-Schlaufen mit Harvard-Zement auf Phantommetallplättchen befestigt. Bild vor der Bestrahlung

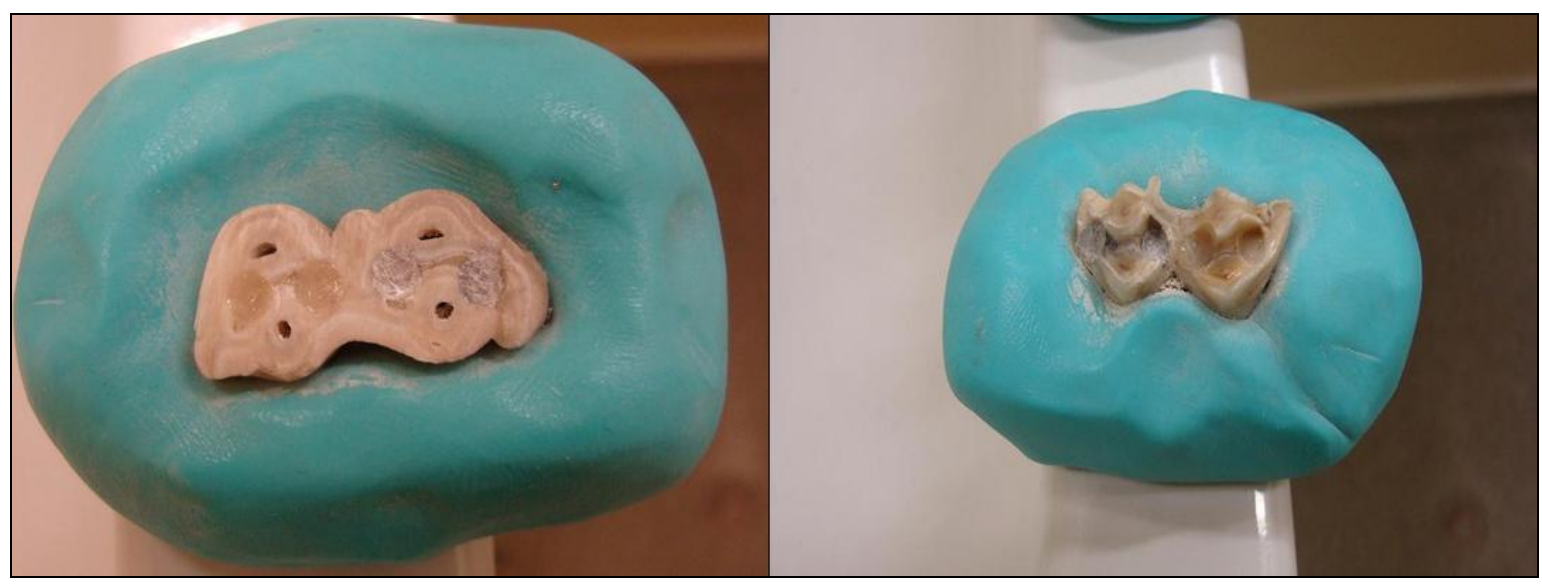

Abbildung 5: Vorversuche- Gruppe 4: Kavitäten mit Amalgam und Komposit in Rinderzähnen im Silikonblock

\subsubsection{Bestrahlung der Proben und Mikroskopie}

Die unterschiedlichen Reihen der Vorversuche sind in der experimentellen Röntgenstrahlanlage der Abteilung Strahlentherapie und Radioonkologie der Universitätsmedizin Göttingen mit einer Einzeldosis von 60 Gy für 12 Minuten bestrahlt worden.

Vor und nach der Bestrahlung wurden mit dem Auflichtmikroskop „AxioCam MRC Zeiss Lumar.V12“ in 12-facher Vergrößerung Fotos von ausgewählten Punkten angefertigt, um die Beschaffenheit des Materials vor und nach Bestrahlung vergleichen zu können.

\subsubsection{Versuch der Mikroskopie mittels des Raster-Elektronen-Mikroskops}

In Rinderzähne angelegte Kavitäten in Probeblöcken wurden mittels des RasterElektronen-Mikroskops (REM) untersucht. 
Zuerst sind die Zähne mit 70 \%-igem Alkohol gesäubert und anschließend für 24 Stunden in den Trockenschrank gelegt worden. Die Probeblöcke wurden mit dem kühlen Sputter SC 510 behandelt. Da keine Replikatechnik, sondern nur ein vereinfachtes Verfahren in den Vorversuchen angewandt wurde, brauchte sowohl der kühle Sputter wie auch das REM mehrere Stunden, um das benötigte Vakuum zu erlangen.

An den Grenzflächen Zahn-Füllung war deutlich zu erkennen, dass durch die für das REM notwendigen Verfahren wie Säuberung, Trocknung und Sputtern die Schmelzoberflächen und Grenzflächen schon vor der Bestrahlung geschädigt wurden. Aus diesem Grund nahmen wir Abstand von dem zur Verfügung stehenden REM und entschieden uns für das Auflichtmikroskop.

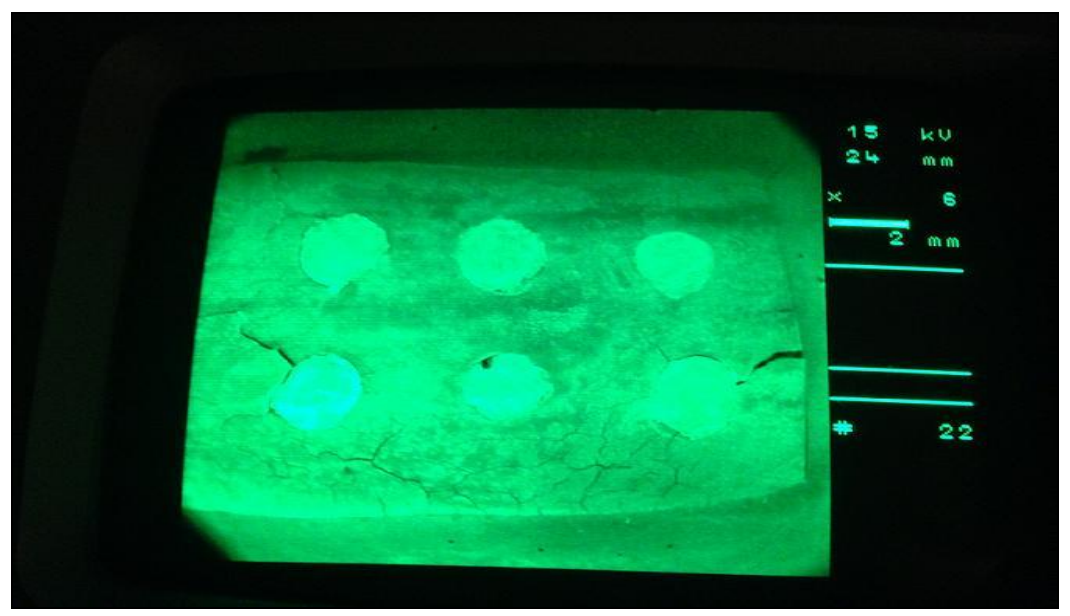

Abbildung 6: Probeblock mit Schmelzrissen im Raster-Elektronen-Mikroskop

\subsubsection{Hauptversuche}

\subsubsection{Herstellung und Vorbereitung der Rinderzahnproben}

Die Rinderzähne stammten von der Schlachterei Sebert's in Göttingen-Geismar. Das Alter der Tiere lag zwischen drei und fünf Jahren.

Nachdem die Tiere geschlachtet wurden, sind die Gewebereste von den Zähnen anschließend im Labor mit Skalpellen und scharfen Löffeln entfernt und die Schmelzoberflächen mit Scalern mittlerer Größe von Verfärbungen gereinigt worden.

Anschließend lagerten die Rinderzähne in künstlichem Speichel bei $7,5^{\circ} \mathrm{C}$ im Kühlschrank. Die Wurzeln wurden mit dem EXAKT Trennsystem Standard im Labor der Mund-KieferGesichtschirurgie unter Wasserkühlung abgetrennt. Ebenfalls wurden plane Flächen der 
Zähne als optimale Kavitätenflächen ausgewählt und die Zähne unter Wasserkühlung in eine passende Form gesägt. So war es möglich, sie in eine angefertigte Form mit Kunststoff einbetten zu können.

Die Kronen wurden gründlich auf kariöse Läsionen und Schmelzdefekte untersucht. Die Rinderzähne, die derartige Auffälligkeiten zeigten, wurden aussortiert.

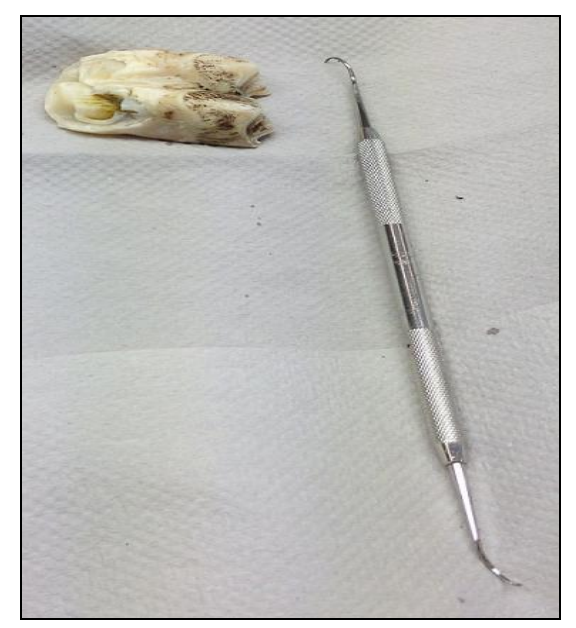

Abbildung 7: Säuberung der Zähne von Verfärbungen mittels eines Scalers

\subsubsection{Einbettung der Rinderzähne mit Paladur in einer Gießform}

In einer Gießform der Größe 20x50 mm, einer Sonderanfertigung der wissenschaftlichen Werkstatt der UMG, wurden die Zähne mit Sigma Peripheriwachs mittig platziert. Die Seiten der Gießform wurden mit Vaseline ausgestrichen und zwei der vier Seiten der Gießform mittels einer Schraubzwinge adaptiert.

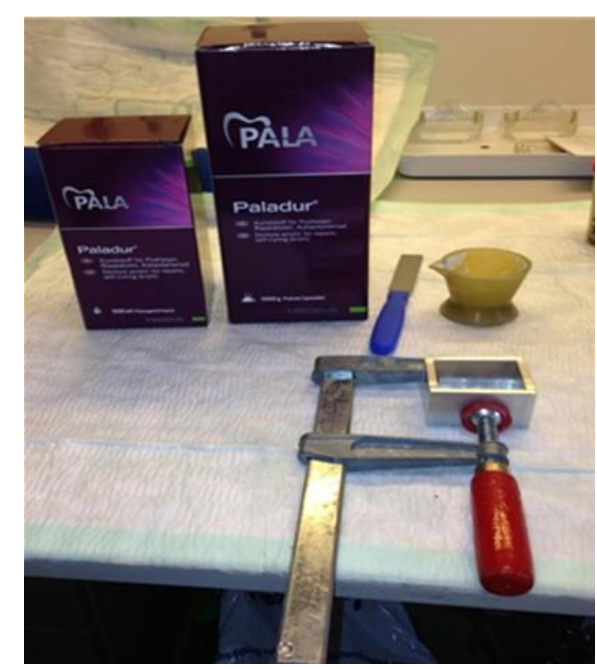

Abbildung 8: Kunststoff Paladur ${ }^{\circledR}$, Anrührbecher, Gießform und Schraubzwinge 
In einem Silikonanmischbecher wurden $13 \mathrm{~g}$ Pulver und 7,2 $\mathrm{ml}$ Flüssigkeit Paladur ${ }^{\circledR}$ mit einem Kunststoffanrührspatel verrührt. Sobald die Konsistenz eine leichte Zähflüssigkeit erreicht hat, wurde die genau für einen Block benötigte Kunststoffmasse langsam in die Gießform bis zum Rand eingefüllt. Die Zähne waren nun gleichmäßig von Kunststoff umgeben, und die Schmelzoberfläche ragte ebenmäßig aus dem Kunststoff heraus.

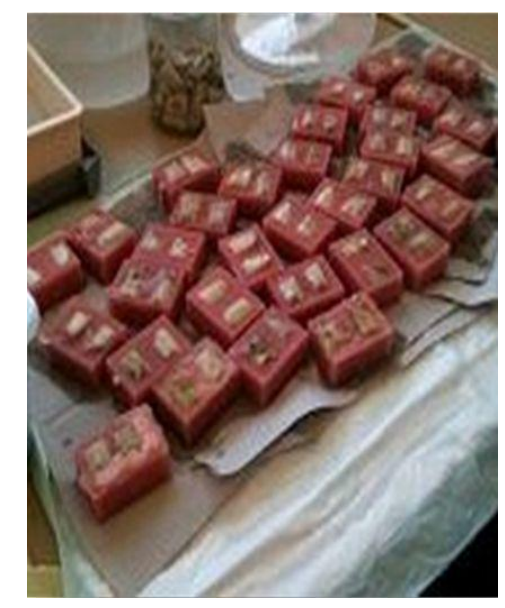

Abbildung 9: Rinderzähne eingebettet in Kunststoffblöcke

\subsubsection{Kavitätenpräparation und Applikation der Füllungsmaterialien Amalgam und}

\section{Komposit}

Auf den in Kunststoff eingebetteten Zähnen wurden in einem Abstand von 5 mm Punkte für die Bohrlöcher skizziert.

Die Kunststoffblöcke, die alle $25 \mathrm{~mm}$ breit und $20 \mathrm{~mm}$ hoch waren, wurden randomisiert in vier Gruppen eingeteilt.

Die Gruppe 1 bestand aus einem Kunststoffblock $A_{1}$ mit zwei Rinderzähnen, in die 10 Kavitäten angelegt wurden, die mit Amalgam gefüllt und einer Einfachdosis von 60 Gy bestrahlt werden sollten.

Die Gruppe 2 bestand aus Block $B_{1}$ und $B_{2}$ mit 10 Kavitäten, die mit Amalgam gefüllt und einer Überdosis von $90 \mathrm{~Gy}$ bestrahlt werden sollten. Aus herstellungstechnischen Gründen wurden hier wie bei Gruppe 3 und 4 die Kavitäten auf zwei Kunststoffblöcke verteilt.

Die Gruppe 3 bestand aus zwei Blöcken mit insgesamt 10 Kavitäten, die mit Komposit gefüllt und einer Einfachdosis von $60 \mathrm{~Gy}$ bestrahlt werden sollten.

Die Gruppe 4 bestand aus zwei Blöcken mit insgesamt 10 Kavitäten, die mit Komposit gefüllt und einer Überdosis von 90 Gy bestrahlt werden sollten (siehe Tabelle 3). 
Tabelle 3: Zuordnung der Füllungsmaterialien und unterschiedlichen Bestrahlungsdosen in vier Gruppen

\begin{tabular}{|l|l|l|l|l|}
\hline Gruppe & Füllungsmaterial & Kunststoffblock & Kavitätenanzahl & $\begin{array}{l}\text { Bestrahlungsdosis in } \\
\text { Gray }\end{array}$ \\
\hline $\mathbf{1}$ & Amalgam & Block $\mathrm{A}_{1}$ & 10 & 60 \\
\hline $\mathbf{2}$ & Amalgam & BlockB1+B2 & 10 & 90 \\
\hline $\mathbf{3}$ & Kunststoff & Block $\mathrm{B}_{1}+\mathrm{C}_{2}$ & 10 & 60 \\
\hline $\mathbf{4}$ & Kunststoff & BlockD $1+\mathrm{D}_{2}$ & 10 & 90 \\
\hline
\end{tabular}

Die Kavitäten der einzelnen Gruppen wurden randomisiert angelegt.

Die für das jeweilige Füllungsmaterial empfohlenen Diamantbohrer sind nacheinander in die Bohröffnung der Bohrmaschine der Firma Metabo (T6 Elektronik) eingespannt worden. Per Hebel konnten die Diamantbohrer gezielt 0,5 mm im Zahn versenkt werden. Um bei jeder Füllung die gleiche Tiefe zu garantieren, wurden die Diamantbohrer mit einer Markierung in Höhe von $5 \mathrm{~mm}$ zirkulär versehen. Bis zu dieser Markierung wurden die eingespannten Diamantbohrer versenkt.

Bei den Kavitäten für die Amalgamfüllungen (AgF) ist zuerst der Diamantbohrer von der Firma Komet "Zylinder kurz Größe 021" und anschließend der Diamantbohrer

von Komet „umgekehrter Kegel, lang Größe 016“ eingespannt worden. Diese genannten Bohrer sollten die für das Füllungsmaterial Amalgam vorgesehenen Unterschnitte in der Kavität ermöglichen und die Übergänge zwischen Kavitätenwand- und boden abrunden, um das Auftreten von Spannungen im Füllungsmaterial in diesem Bereich zu erschweren.

Für die Anlegung der Kavitäten für das Material Komposit wurden ebenfalls zwei unterschiedliche Diamantbohrer verwendet.

Um die Anschrägung der Schmelzprismen zu gewährleisten, wurde ein Diamantbohrer von Komet "Kugel rund Größe 010" eingesetzt, nachdem die Kavität mit dem Diamantbohrer von Komet "Zylinder kurz Größe 021" angelegt worden war.

Alle Kavitäten sind mit ausreichend Wasserkühlung und neuen Bohrern angefertigt worden. 


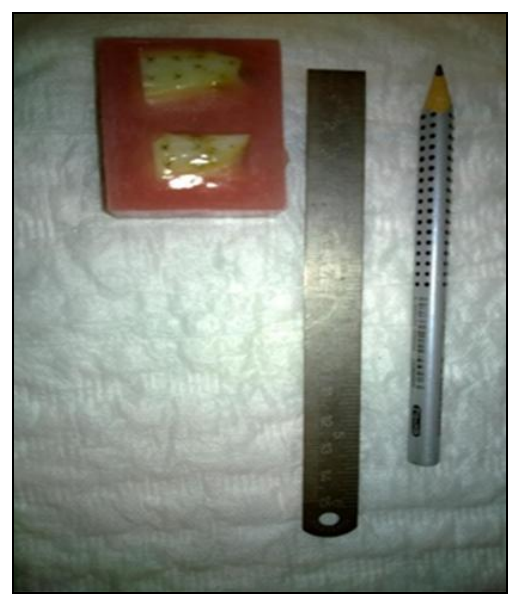

Abbildung 10: Markierung der Bohrlöcher im Abstand von 5 mm

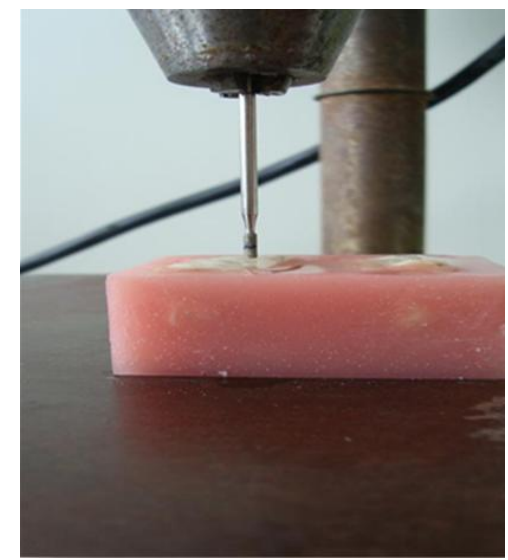

Abbildung 11: Block unter der Bohrmaschine Metabo liegend, Markierung des Diamantbohrers in Höhe von $5 \mathrm{~mm}$ zu erkennen

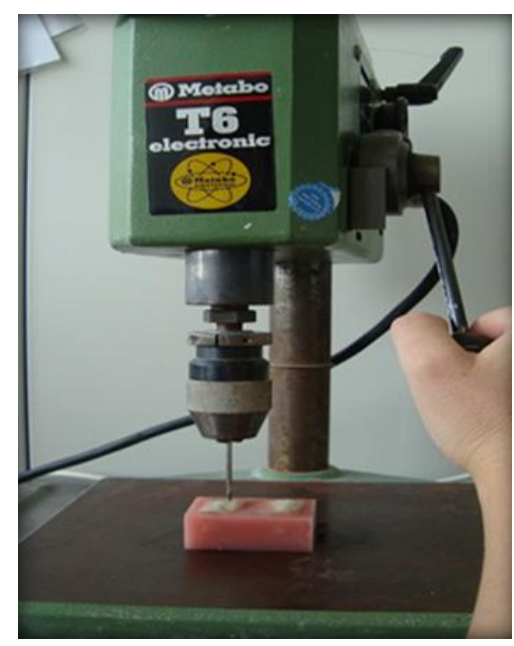

Abbildung 12 Betätigung des Hebels und standardisierter Bohrvorgang 


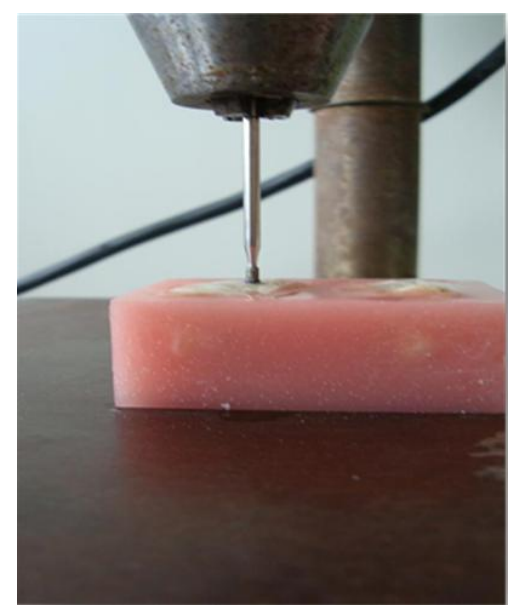

Abbildung 13: Gezielte Versenkung des Diamantbohrers bis zu der Markierung

Nach Anlegung, gründlicher Säuberung und Trocknung der Kavitäten wurden die Füllungsmaterialien in die Kavitäten appliziert.

Bei den Kompositfüllungen (KoF) ist zu Beginn die 35-\%ige Phosphorsäure Ultraetch ${ }^{\circledR}$, Ultradent Products Inc aufgetragen, 15 Sekunden auf dem Dentin und 30 Sekunden auf dem Schmelz belassen und anschließend 30 Sekunden mit Wasser abgesprüht worden. Anschließend folgte die Applikation des Adhäsivsystems Prime\&Bond von Optibond ${ }^{\circledR}$ FL. Der Primer wurde 15 Sekunden mit einem Einmal-Microbrush in die Hartsubstanz einmassiert und anschließend leicht verblasen.

Das sich anschließende Bonding wurde 15 Sekunden aufgetragen und 20 Sekunden lang ausgehärtet.

Die Applikation des Komposits Venus A3 dauerte mit der Inkrementtechnik pro Kavität 2 Minuten und 30 Sekunden. Die Dauer der Zwischenhärtungen betrug 20 Sekunden und die der Endhärtung 30 Sekunden. Ein Planstopfer und Heidemannspatel von HU-Friedy wurden als Applikations- und Stopfhilfe genutzt.

Mit einem Brownie und Greenie von Komet sind mit 3200 Umdrehungen/Minute für jeweils 10 Sekunden die applizierten Füllungen noch einmal poliert worden.

Mit der zahnärztlichen Sonde wurden die Ränder auf Überschüsse oder Spalten untersucht. 


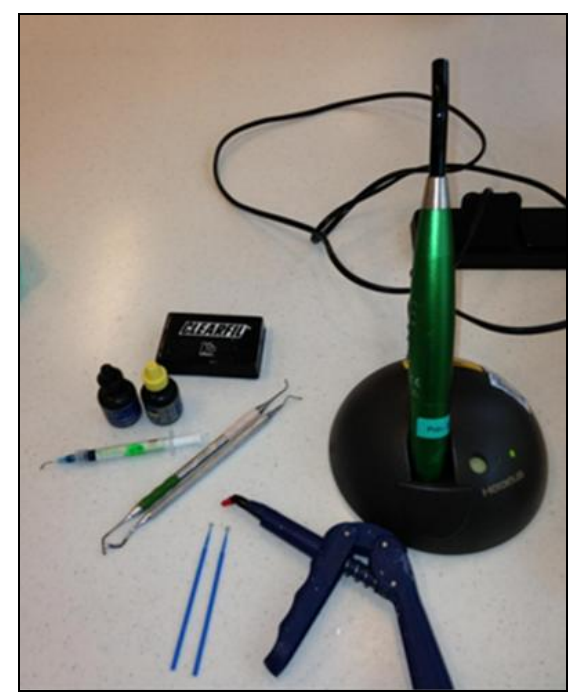

Abbildung 14: Materialien zur Applizierung der Kompositfüllungen

Bei den Amalgamfüllungen sind die Amalgamkapseln Amalcap Plus ${ }^{\circledR}$ von Ivoclar vivadent zum Einsatz gekommen. Amalcap Plus ${ }^{\circledR}$ ist ein Gamma-2-Phasen-freies Amalgam.

Nach der vom Hersteller vorgeschriebenen Mischzeit im Silamat ${ }^{\circledR}$ von 10 Sekunden wurde das plastische Material portionsweise mit einer Amalgampistole in die Kavität eingebracht. Zwei Kavitäten konnten mit einer Kapsel in der vom Hersteller vorgeschriebenen Zeit von 3-4 Minuten gefüllt werden. Kondensiert und modelliert wurde die applizierte Füllung mit einem Kugel- und Planstopfer.

Die Oberflächen der Amalgamfüllungen sind mit einem Kugelstopfer für 20 Sekunden/Kavität brüniert worden.

Die endgültige Politur mit Brownie und Greenie fand, ebenfalls wie vom Hersteller empfohlen, 24 Stunden nach Applizierung der Füllungen mit 3200 Umdrehungen/Minute bei ständiger und ausreichender Spraykühlung und Absaugung statt. 


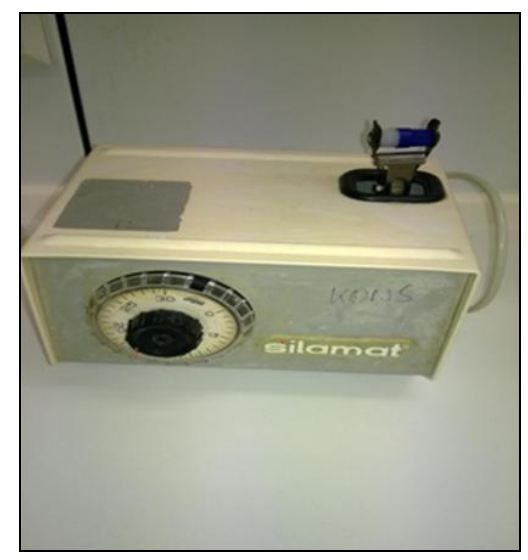

Abbildung 15: Amalgamtriturator Silamat ${ }^{\circledR}$ mit Amalgamkapsel Amalcap Plus ${ }^{\circledR}$ zur Applizierung der Amalgamfüllungen

\subsubsection{Bestrahlung}

Die Proben wurden je nach Material und Dosis wieder ihren vier Gruppen zugeteilt. Jede Gruppe wurde, wie oben beschrieben, je nach Material und Dosis in der experimentellen Röntgenstrahlanlage der Firma Gulmay RS 225 auf dem inneren Ring des Tellers platziert. Die Tischhöhe betrug $205 \mathrm{~mm}$. Die Tischhöhe beschreibt den Abstand von der Röntgenquelle zu dem bestrahlenden Objekt.

Die Röhrenspannung betrug 200 kV, die Stromstärke 15 mA und der Kupferfilter 0,5 mm. Bei Gruppe 1 und 3, den Blöcken mit Amalgam- und Kompositfüllungen, wurde nacheinander die Dosis 5 Gy pro Minute eingestellt, sodass die Proben insgesamt 12 Minuten und einer Einzeldosisstrahlung von $60 \mathrm{~Gy}$ ausgesetzt wurden.

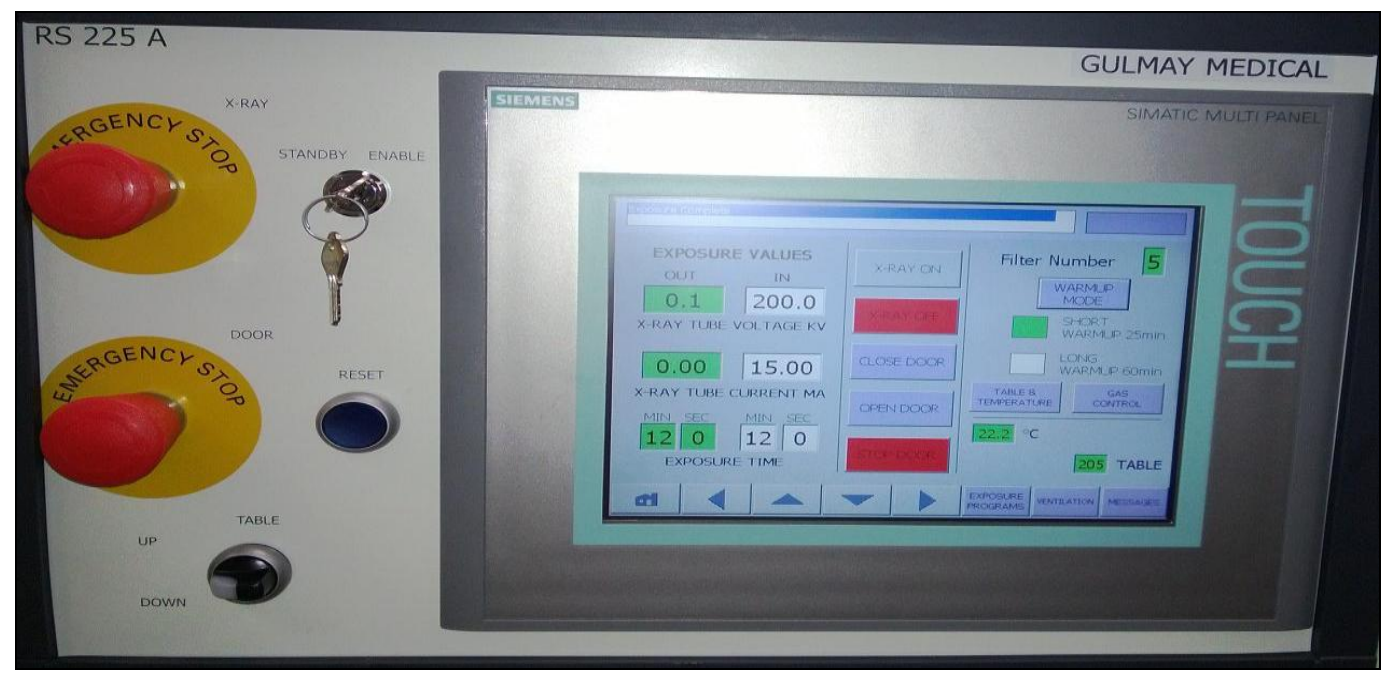

Abbildung 16: Bildschirm der experimentellen Röntgenstrahlanlage mit Bestrahlungszeit, Röhrenspannung, Stromstärke, Filter und Tischhöhe 
Dasselbe Verfahren wurde mit einer experimentellen Überdosis von $90 \mathrm{~Gy}$ angewendet. Die Blöcke aus Gruppe 2 und 4 mit jeweils zehn Amalgam- und Kompositfüllungen wurden nacheinander in die Röntgenstrahlanlage gelegt und einer Strahlendosis von 90 Gy ausgesetzt.

Diese setzte sich aus zwei Bestrahlungszeiten von 2x9 Minuten zusammen, mit dazwischen liegenden 15 Minuten Pause, um die Röntgenröhre abkühlen zu lassen.

Beide Blöcke des jeweiligen Füllungsmaterials fanden auf dem inneren Ring des Tellers in der Röntgenstrahlanlage Platz und die Tischhöhe betrug 205 mm.

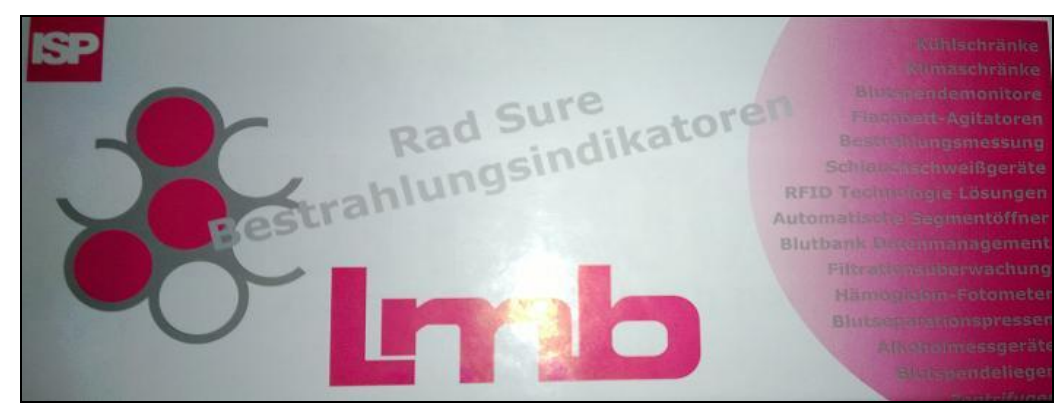

Abbildung 17: Bestrahlungsindikator zur Anwendung in der Strahlenkammer

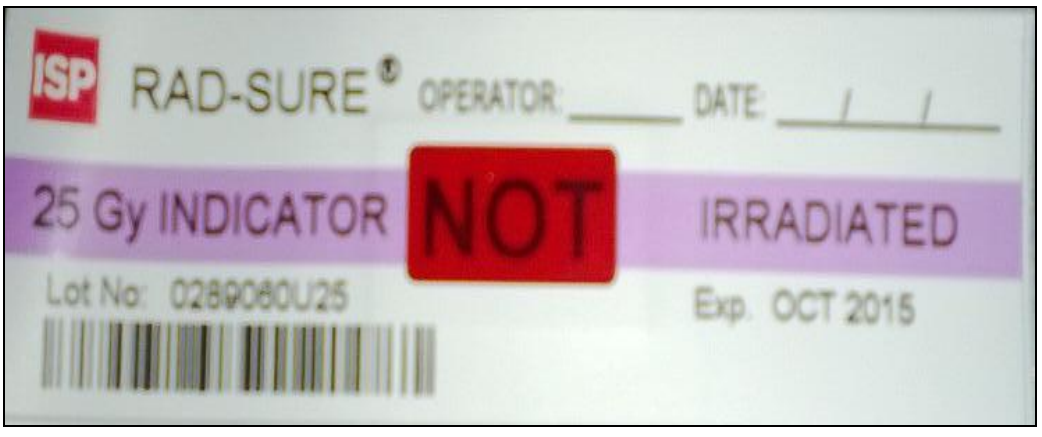

Abbildung 18: Unbestrahlter Bestrahlungsindikator

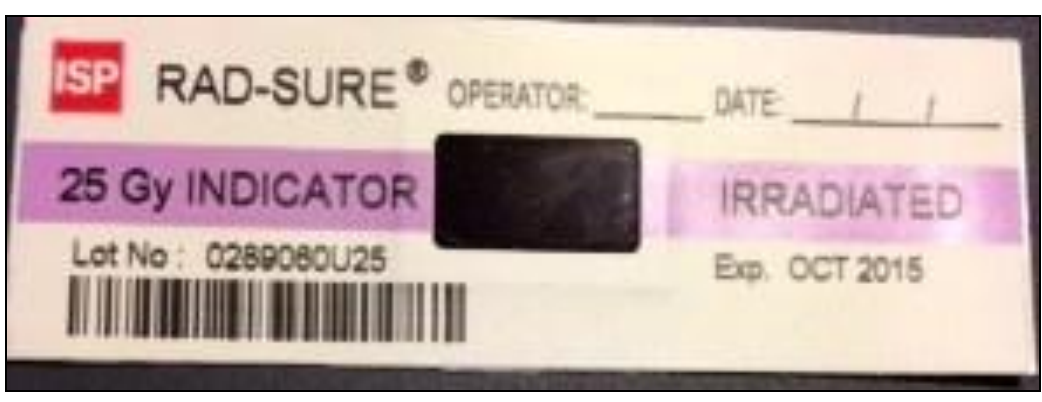

Abbildung 19: Bestrahlter Bestrahlungsindikator nach Bestrahlung unserer Proben mit 60 Gy 


\subsubsection{Klinische Sondierung und Vergrößerung}

Mittels einer zahnärztlichen Sonde wurde jede Füllung vor und nach der Bestrahlung in der Röntgenstrahlanlage untersucht, um eventuell durch die Strahlung aufgetretene Veränderungen festzustellen.

Die klinische Untersuchung wurde dieses Mal an der Grenzfläche Füllung-Zahn zwischen der sechs und sieben Uhr-Position durchgeführt, um die unter dem Mikroskop zu untersuchende Stelle zwischen zwei und drei Uhr nicht vor der Bestrahlung verändert und behandelt zu haben.

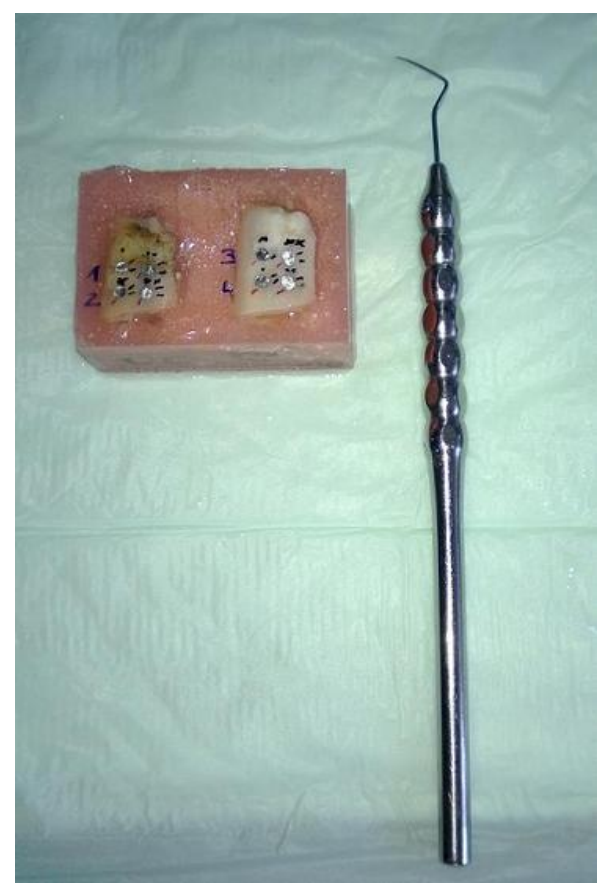

Abbildung 20: Klinische Untersuchung mit zahnärztlicher Sonde 


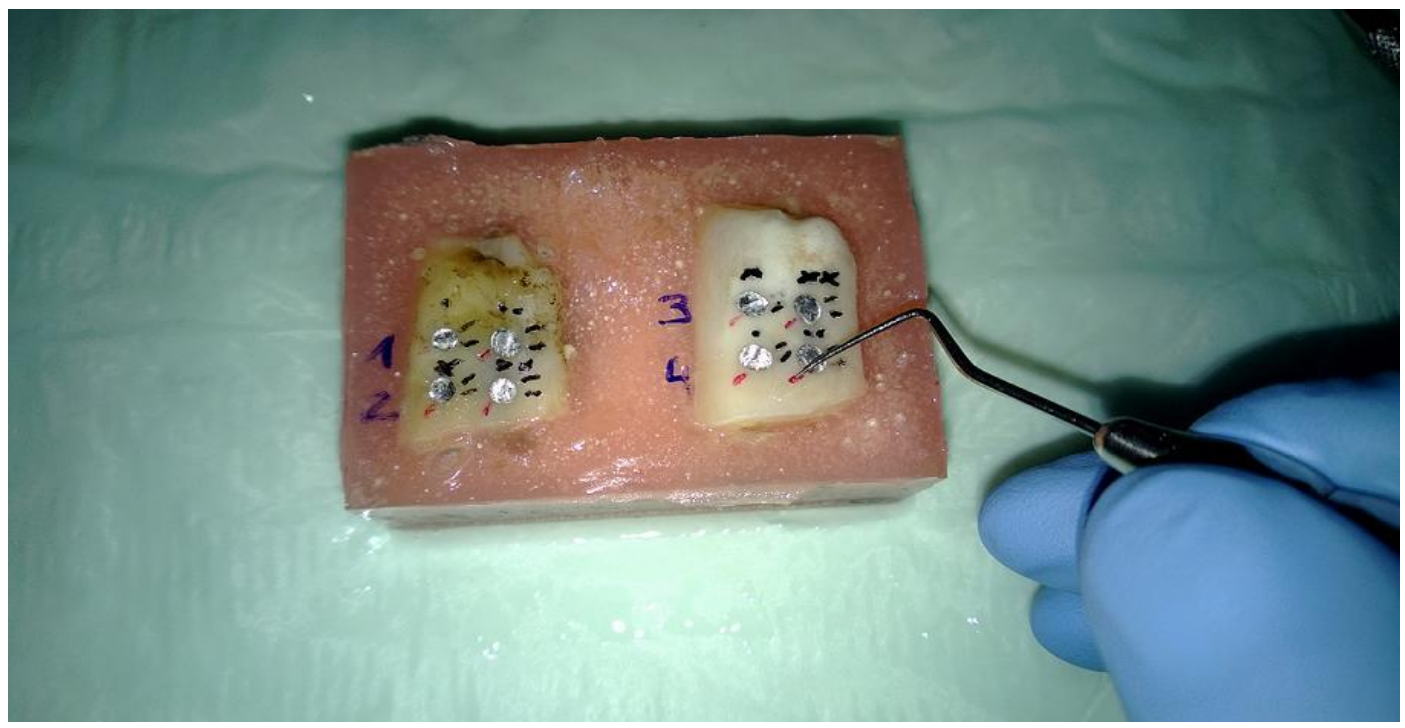

Abbildung 21: Probe mit zahnärztlicher Sonde am zu untersuchenden markierten Abschnitt

Bei Anwendung dieser Methode sollte nach definierten Kriterien, wie denen von Cvar und Ryge beschriebenen (Cvar und Ryge 2005) vorgegangen werden. Die Kriterien wurden entwickelt, um die ästhetischen Qualitäten und die funktionelle Leistung von zahnärztlichen Restaurationen widerzuspiegeln. Mit Noten wurden die einzelnen Kriterien (anatomische Form, Farbauswahl, Randverfärbung, Randspalt, Karies) bewertet. Die Bewertun-

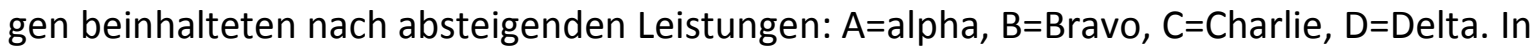
unserer Studie wurde nur das eine Kriterium "Randspalt" genutzt.

\subsubsection{Mikroskopie}

Mit dem Auflichtmikroskop Zeiss Stemi SV11 aus der Mund-Kiefer-Gesichtschirurgie der UMG sollten die Grenzflächen zwischen Zahn und Füllungsmaterial betrachtet werden.

Die am Mikroskop befestigte Kamera Moticam 2300 3.0 M Pixel USB 2.0 mit dem Kaltlicht Novoflex übertrug das Bild auf den Bildschirm. Mit dem Programm der Kamera, Motic Images Plus 2.0 ML, war es möglich, die Bilder aufzunehmen und ggf. aufgetretene Veränderungen an den Füllungsrändern, z.B. in Form von Spalten, zu vermessen.

Von dem markierten Bereich zwischen zwei und drei Uhr sind vor und nach den Bestrahlungen in der Röntgenstrahlanlage Bilder angefertigt worden. 


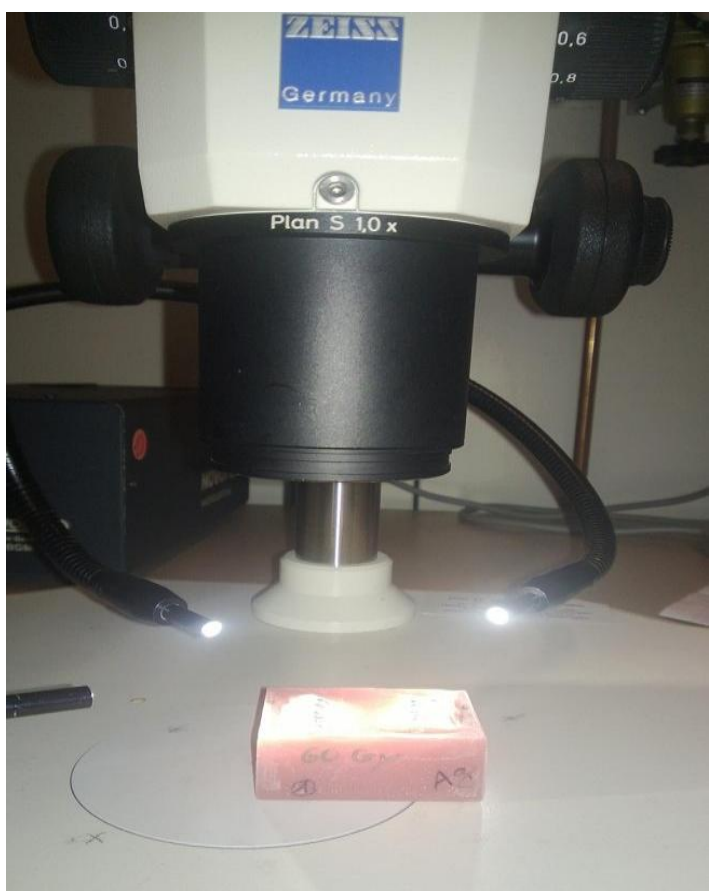

Abbildung 22: Auflichtmikroskop Zeiss Stemi SV11 mit Probe 


\section{Ergebnisse}

In der vorliegenden Arbeit sollte der Randschluss von den zwei Füllungsmaterialien Amalgam und Komposit zur Zahnhartsubstanz vor und nach Bestrahlung in der Röntgenstrahlanlage mit einer Dosis von 60 Gy und 90 Gy unter dem Auflichtmikroskop bei 66-facher Vergrößerung untersucht werden. Hierzu wurden randomisiert identische Kavitäten angelegt und die Füllungsmaterialien in einem reproduzierbaren standardisierten Verfahren appliziert.

\subsection{Ergebnisse der Vorversuche}

Zu Beginn sind in vereinfachten Vorversuchen ausgewählte zahnärztliche Materialien wie Harvardzement, Füllungsmaterialien, Phantommetall und Nickel-Titan-Schlaufen auf Veränderungen bei der Bestrahlung untersucht worden. Ausgewählte Ausschnitte sollten vor sowie nach Bestrahlung unter dem Auflichtmikroskop betrachtet und auf eventuelle Reaktionen dieser Materialien auf die Röntgenstrahlen untersucht werden.

Es ergaben sich anhand der mikroskopischen Darstellungen keine Veränderungen an den untersuchten Materialien, die mittels 6-facher Vergrößerung darstellbar waren.

Abbildung 23: Objektträger mit Zement vor und nach Bestrahlung

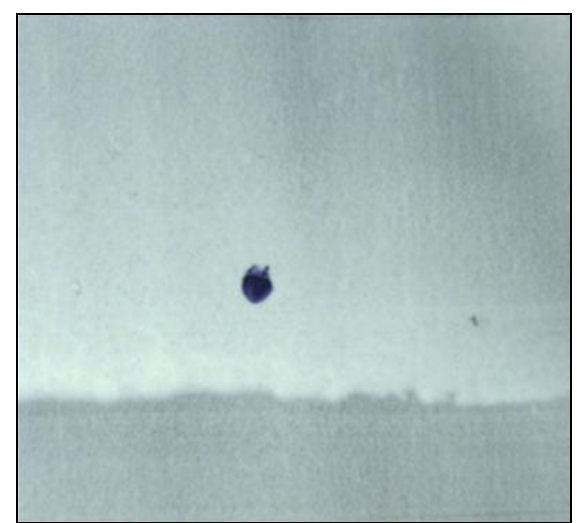

Abb.23: Objektträger mit Zement vor Bestrahlung

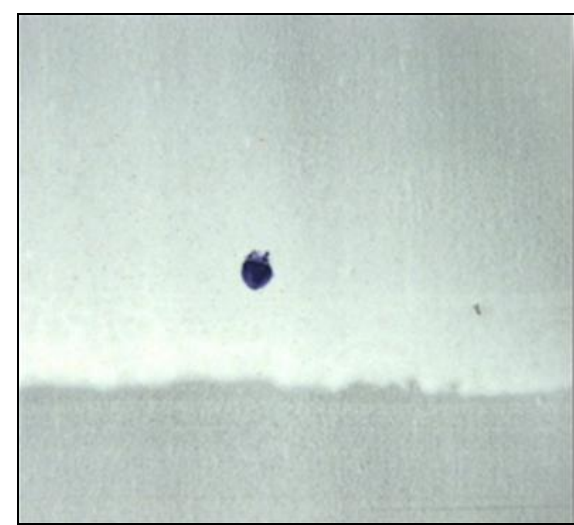

Abb. 23a: Objektträger mit Zement nach B. 
Abbildung 24: Phantommetallplättchen unbehandelte/polierte Seite vor und nach Bestrahlung

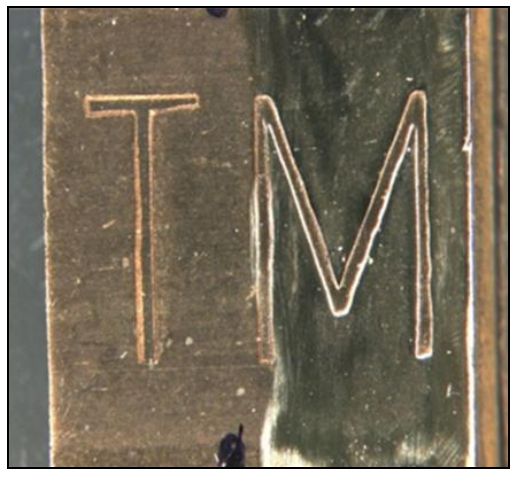

Abb.24: Phantommetallplättchen

Unbehandelte/polierte Seite, vor Bestrahlung

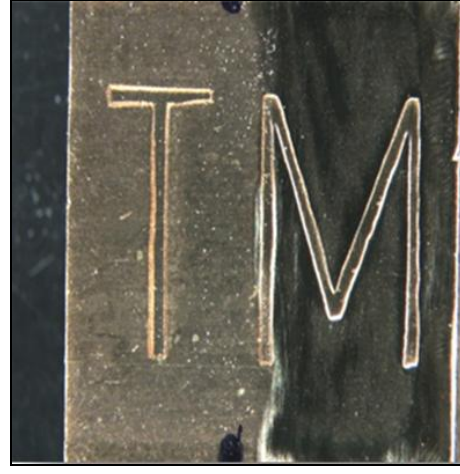

Abb.24a: Phantommetallplättchen unbehandelte/polierte Seite, nach Bestrahlung

Abbildung 25: Nickel-Titan-Schlaufe auf Zement befestigt vor und nach Bestrahlung

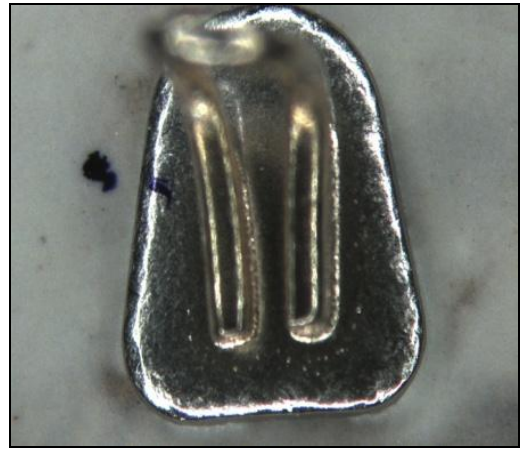

Abb.25: Nickel-Titan-Schlaufe auf Zement befestigt mit Markierung vor Bestrahlung

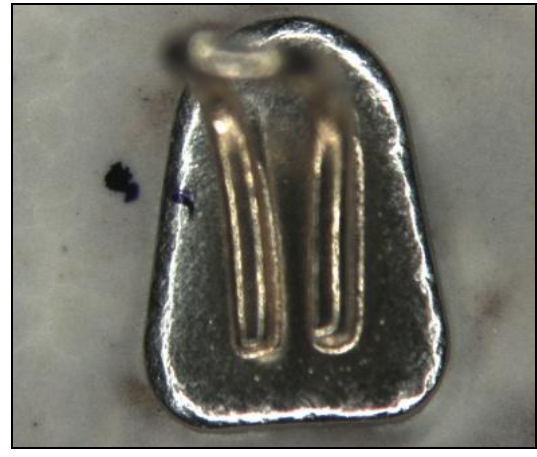

Abb.25a: Nickel-Titan-Schlaufe auf Zement befestigt mit Markierung, nach Bestrahlung

Abbildung 26: Amalgamfüllung (AgF) in Rinderzahn vor und nach Bestrahlung

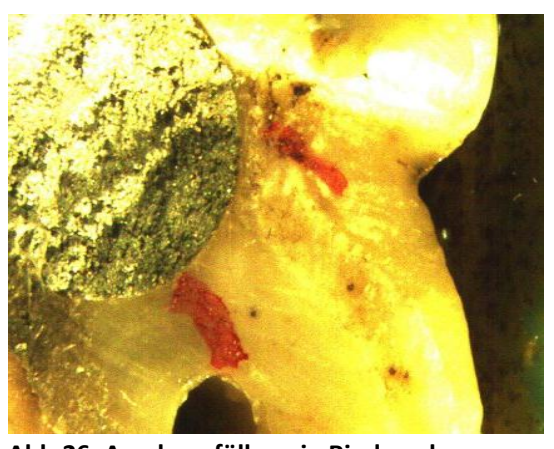

Abb.26: Amalgamfüllung in Rinderzahn

im Kunststoffblock vor Bestrahlung

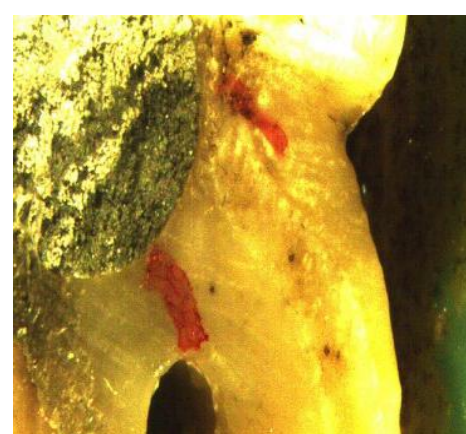

Abb.26a: AgF in Rinderzahn nach Bestrahlung 
Abbildung 27: Kompositfüllung in Rinderzahn vor und nach Bestrahlung

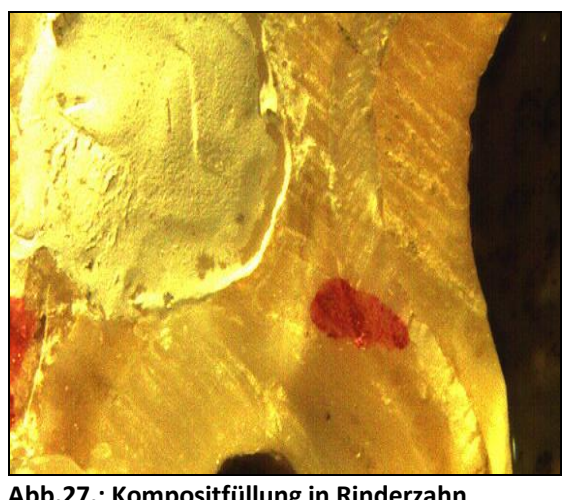

Abb.27.: Kompositfüllung in Rinderzahn vor Bestrahlung

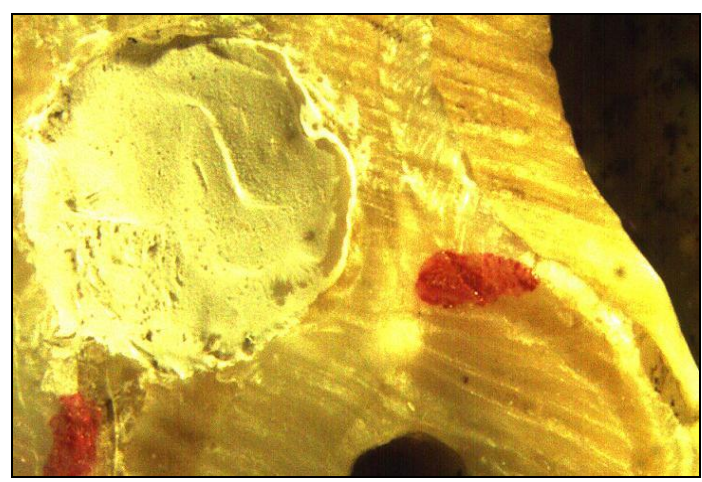

Abb.27a: Kompositfüllung nach Bestrahlung

\subsection{Ergebnisse der Hauptversuche}

\subsubsection{Ergebnisse der Untersuchungen unter dem Auflichtmikroskop}

Bei 66-facher Vergrößerung war mit dem Auflichtmikroskop Zeiss Stemi SV11 in 39 von 40 Fällen der untersuchten Proben keine signifikante Veränderung an der Grenzfläche ZahnFüllungsmaterial zu erkennen. Nach Aufnahme und Vermessung der Bilder mit dem Programm Motic Images Plus 2.0 ML konnte nur an einer untersuchten Füllung ein Spalt evaluiert und vermessen werden. Mit dem in dieser Studie verwendeten Randspaltuntersuchungsverfahren konnte somit weder statistisch noch auf den mit dem Auflichtmikroskop bei 66-facher Vergrößerung angefertigten Aufnahmen ein Unterschied zwischen dem Zustand der Füllungen vor und nach der Bestrahlung fest gestellt werden.

An einer Amalgamfüllung aus der Gruppe 1 konnte ein Spalt vermessen werden: 
Abbildung 28: Gruppe 1 Amalgamfüllungen (AgF) mit 60 Gray bestrahlt vor und nach Bestrahlung

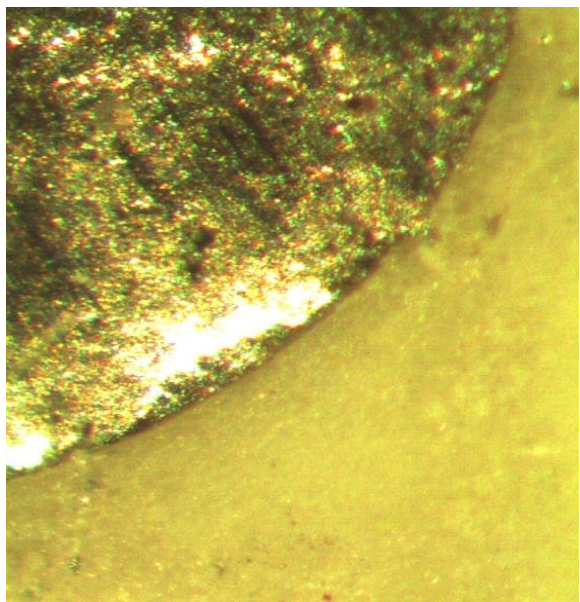

Abb. 28: Gruppe $1 \mathrm{AgF}$ vor Bestrahlung

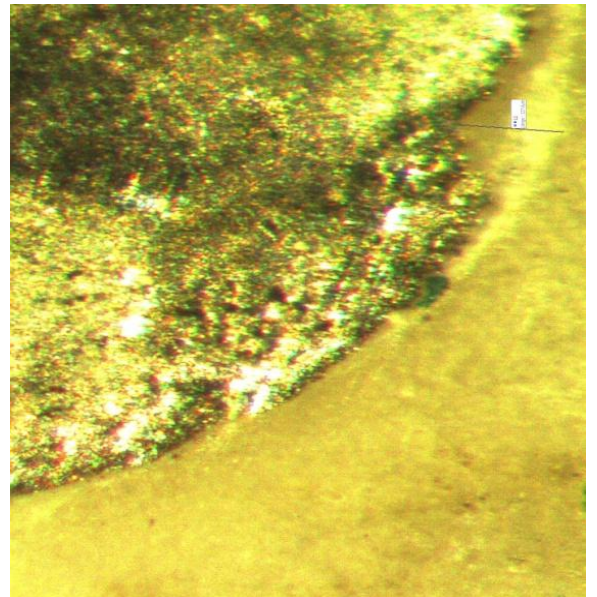

Abb.28a: Gruppe 1 AgF nach BestrahlungSpalt von $127,4 \mu \mathrm{m}$

Auf allen weiteren Bildern waren keine Veränderungen zwischen dem Zustand vor und nach der Bestrahlung zu erkennen:

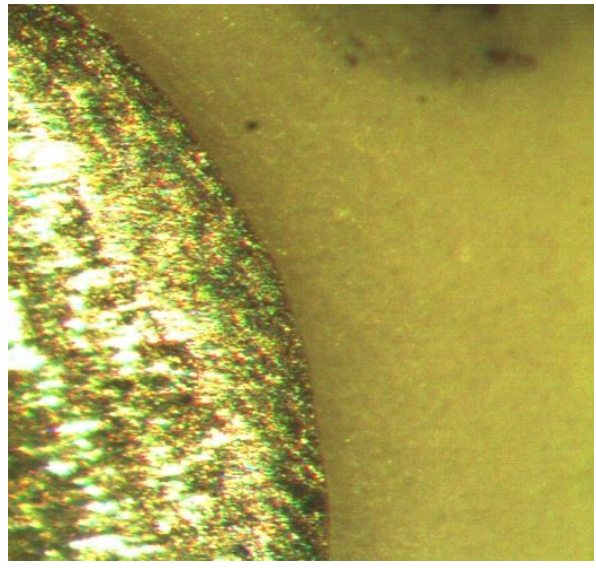

Abb.28b1: Gruppe 1 Amalgamfüllung vor B.

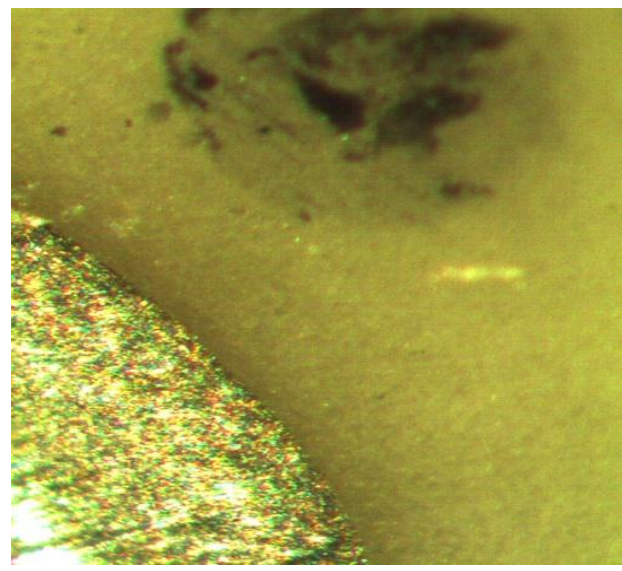

Abb.28b2: Gruppe 1 AgF nach Bestrahlung 


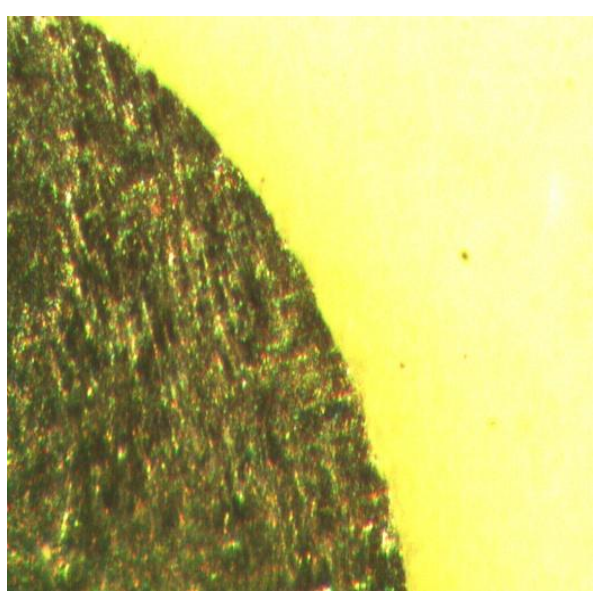

Abb.28c1. Gruppe 1 AgF vor Bestrahlung

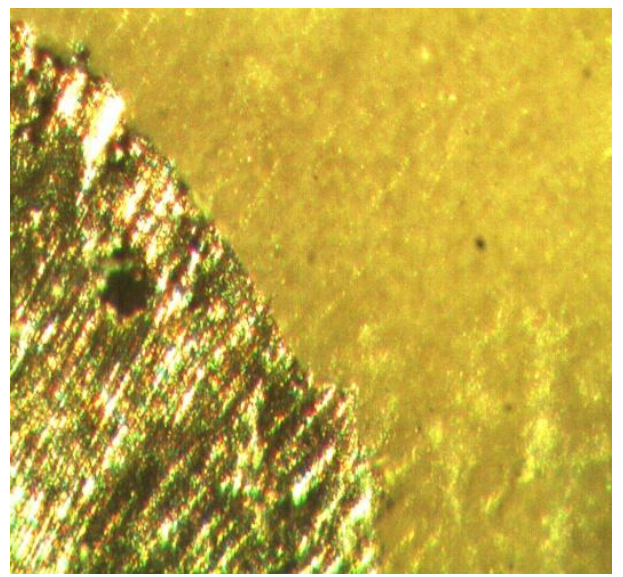

Abb. 28d1: Gruppe 1 AgF vor Bestrahlung

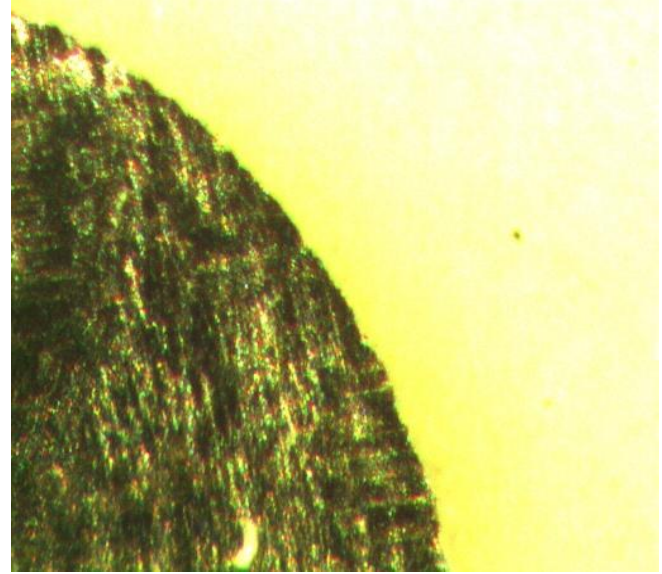

Abb.28c2: Gruppe 1 AgF nach Bestrahlung

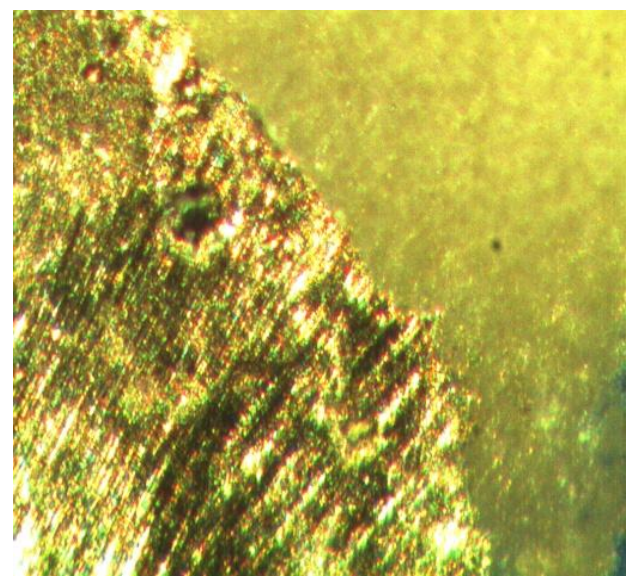

Abb.8d2: Gruppe 1 AgF nach Bestrahlung 
Abbildung 29: Gruppe 2 Amalgamfüllungen mit 90 Gray bestrahlt vor und nach Bestrahlung

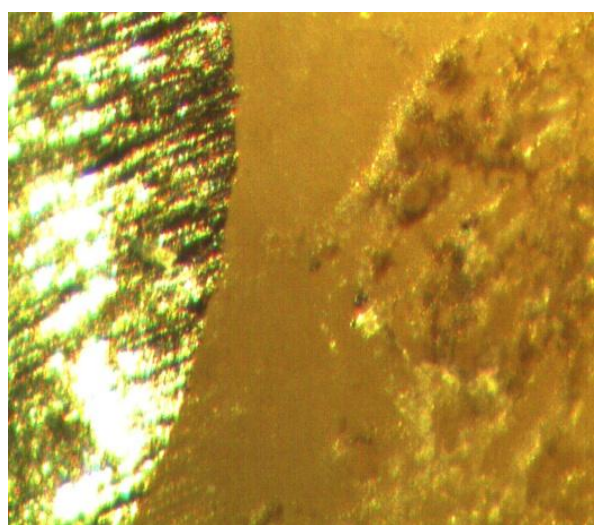

Abb.29: Gruppe 2 AgF vor Bestrahlung

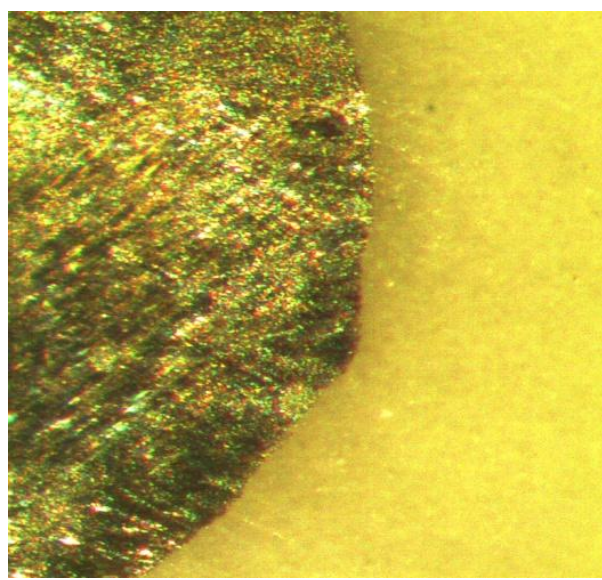

Abb.29b1:Gruppe 2 AgF vor Bestrahlung

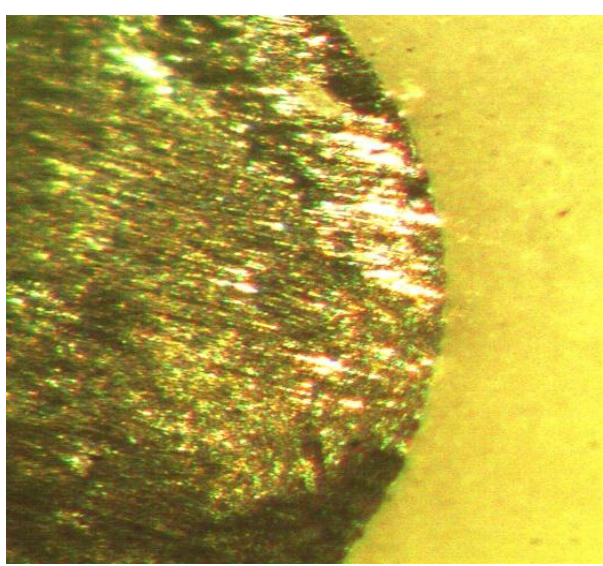

Abb.29c1: Gruppe 2 AgF vor Bestrahlung

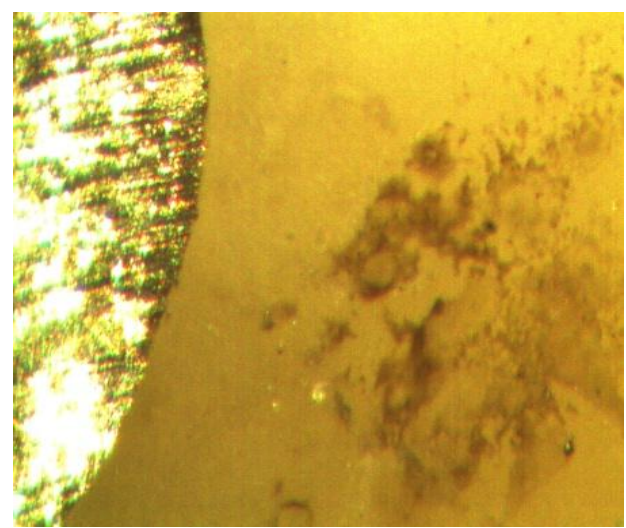

Abb.29a: Gruppe 2 AgF nach Bestrahlung

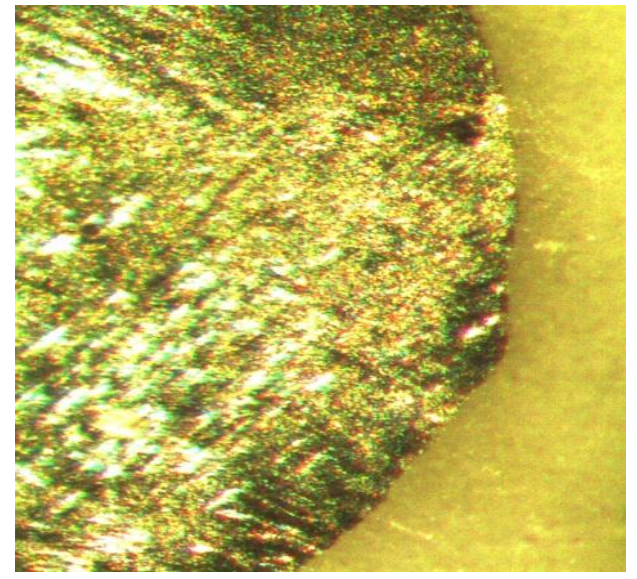

Abb29b2: Gruppe 2 AgF nach Bestrahlung

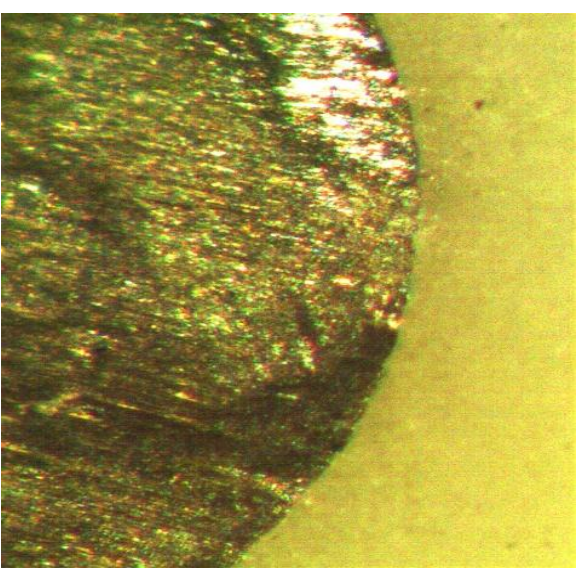

Abb.29c2: Gruppe 2 AgF nach Bestrahlung 
Abbildung 30: Gruppe 3 Kompositfüllungen (KoF) mit 60 Gray bestrahlt vor und nach Bestrahlung

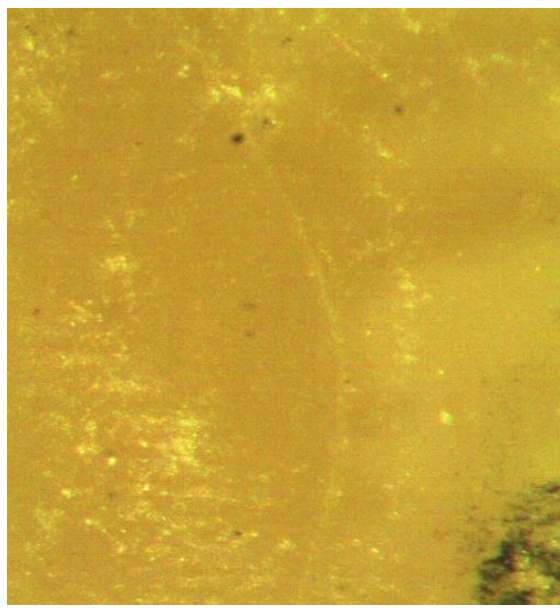

Abb.30: Kompositfüllung Gruppe 3 vor B.

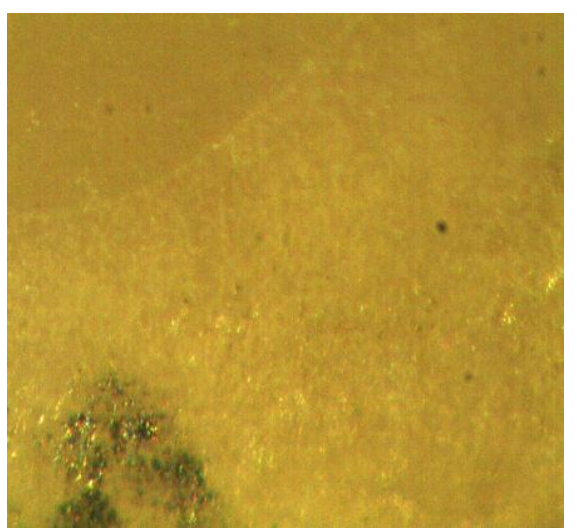

Abb.30b1: Gruppe 3 KoF vor Bestrahlung

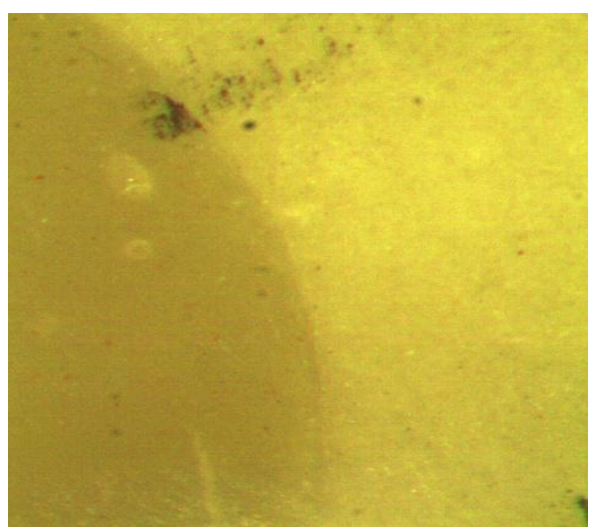

Abb.30c1: KoF Gruppe 3 vor B.

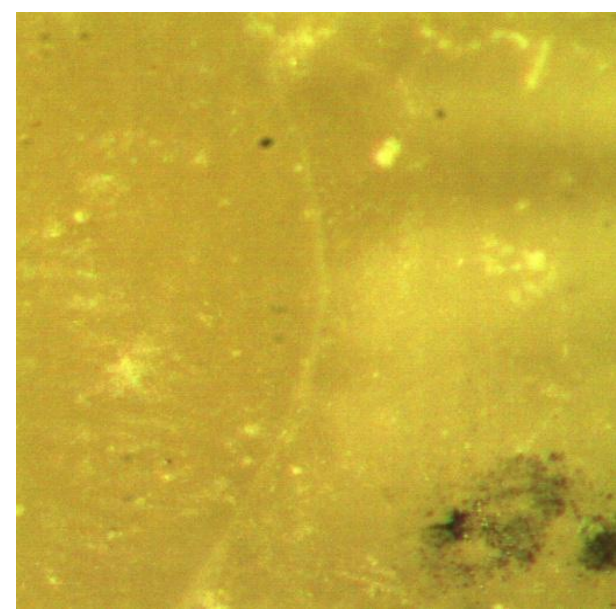

Abb.30a: KoF Gruppe 3 nach Bestrahlung

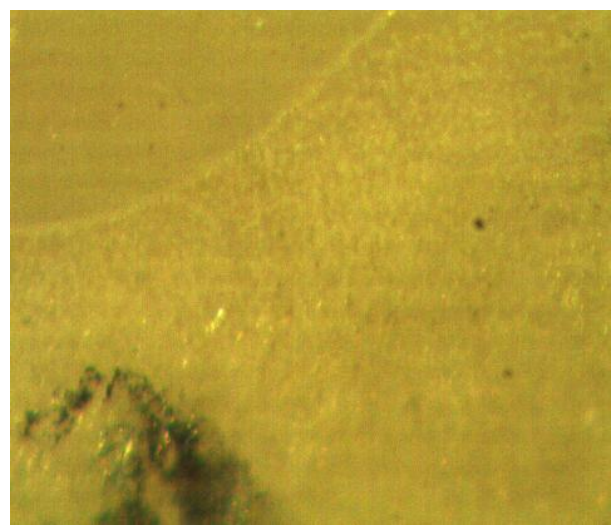

Abb.30b2: Gruppe 3 KoF nach Bestrahlung

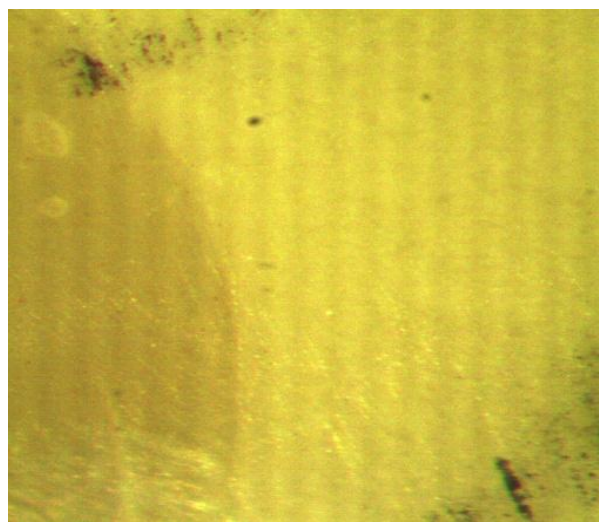

Abb.30c2: KoF Gruppe 3 nach B. 
Abbildung 31: Gruppe 4 Kompositfüllungen mit 90 Gray bestrahlt vor und nach Bestrahlung

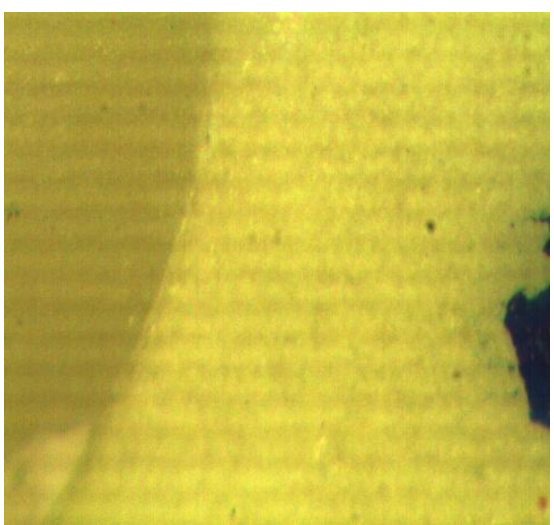

Abb.31:Gruppe 4 KoF vor Bestrahlung

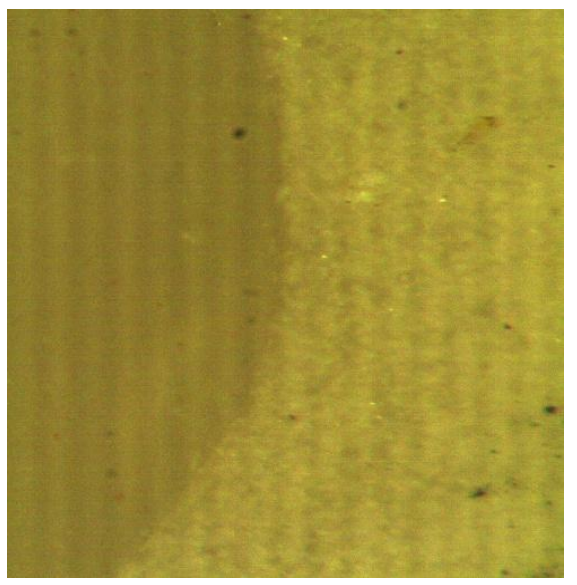

Abb.31b1:Gruppe 4 KoF vor Bestrahlung

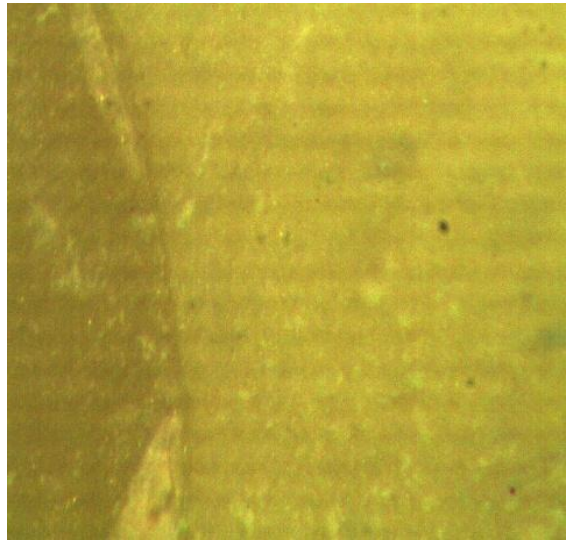

Abb.31c1: Gruppe4 KoF vor Bestrahlung

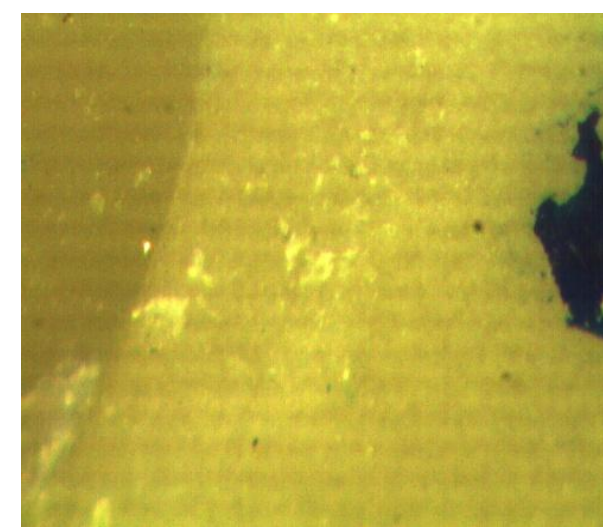

Abb.31a: Gruppe 4 KoF nach Bestrahlung

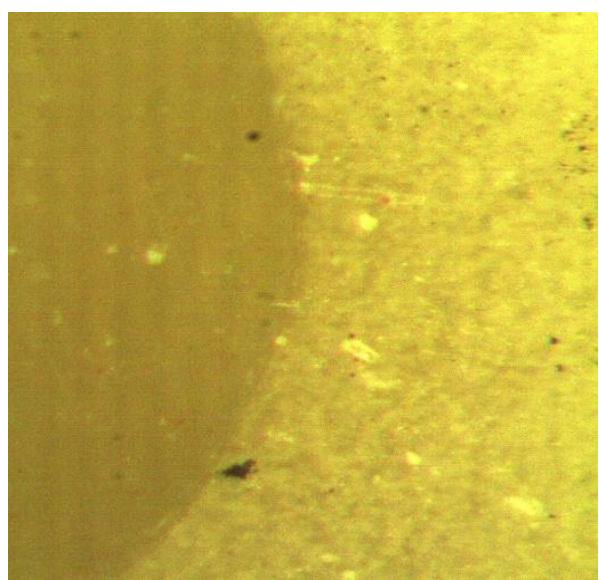

Abb.31b2: Gruppe 4 KoF nach Bestrahlung

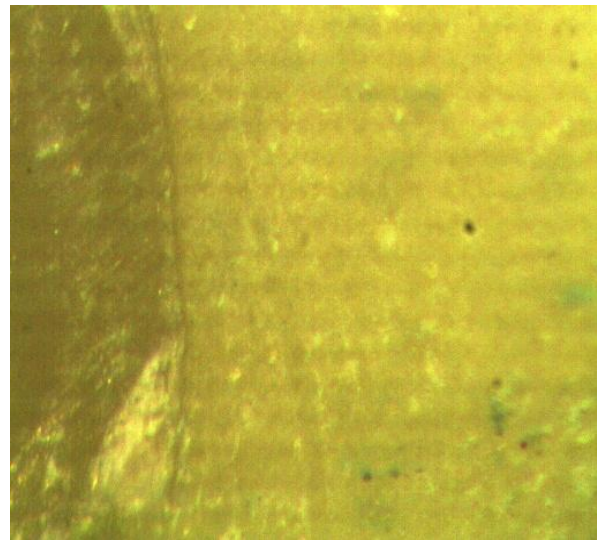

Abb.31c2: Gruppe 4 KoF nach Bestrahlung 


\subsubsection{Ergebnisse der klinischen Untersuchung}

Für eine subjektive klinische Überprüfung wurde mit einer zahnärztlichen Sonde die Grenzfläche Füllung-Zahn abgetastet und vor und nach der Bestrahlung auf einen Spalt untersucht. Auch sollte überprüft werden, ob eine Veränderung zwischen der klinisch verwendeten $60 \mathrm{~Gy}$-Dosis und einer experimentellen Überdosis von $90 \mathrm{~Gy}$ auszumachen ist.

Es konnten bei den Amalgamfüllungen nur bei drei Füllungen der Gruppe 1 und bei einer Füllung der Gruppe 2 nach der Bestrahlung eine Veränderung durch Haken der Sonde festgestellt werden.

Bei den Kompositfüllungen waren es in Gruppe 3 und 4 jeweils vier Füllungen, bei denen ein Unterschied zu vor der Bestrahlung an der zu untersuchten Position zwischen sechs und sieben Uhr zu verzeichnen war (siehe Tabelle 3). Trotz einer Veränderung des Sondierungsbefundes an einigen Füllungsrändern, konnten alle Füllungen in unserer Arbeit nach den Ryge-Kriterien mit einem "alpha" bewertet werden, da die Sonde zwar hakte, visuell jedoch mit Lupenbrille und 2-facher Vergrößerung keine Stufe oder kein Spalt zu erkennen war.

Tabelle 4: Anzahl der bei der klinischen Untersuchung nach der Bestrahlung aufgetretenen Veränderungen

\begin{tabular}{|l|l|l|l|l|}
\hline Material & Gruppe & Dosis & Veränderung: Ja & Veränderung: Nein \\
\hline Amalgam & & 0 Gray & 0 & 10 \\
\cline { 2 - 5 } & 1 & 60 Gray & 3 & 7 \\
\cline { 2 - 5 } & 2 & 90 Gray & 1 & 9 \\
\hline Komposit & & 0 Gray & 0 & 10 \\
\cline { 2 - 5 } & 3 & 60 Gray & 4 & 6 \\
\cline { 2 - 5 } & 4 & 90 Gray & 4 & 6 \\
\hline
\end{tabular}

Statistisch wurde dies mit dem Fisher Exact-Test mit der freien Statistiksoftware R überprüft. Der exakte Fisher-Test betrachtet eine Kontingenztafel, die zeigt, ob aus unterschiedlichen Behandlungen auch unterschiedliche Ergebnisse resultieren. Er stellt keine Voraussetzungen an den Stichprobenumfang und liefert, auch bei wenigen Beobachtungen wie in dieser Studie, zuverlässige Resultate. Seine Nullhypothese sagt aus, dass der 
Einfluss auf das Endergebnis „Verschlechterung ja/nein“ unabhängig von der Behandlung ist. Als Signifikanzniveau wurde $5 \%$ gewählt. Bei $p<0,05$ kann die Nullhypothese also verworfen werden.

Die statistisch angenommene Nullhypothese besagt, dass es keine Auswirkung auf die Qualität des Randspaltes zwischen Füllung und Zahn bezüglich einer Verschlechterung $\mathrm{ja} /$ nein hat, ob eine Probe unbestrahlt ist oder mit einer $60 \mathrm{~Gy}$-Dosis bzw. einer $90 \mathrm{~Gy}-$ Dosis bestrahlt wird.

Eine weitere Nullhypothese besagt, dass es keine Auswirkung auf die Qualität des Randspaltes zwischen Füllung und Zahn bezüglich einer Verschlechterung ja/nein hat, ob eine Probe mit einer $60 \mathrm{~Gy}$-Dosis oder einer $90 \mathrm{~Gy}$-Dosis bestrahlt wird.

Bei allen Vergleichen ist ein p-Wert größer als 0,05 errechnet worden. Bei einem p- Wert kleiner als 0,05 hätte man statistisch einen signifikanten Unterschied zwischen den unbehandelten Proben und den mit der jeweiligen Dosis bestrahlten Proben bezüglich einer Verschlechterung ja/nein gezeigt. Auch wäre im zweiten Fall bei einem p-Wert unter 0,05 ein signifikanter Unterschied zwischen der Dosis von 60 und 90 Gy bezüglich einer Verschlechterung ja/nein deutlich gemacht worden.

Trotz einer Veränderung des Sondierungsbefundes an einigen Füllungsrändern (Tab. 3) konnte eine statistische Signifikanz (Fisher's Exact-Test) nicht gezeigt werden. Die Nullhypothesen werden somit aufrechterhalten. 


\section{Diskussion}

Es liegen bereits zahlreiche Studien vor, die sich mit der rückwärtigen Streustrahlung von Metallen und den Auswirkungen der daraus resultierenden lokal, d.h. in der Mundhöhle, an Implantaten, Rekonstruktionsplatten und anderen zahnärztlichen Materialien auftretenden Dosiserhöhung beschäftigt haben (Chin et al 2009; Farahani et al. 1993; Gagnon und Cundiff 1980; Gibbs et al. 1976; Reitemeier et al. 2002; Thilmann et al. 1995; Wang et al. 1996).

Die Forschung konzentrierte sich dabei jedoch hauptsächlich auf die Wirkung der Strahlung auf die oralen Weichgewebe oder auf die Verursachung von Sekundärkaries mit und ohne Fluoridnutzung.

Offen ist immer noch die Frage, ob sich die Strahlen bzw. die höheren Strahlendosen auch an der Grenze zwischen Füllungswerkstoff und Zahnhartsubstanz auswirken und eventuell deutliche Unterschiede zwischen den metallischen und nicht-metallischen Restaurationsmaterialien zu verzeichnen sind. Es gibt verschiedene in der Radiotherapie genutzte Strahlenarten. Bekannt ist, dass es durch deren Wechselwirkungen mit der Materie zu einer Streuung und Absorption und damit Schwächung der Strahlung kommt. Abhängig von der Höhe der Röntgenspannungen überwiegen der Photoeffekt, der Compton- und der Paarbildungseffekt. Bezüglich der Wechselwirkungen zwischen Strahlung und biologischer Materie sind resultierende biochemische Veränderungen in den Zellen mit Auswirkungen auf deren Funktionen und letztendlich den gesamten Organismus bekannt (Kielbassa 2004). Die damit einhergehenden Nebenwirkungen beinhalten u.a. Mukositis, Hyposalivation, Geschmacksverlust, Osteoradionekrose, Kieferklemme und Strahlenkaries (Dreizen et al. 1977; Rahman et al. 2013; Vissink et al. 2003b). Sowohl direkte wie auch indirekte Strahlenwirkungen auf die Mundhöhle und ihre Strukturen können akut oder chronisch verlaufenden Nebenwirkungen induzieren. 


\subsection{Material}

\subsubsection{Rinderzähne}

Wir haben uns in dieser Studie für Rinderzähne als Probenmaterial entschieden, aus den im Wesentlichen schon von Oesterle et al. (1998) beschriebenen Gründen. Danach liegt der experimentelle Vorteil von Rinderzähnen darin, dass sie einfach und günstig in einer ausreichenden Stückzahl erhältlich sind, dann aber auch im Vergleich mit menschlichen Zähnen ähnlich große ebenmäßige und kariesfreie Oberflächen aufweisen. Die Ähnlichkeit mit menschlichen Zähnen ist besonders bei älteren Rinderzähnen auffällig

(Fonseca et al. 2008). Deshalb achteten wir auch darauf, dass die von uns im Experiment verwendeten Rinderzähne von etwa gleichaltrigen älteren Tieren stammen und auch aus dem gleichen Schlachtbetrieb kamen.

Hinsichtlich der experimentellen Brauchbarkeit von Rinderzähnen wird in der Literatur zudem darauf verwiesen, dass der Haftverbund der Restaurationsmaterialien zum Schmelz und oberflächlichen Dentin keine signifikanten Unterschiede zwischen humanen und bovinen Zähnen erkennen lässt (Nakamichi et al. 1983).

Nach den u.a. von Nakamichi et al. (1983) gewonnenen Ergebnissen konnten wir in unserer Studie somit auch davon ausgehen, dass der Klebeverbund der zahnmedizinischen Materialien zu den bovinen Zahnhartsubstanzen dem Klebeverbund an den menschlichen Zahnhartsubstanzen in etwa entspricht. Um die Entsprechung zu den humanen Zähnen möglichst groß zu halten, haben wir zudem darauf geachtet, dass unsere in die Rinderzähne präparierte Kavitäten ebenfalls nur von Schmelz und oberflächlichem Dentin begrenzt waren.

Auch Almeida et al. (2009) fanden, was das Microleakage der Adhäsivsysteme anbelangt, keinen signifikanten Unterschied zwischen humanem und bovinem Schmelz und Dentin. Auf eine signifikante Vergleichbarkeit von humanen und bovinen Zähnen weist schließlich die Untersuchung von Jansma et al. (1988a) hin. Sie stellten in ihrer In-vitro-Studie fest, dass die Bestrahlung die Säurelöslichkeit des Schmelzes bei Rinderzähnen in vitro herabsetzt. Zu ähnlichen Schlussfolgerungen kamen Pioch et al. (1991) und Grötz et al. (1998) bei menschlichen Zähnen nach Radiotherapie. 
Wir gehen aufgrund der aktuellen Studienlage von der Ähnlichkeit zwischen Rinder- und Menschenzähnen aus. Es ließen sich des Weiteren keine Unterschiede bezüglich des Klebeverbundes oder der Randadaptionen erkennen.

Die Wahl unseres Probenmaterials durfte sich daher mit der Erwartung verbinden, dass das Ergebnis unserer Studie, also das, was sich unter dem Auflichtmikroskop an den Randspalten zeigen sollte, die Übertragung auf die Bestrahlung menschlicher Zähne erlaubt.

\subsubsection{Künstlicher Speichel}

Um die Füllungen und die Rinderzähne in einer möglichst naturgetreuen Lösung zu lagern, nutzten wir die von Klimek et al. für künstlichen Speichel zusammengestellten Substanzen (Klimek et al. 1982).

Es gibt diverse Studien, die beschreiben, dass künstlicher Speichel frühe Schmelzerosionen wieder remineralisieren und die Schmelzhärte stärken kann (Amaechi und Higham 2001; Devlin et al. 2006). In einigen Studien werden allerdings auch andere Lagermedien verwendet. So wurden - neben der Nutzung von Speichel (Kappert et al. 1991) in einigen Studien - die Proben auch nur in Wasser und bei $37^{\circ} \mathrm{C}$ (Ikeda et al. 2002), in säurehaltigen und bakteriellen Medien (Grossman und Matejka 1997) oder in 0,1 \% Thymollösung (Rammelsberg et al. 2000) gelagert.

\subsection{Methoden}

\subsubsection{Vorversuche}

In den Vorversuchen wurde überprüft, ob die Bestrahlung Reaktionen an den einzelnen untersuchten zahnärztlichen Materialien hervorruft.

Für die Hauptversuche wurde ein Verfahren entwickelt, in dem die Kavitäten hauptsächlich im Schmelz und nicht wie in den Vorversuchen vollständig im Dentin positioniert wurden. Dies war wichtig, da die Klebehaftung bei Rinderzähnen hauptsächlich am Schmelz und oberflächlichen Dentin der Haftung an Menschenzähnen ähnelt (Nakamichi et al. 1983). Zudem ist ein standardisiertes Präparations- und Applikationsverfahren der 
Füllungen entwickelt worden, damit diese ebenmäßigere Oberflächen aufwiesen als es in den Vorversuchen der Fall war.

Vom Raster-Elektronen-Mikroskop nahmen wir - aus den bereits in Abschnitt 4.2.1.3 genannten Gründen - Abstand. Wir verzichteten auf die Replikatechnik, da ein weniger aufwendigeres und kostengünstigeres Verfahren in den Vorversuchen angewendet werden sollte. So brauchte sowohl der kühle Sputter wie auch das REM mehrere Stunden, um das benötigte Vakuum zu erlangen. Es wäre jedoch empfehlenswert gewesen, die zu untersuchende Probe mit einem bewährten Silikon abzuformen und die Modelherstellung aus z.B. einem Epoxidharz anzuwenden, um der Zerstörung der Proben durch das REM vorzubeugen und die höhere Vergrößerung des REM nutzen zu können.

Die für das REM notwendigen Verfahren wie Säuberung mit 70-\%igem Alkohol, Trocknung und Besputterung mit Metallen fügten den Zähnen schon vor der Bestrahlung große Schäden zu - unter der hohen Auflösung an zahlreichen Rissen und Sprüngen erkennbar. Um diese Nachteile zu vermeiden, entschieden wir uns für das Auflichtmikroskop.

\subsubsection{Hauptversuche}

Alle unsere Untersuchungen wurden in vitro durchgeführt. Es bleibt somit festzuhalten, dass die Ergebnisse, wie in anderen In-vitro-Studien auch, nur annäherungsweise und nie vollständig auf die entsprechende Situation in vivo zu übertragen sind (Pashley 1991). Schon kleine Unterschiede in der Verarbeitung der Materialien sowie in den biologischen Eigenschaften (Sklerosierung des Dentins) können zu anderen Ergebnissen führen. Außerdem ist zu bedenken, dass besonders bei der adhäsiven Technik eine völlig randspaltfreie Adaption eines restaurativen Materials auf die Zahnhartsubstanz sowohl unter Invivo- wie unter In-vitro-Bedingungen schwierig zu prognostizieren ist (Hannig und Friedrichs 2001).

\subsubsection{Herstellung der Proben}

Die Rinderzähne sind gründlich mit Skalpellen, scharfen Löffeln und Scalern von Geweberesten und oberflächlichen Verunreinigungen befreit worden. Dieses Vorgehen wäre vermutlich mit einem Pulverstrahlgerät stark vereinfacht worden, jedoch hätten hierbei 
Oberflächenveränderungen die Folge sein können. Somit entschieden wir uns zur Säuberung der Rinderzähne für die Handinstrumente.

Auch war es uns bei dieser Studie sehr wichtig, standardisierte Verhältnisse zu schaffen. Denn die Standardisierung der Versuchsbedingungen bleibt ein entscheidendes Kriterium, um die gewonnenen Ergebnisse gut miteinander vergleichen zu können. Damit eine definierte Tiefe und Breite der Kavitäten gewährleistet war und mögliche von Hand verursachte Fehler vermieden werden konnten, wurden die verwendeten Diamantbohrer in eine einheitlich funktionierende Bohrmaschine eingespannt. Die Ausdehnung der Kavitäten konnte so bei allen Proben genau gleich gestaltet werden.

Um diese identische Ausdehnung der Kavitäten in Breite und Tiefe garantieren zu können, wurde zudem für eine definierte Höhe der eingebetteten Rinderzähne gesorgt. Hierzu ließen wir eine Gießform in der wissenschaftlichen Werkstatt der Universitätsmedizin Göttingen anfertigen und betteten die Zähne in einer streng abgemessenen Menge des Kaltpolymerisats Paladur ${ }^{\circledR}$ ein. In dieser Gießform ragten die großen, glatten, kariesfreien bukkalen und lingualen Schmelzoberflächen schließlich ebenmäßig, in einer einheitlichen Höhe, aus dem Kunststoff heraus.

Bei der Verwendung von extrahierten Zähnen ist es schwierig, unterschiedliche Lagerungszeiten zu vermeiden. Dies schien uns jedoch vertretbar, angesichts von Untersuchungen, die belegen, dass der Zeitraum zwischen Extraktion, Anlegung der Kavitäten und Applikation der Füllungsmaterialien keinen entscheidenden Einfluss auf die In-vitroUntersuchungsergebnisse hat (Blunck et al. 1990). Somit konnte im Rahmen unserer Studie davon ausgegangen werden, dass der Zeitpunkt der Füllungsversorgung keinen positiv verfälschenden Einfluss auf die Randspalten haben würde.

\subsubsection{Auswahl und Verarbeitung der Füllungsmaterialien}

Amalgam und Komposit wurden in dieser Studie als Füllungsmaterialien ausgewählt. Beide Materialien gehören zu den meist verwendeten Werkstoffen in der konservierenden Zahnheilkunde. So gibt es zahlreiche Studien, wie z.B. die von Gibbs et al., Thilmann et al. und Reitemeier et al. (Gibbs et al. 1976; Reitemeier et al. 2002; Thilmann et al. 1995), die die Auswirkung von Strahlung auf das metallische Amalgam untersuchten. Ebenfalls diente in weiteren Studien das zahnfarbene Komposit und seine Klebehaftung an den Hartsubstanzen als Untersuchungsmaterial bei Strahlentherapie (Biscaro et al. 2009; Naves et 
al. 2012; Reitemeier et al. 2002). Dieser Stand der Wissenschaft legte uns die Auswahl der beiden Materialien nahe.

Die Unterschiede der Material- und Verarbeitungseigenschaften dieser beiden Werkstoffe sind groß. Das erschwerte in unserer Studie u.a. die Beurteilung der mikroskopischen Aufnahmen der Randspalten unter dem Auflichtmikroskop vor und nach Bestrahlung. Eine ähnliche Problematik wurde von Duncalf und Wilson (2001) beschrieben, die in ihrer Studie den Randschluss von Amalgam- und Kompositfüllungen überprüften. Sie kamen zu dem Ergebnis, dass diese beiden Materialien eine stark unterschiedliche Ausgangsqualität besitzen, sodass der klinische Vergleich schwierig sei. Duncalf und Wilson (2001) nutzten ebenfalls ein Lichtmikroskop, jedoch mit einer nur 30-fachen Vergrößerung. Um überprüfen zu können, ob unter dem Lichtmikroskop nicht doch eine Veränderung sichtbar wird, wählten wir eine doppelt so hohe Vergrößerung.

\section{Amalgam}

Amalgam gehört in der Zahnmedizin seit über einem Jahrhundert zu den metallischen Füllungsmaterialien der Wahl (Rasines Alcaraz et al. 2014) und ist trotz stetigem Rückgang weltweit immer noch das am meisten verwendete Restaurationsmaterial für die Molaren (Mitchell et al. 2007).

Amalgam ist auch in einigen Studien zu den Rückstreueffekten bei Bestrahlung im Kopfund Halsbereich bereits als metallischer Probenwerkstoff verwendet worden (Gibbs et al. 1976; Thilmann et al. 1995). Diese Studien gaben den lokalen Dosisanstieg an Amalgamfüllungen mit $109 \%$ - $118 \%$ und $161 \%$ an. Es legt sich demnach die Vermutung nahe, dass durch derart hohe Dosen, verursacht durch die Rückstreuung, Veränderungen zwischen der Hartsubstanz und den Füllungsmaterialen auftreten. Gestützt wurde diese Vermutung dadurch, dass es zu einer Veränderung der Schmelzmatrix (Pioch et al. 1991) (Gonçalves et al. 2014) und zu einem veränderten Demineralisationsverhalten (Grötz et al. 1998) infolge der energiereichen Strahlen kommt. Auf dieser Basis führten wir unsere Untersuchungen durch.

Die Applikation der Amalgamfüllungen erfolgte in der nach Herstellerangaben vorgeschriebenen Vorgehensweise. Die Misch- und Verarbeitungszeit von 10 Sekunden bzw. 3 Minuten wurde eingehalten. Die abschließende Brünierung und Politur der Amalgamfül- 
lungen wurde ebenfalls sehr sorgfältig und nach Richtlinien der Hersteller durchgeführt, da die gründliche Beendigung einer Amalgamfüllung - im Vergleich zu den Oberflächen unpolierter Amalgamfüllungen - marginale Diskrepanzen, die möglicherweise auftreten, stark vermindert (Wöstmann und Lütke-Notarp 1991). Die achtsame Verarbeitung und sorgfältige Applizierung der Füllungsmaterialien in die Kavitäten ist von großer Bedeutung, da durch falsche Arbeitsvorgänge oder Materialüberschüsse ungenügende Randadaptionen der Materialien an die Hartsubstanzen die Folge sein können, was letztendlich zu einer geringeren Lebensdauer der Füllungen führt (Palotie und Vehkalahti 2002). Auch hätte sich sonst die Frage stellen können, ob die auftretenden Randspalten auf die Bestrahlung oder die unsaubere Verarbeitung der Materialien zurückzuführen sind. Eine mögliche Kritik an der Verarbeitung der Füllungsmaterialien sollte von vornherein ausgeschlossen werden. An diesem Punkt muss ebenfalls angemerkt werden, dass der Standard der applizierten Amalgamfüllungen in vivo natürlich häufig geringer ausfällt und wir somit nicht wissen, ob nicht mindere Qualitäten von Amalgamfüllungen ganz anders auf die Strahlen reagieren.

Die Lebensdauer der Füllungsmaterialien hängt von verschiedenen Faktoren ab, vor allem von der Materialeigenschaft, dem Patient und dem Zahnarzt (Hickel und Manhart 2001). Wir konnten diese Einflussfaktoren in dieser Studie jedoch vernachlässigen, da durch unsere standardisierten Verhältnisse und die Nutzung der gleichen und bewährten Materialien stets ähnliche Begebenheiten in vitro geschaffen werden konnten.

Es ist jedoch nicht gänzlich auszuschließen, dass es durch die erleichterte Applikation der Füllungsmaterialien bei diesem In-vitro-Verfahren, z.B. durch die verbesserte Sicht, zu einer besseren Adaption der Materialien an den Hartsubstanzen gekommen ist als es bei In-vivo-Füllungslegungen, unter erschwerten Begebenheiten in der Mundhöhle, der Fall gewesen wäre. Alptekin et al. (2010) widerlegten allerdings diese Vermutung, in dem sie die Randadaption von in vivo und in vitro applizierten Komposit- und Amalgamfüllungen untersuchten und zu dem Schluss kamen, dass es keine signifikanten Unterschiede zwischen in vivo und in vitro applizierten Füllungen gibt.

Alptekin et al. (2010) stellten nur fest, dass Randspalten bei Komposit mit höherer Wahrscheinlichkeit auftreten als bei Amalgam. Diese Aussage konnten wir mit unseren mikroskopischen Ergebnissen allerdings nicht bestätigen. Unter dem hier genutzten Auflichtmikroskop waren bei 66-facher Vergrößerung vor und nach Bestrahlung Randspalten weder 
bei Amalgam noch bei Komposit zu erkennen. Zu einem ähnlichen Ergebnis, nämlich, dass Komposit nicht zu höheren Fehlerraten als Amalgam führt, kam auch Hurst (2014).

Komposit

Als zahnfarbenes Füllungsmaterial wurde in dieser Studie Venus ${ }^{\circledR}$ als FeinstpartikelHybridkomposit verwendet. Mit dem steigenden Bewusstsein für die eigene Mundgesundheit und für die Zahn-Ästhetik wächst in der Bevölkerung der Wunsch nach zahnfarbenen Alternativen. Es gibt heute eine große Vielzahl von unterschiedlichsten Kompositmaterialien, die sich in mechanischen und anwendungsspezifischen Eigenschaften sowie in ästhetischen Möglichkeiten unterscheiden, bei richtiger Anwendung aber alle einen hohen Qualitätsstandard und eine gute Haltbarkeit besitzen (Ferracane 2011). Es war uns wichtig, dieses nicht-metallische Material ebenfalls zu untersuchen, auch wenn aufgrund fehlender Studien zu vermuten stand, dass es bei Kunststoff wegen dessen geringeren Dichte und niedrigeren Ordnungszahl zu keiner hohen rückwärtigen Streustrahlung und folglich keiner Dosiserhöhung während der Radiatio kommt. In einer Untersuchung des Universitätsklinikums Münster wurde auch beschrieben, dass es durch die Weiterentwicklung verschiedener Keramikarten und Kunststoffe eine Vielzahl von alternativen Versorgungsmöglichkeiten gibt (Suwelack et al. 2014). Sie untersuchten die Rückstreuung des prothetischen Biomaterials Keramik im Vergleich zu den in vielen Studien genutzten metallischen Materialien. Sie konnten darstellen, dass die Dosiserhöhung von 5,67 \% bei Keramik in der Tat sehr viel geringer ausfällt als die bei Goldlegierungen von $25 \%$. Durch ihre Studie sahen wir uns bestärkt darin, auch die heute beliebten alternativen zahnfarbenen Werkstoffe auf ihre Qualität und Beständigkeit nach der Bestrahlung zu untersuchen. Außerdem beschrieben Naves et al. (2012)den für uns interessanten Tatbestand, dass Röntgenstrahlen einen deutlich nachteiligen Effekt auf den Halt von Füllungsmaterialien zu Schmelz und Dentin hatten, sofern die adhäsiven Restaurationen nach der Radiotherapie angefertigt wurden.

Das in unserer Studie verwendete Optibond $\mathrm{FL}^{\circledR}$ als Adhäsivsystem und Haftvermittler zwischen der hydrophilen Zahnhartsubstanz und dem hydrophoben Komposit ist ein Zwei-Komponenten-Adhäsivsystem. Der oberflächenkonditionierende Primer basiert auf Wasser-Alkohol-Komponenten. Optibond $\mathrm{FL}^{\circledR}$ wird mittlerweile als "Goldstandard" be- 
schrieben und zeigt weit bessere Ergebnisse in Studien (Peumans et al. 2012) als Einflaschensysteme (Frankenberger and Tay 2005).

Frankenberger et al. (2007) stellten auch fest, dass die Randadaption bei klebenden Restaurationen in In-vitro-Studien sehr zuverlässig prognostizierbar ist, wenn eine gute Simulation der In-vivo-Umstände stattfindet. Dennoch fallen die Beurteilungen der Randqualitäten bei direkten Restaurationen in vitro schlechter aus als die in vivo noch als klinisch akzeptabel beurteilten Fälle. Auch wenn in unserer Studie den In-vivo-Begebenheiten noch genauer hätte entsprochen werden können, ist bei unserer mikroskopischen Auswertung der Randspalten die zu strenge Bewertung in vitro nicht eigens zu diskutieren, da fast alle Füllungen lichtmikroskopisch eine gute Adaption aufzeigten.

Auch die Verarbeitung des Kompositmaterials erfolgte nach Herstellerangaben. Obendrein wurde die vielseitig beschriebene Inkrementtechnik angewendet, um den Polymerationsschrumpfungen des Materials vorzubeugen. Zu verweisen ist diesbezüglich auf Studien, die behaupten, dass die Schichttechnik nur bei größeren Kavitäten diese Vorteile erbringen würde (He et al. 2008), sie folglich bei unseren kleinen Kavitäten gar nicht notwendig gewesen wäre.

In diesem Zusammenhang ist zudem darauf hinzuweisen, dass die Füllungen in unserer Studie unter optimalen Bedingungen gelegt worden sind. Dies kann, wie schon angesprochen, unter demgegenüber erschwerten klinischen Bedingungen in vivo und bei Patienten mit den unterschiedlichsten Mundhygienegewohnheiten und kariesaktiven Gebissen nicht immer der Fall sein.

Dahin gehend ist es durchaus möglich, dass klinisch die Situation sich doch etwas anders und gegebenenfalls weniger positiv darstellt.

\section{Erweiterung der Methode}

Crim und Mattingly (1981) haben darauf hingewiesen, dass In-vitro-Studien die oralen Begebenheiten so ähnlich wie möglich simulieren sollten.

Dem folgend sollten in weiteren Studien, die auf unserem neu etablierten Versuchsdesign aufbauen, die Füllungen zusätzlich einer thermischen Wechselbadbelastung von 2000 Zyklen ausgesetzt werden. Diese 2000 Zyklen einer thermischen Wechselbadbelastung zwischen $5^{\circ} \mathrm{C}$ und $55^{\circ} \mathrm{C}$ entsprechen in etwa einer In-vivo-Belastung von zwei Jahren und 
gelten als realistische Belastungsmethode, denen die Füllungen in vivo ausgesetzt sind (Crim et al. 1985; Noack 1988). Bei Nutzung des Thermocyclings könnte es trotz Vorhandenseins einer initial guten marginalen Adaption im Anschluss an das Wechselbad zur Bildung von Randspalten kommen.

In einer Folgestudie sollte auch als zusätzliches Füllungsmaterial noch Glasionomerzement (GIZ) untersucht werden. Die Glasionomerzemente bestehen aus den für die Dentalzemente typischen Bestandteilen Pulver und Flüssigkeit und härten durch eine SäureBase-Reaktion aus (Hellwig et al. 2009). Es gibt sie in verschiedenen Ausführungen (Konventionelle GIZ, Cermetzemente, Hochvisköse und Kunststoffmodifizierte GIZ). Aufgrund ihrer Fluoridabgabe werden die GIZ häufig in kariesaktiven Gebissen und in der Kinderzahnheilkunde eingesetzt (Hellwig et al. 2009).

Das beschriebene Füllungsmaterial erlangte in vielen Studien eine positive Resonanz, insbesondere wenn die Patienten während und nach ihrer Strahlentherapie Nachlässigkeit in der regelmäßigen Fluoridgelnutzung zeigten (De Moor et al. 2011; Haveman et al. 2003; McComb et al. 2002). Da die GIZ eine geringere Abrasivität und strukturelle Integrität aufweisen als andere Materialien (De Moor et al. 2011) wäre es interessant, in einer weiteren Studie auch seine Beständigkeit und Randqualität vor und nach Bestrahlung zu untersuchen.

\subsubsection{Mikroskopie und Messung der Proben}

Die Passgenauigkeit einer zahnärztlichen Versorgung ist ein entscheidendes Kriterium für den Langzeiterfolg einer Restauration. Je geringer die Qualität einer Füllung ist, also je schlechter die Passung, desto höher ist das Risiko für das Auftreten einer Sekundärkaries (Goldberg et al. 1981). Da die Sekundärkaries der größte Hauptverursacher für den Verlust von Restaurationsmaterialien, also die begrenzte Langlebigkeit, ist (Collins et al. 1998; Palotie und Vehkalahti 2002), ist die genaue Passung einer Füllung sehr bedeutend.

Um die Randqualität der Komposit - und Amalgamfüllungen vor und nach der Bestrahlung mit 60 und 90 Gy und dem Auftreten eventueller strahlenbedingter Veränderungen zu untersuchen, beurteilten wir in dieser Studie die Grenzfläche Zahn-Füllung unter dem Auflichtmikroskop bei 66-facher Vergrößerung.

Zur Auswertung und Beurteilung der Randqualität von zahnärztlichen Restaurationen stehen funktionelle und morphologische Untersuchungsmethoden zur Verfügung, wobei die 
Letzteren noch einmal in direkte und indirekte morphologische Methoden unterteilt werden.

Die morphologischen Verfahren haben den Vorteil, dass sie keinen alterierenden Behandlungen unterzogen werden. So eignen sich diese Untersuchungsmethoden sowohl für Invivo- als auch für In-vitro-Studien. Außerdem sind Verlaufsuntersuchungen mit den morphologischen Methoden möglich, weshalb in der vorliegenden Arbeit ausschließlich die morphologischen Untersuchungsmethoden gewählt wurden.

\section{Klinisch mit Sonde und Spiegel}

Die direkte morphologische Methode mit Sonde und Spiegel, die hier auch verwendet wurde, gilt als eine der einfachen Methoden. Es wird jedoch auch bei strikt gleich angewendeten Beurteilungskriterien die mangelnde Objektivierbarkeit und Quantifizierbarkeit als nicht zufrieden stellend bewertet (Dünninger et al. 1991). Ryge et al. (1981) dagegen beschrieben, bei Anwendung gleicher Kriterien, eine sehr hohe Übereinstimmung, selbst wenn unterschiedliche Personen die Bewertung vornehmen.

Bei Kreulen et al. (1993) wurden die Methoden zur Randpassung in direkte, mit Sonde und Spiegel, und indirekte Methoden, die Fotografien der Impressionen der Ränder in Replikamodellen einschließen, unterteilt.

Die indirekte Methode der Replikatechnik, mit einem zusätzlich vorliegenden Messbalken im REM, wurde als aussagekräftiger eingestuft, besonders wenn nur geringe Unterschiede des marginalen Spaltes zu erwarten sind (Kreulen et al. 1993).

Da heute die Füllungsmaterialien so weit entwickelt sind, dass allenfalls noch geringe Randspalten auftreten, war in dieser Studie davon auszugehen, dass idealerweise mit den einfachen klinischen Instrumenten keine signifikanten Unterschiede erkennbar sein sollten.

\section{Lichtmikroskopie}

Die Lichtmikroskopie, die in dieser Studie eingesetzt wurde, gehört zu den morphologischen direkten Verfahren. Sie zählt ebenfalls zu den einfacheren Methoden, da bei ihr u.a. keinerlei Vorbehandlung der Proben und kein großer apparativer Aufwand notwendig 
ist. Es gilt jedoch als Nachteil, dass hier die Tiefenschärfe nicht sehr gut beurteilt werden kann. Die Tiefenschärfe ist ein Maß für die Ausdehnung des scharfen Bereichs im Objekttraum eines abbildenden optischen Systems. Neben der Tiefenschärfe ist die Vergrößerung in den Auflichtmikroskopen weitaus geringer, verglichen mit den für die quantitative Randanalyse zur Verfügung stehenden Raster-Elektronen-Mikroskopen.

Außerdem wird durch die beim Lichtmikroskop zusätzlich benötigte externe Lichtquelle die Lichtintensität nicht gleichmäßig verteilt. Es werden viele Abschnitte zu dunkel oder zu hell dargestellt. Teile des Objektes sind also nur schwer beurteilbar, da sie das Licht reflektieren oder nur schlecht beleuchtet werden. Dies war auch bei einigen unserer Aufnahmen nicht zu vermeiden.

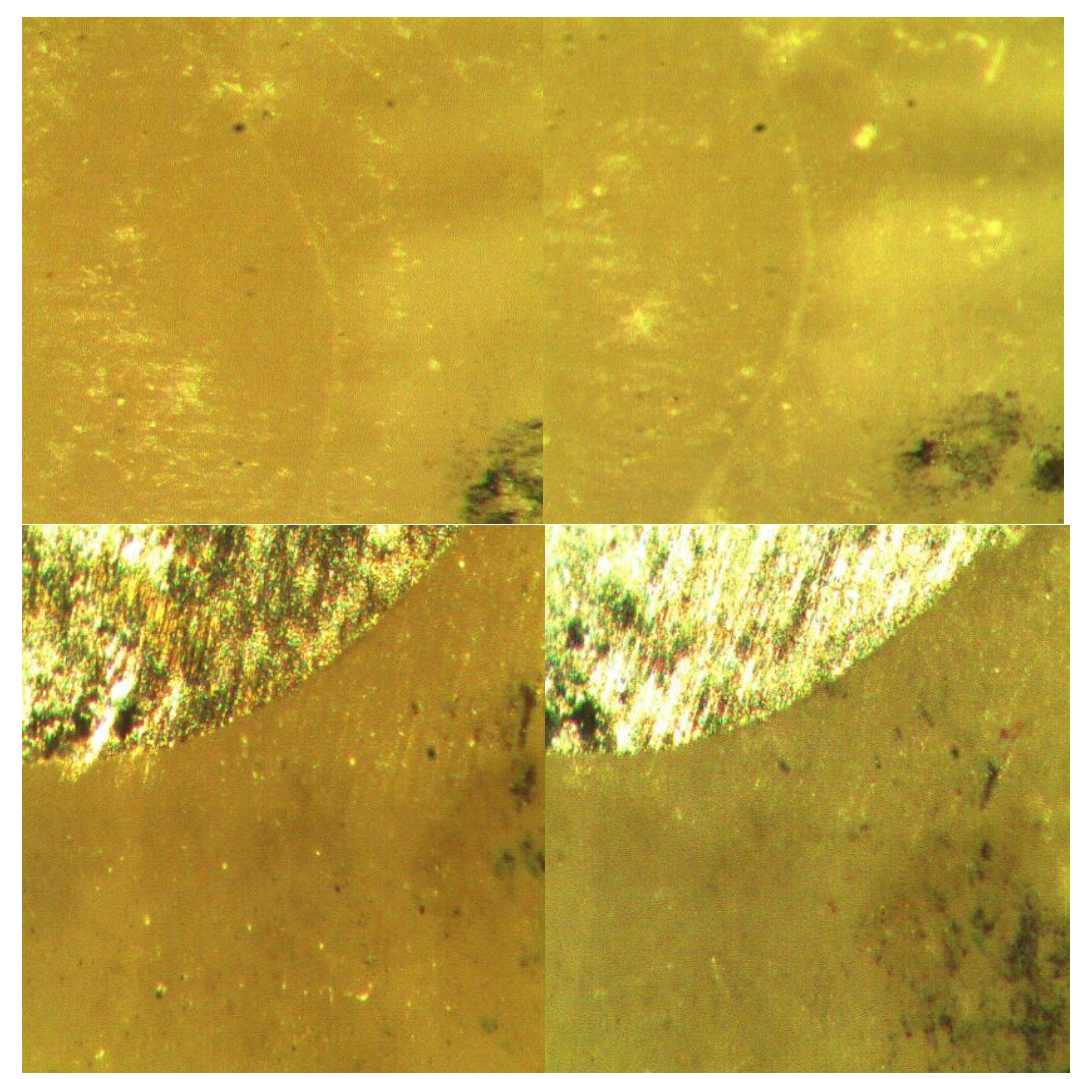

Abb.30: Beispiele für die reflektierten, zu hell oder zu dunkel dargestellten Abschnitte bei den Kompositund Amalgamfüllungen

Auch einige andere Autoren verwendeten die Lichtmikroskopie in Randspaltstudien, so z.B. Duncalf und Wilson (2001), die die Lichtmikroskopie mit einer 30-fachen Vergrößerung nutzten, um die marginale Adaption von Amalgam- und Kompositfüllungsmaterialien in Klasse-II-Restaurationen zu vergleichen. Asmussen und Jorgensen (1972) untersuchten mit dem Lichtmikroskop in ihrer Studie Füllungsadaptionen an Kavitätenwänden. Wöst- 
mann und Lütke-Notarp (1991) untersuchten bei 20-facher Vergrößerung die Randständigkeit "durschschnittlicher" Amalgamfüllungen. So wurde diese Methode auch für die vorliegende Arbeit ausgewählt, um mit möglichst einfachen Mitteln schon eventuell auftretende Veränderungen erkennen zu können. Verglichen mit den zuletzt genannten Studien ist das in dieser Arbeit verwendete Auflichtmikroskop mit 66-facher Vergrößerung, was die Erkennung möglicher Veränderungen an den Randspalten der Füllung betrifft, als aussagekräftiger einzustufen.

\section{Farbstoffpenetrationstest}

Der Farbstoffpenetrationstest gehört zur häufig in Randspaltanalysen genutzten funktionellen Untersuchungsmethode. Hier können mit Hilfe von Farbstoffen, z.B. Anilinfarbstoff, Eosin oder Methylviolett und anschließend angefertigten Schnitten kleinste Spalten durch Penetration der Farbstoffe in den Randspalt zwischen Füllung und Zahnhartsubstanz im nur einfachen Lichtmikroskop nachgewiesen werden (Going 1972; Taylor und Lynch 1992; Wang und Spencer 2005).

Der Farbstoffpenetrationstest benötigt zwar kein Vakuum, Dehydratation oder Kunststoffeinbettung wie sie das REM fordert, erlaubt jedoch auch keine weiterführenden Beurteilungen bzw. Betrachtungen vor und nach Bestrahlung, da der Farbstoffpenetrationstest im Allgemeinen zu einer irreversiblen Veränderung und Zerstörung der Proben führt.

Raster-Elektronen-Mikroskopie

Als morphologische indirekte Methode hat sich für die Untersuchung der Randqualität die quantitative Randanalyse im Raster-Elektronen-Mikroskop in Verbindung mit der Replikatechnik bewährt (Roulet et al. 1989). Durch Herstellung von Epoxidharzreplika entstehen gut duplizierte Probenkörper, die durch die Vakuumbedingungen und Besputterung mit Metallen keinen Schaden nehmen. Die meist bei 200-facher Vergrößerung betrachteten Kavitätenränder werden nach einem Kriterienkatalog definierte Randqualitäten mit Noten zugeordnet (Blunck und Roulet 1989). Neben dieser quantitativen Beurteilung ist außerdem als sehr positiv an dieser Methode zu bewerten, dass sie gut reproduzierbar und 
einfach für Langzeitstudien zu archivieren ist. Dies kann für weitere, auf der unseren aufbauende Studien ebenfalls ein wichtiger Hinweis sein.

Um eine gleichbleibende Qualität der Beurteilung zu erlangen und so Artefakte, z.B. durch die Präparation, richtig zu erkennen ist jedoch eine sachkundige und qualifizierte Auswertung durch einen Experten sehr wichtig (van Meerbeek et al. 2000).

Sehr kritisch beurteilt Heintze (2007) die quantitativen Randanalysen in vitro und fordert, dass die Forschung sich auf weitere Tests konzentrieren sollte, die sich dazu eignen, das klinische Verhalten noch zufriedenstellender vorauszusagen.

Dennoch sollte angesichts des jetzigen Forschungsstandes bei weiteren Untersuchungen zu den Veränderungen von zahnärztlichen Materialien durch die Bestrahlung das REM aufgrund seiner guten klinischen Ergebnisse und Reproduzierbarkeit dem Lichtmikroskop mit seinen Limitationen vorgezogen werden. Bei Nutzung der Replikatechnik und der hohen Vergrößerung im REM wären eventuell doch noch Veränderungen an der Grenzfläche Füllung-Zahn zu erkennen. Zu berücksichtigen wären dabei außerdem die oben bereits vorgeschlagenen alternativen Untersuchungsmöglichkeiten.

\subsubsection{Klinische Untersuchung}

Die Beurteilung mittels Lupenbrille, Spiegel und Sonde gehört ebenfalls zu den morphologischen, aber direkten Untersuchungsmethoden. Sie gilt als sehr subjektiv, nur schwer zu standardisieren und kommt unter anderem bei großen klinischen Studien zum Einsatz (Ryge et al. 1981).

Mit dieser Methode sollte in dieser Arbeit ein einfaches zusätzliches Verfahren angewendet werden, um strahlenbedingte Veränderungen eventuell mit einer zahnärztlichen Sonde ertasten zu können. In Anwendung dieser Methode wurde versucht nach den 1971 von Cvar und Ryge beschriebenen und 2005 wiederveröffentlichten, definierten Kriterien (Cvar und Ryge 2005) vorzugehen. Viele Studien machten von diesen Kriterien Gebrauch (Dünninger et al. 1991; Holan et al. 1996). Trotz einer Veränderung des Sondierungsbefundes an einigen Füllungsrändern, konnten alle Füllungen in unserer Arbeit nach Cvar und Ryge mit der Note "Alpha" bewertet werden, da die Sonde zwar hakte, visuell jedoch mit Lupenbrille und 2-facher Vergrößerung keine Stufe oder kein Spalt zu erkennen waren. Die Adaption des Restaurationsmaterials an der Hartsubstanz schien also zufriedenstellend zu sein und nur leichte Unebenheiten waren mit der Sonde zu ertasten. 
Ryge et al. (1981) beschrieben bei Verwendung gleicher Kriterien eine sehr hohe Übereinstimmung zwischen den Untersuchern. Da in dieser Arbeit weder eine zweite oder dritte Person die Überprüfung vornahm, noch das von Cvar und Ryge geforderte Training der Untersucher erfolgte, ist von einem subjektiven Einfluss auf die Beurteilung der Füllungsränder auszugehen. Dennoch hielten Dünninger et al. (1991) fest, dass die Übereinstimmungen größer werden je positiver die Beurteilungen ausfallen. Da bei den vorliegenden Füllungen alle die Note „Alpha“ bekommen haben, kann trotz allem eine höhere Deckungsgleichheit vermutet werden.

\subsubsection{Bestrahlung}

Die Bestrahlung mittels der experimentellen Röntgenstrahlanlage vollzog sich in der für klinische Zwecke genutzten üblichen Gesamtdosis von 60 Gy und in einer experimentellen Dosis von 90 Gy. Die experimentelle Überdosis wurde zusätzlich gewählt, um zu beobachten, ob eventuell bei höheren Bestrahlungsdosen Veränderungen auftreten.

Die Möglichkeit höhere Bestrahlungsdosen für In-vitro-Experimente anzuwenden, wurde auch von al-Nawas und Mitarbeitern (al-Nawas et al. 2000) genutzt. Sie kamen sogar zu dem Schluss, dass die mechanischen Eigenschaften der Zähne, die in vitro bestrahlt werden, erst ab einer experimentellen Dosis von 500 Gy beeinflusst werden. Diese Unterschiede der Reaktionen der Zähne auf die Bestrahlung bei In-vivo- und In-vitro-Methoden erklärten sich al-Nawas mit der in vivo vorkommenden strahleninduzierten Xerostomie und den daraus resultierenden Schäden sowie der Dentinvitalität.

In der vorliegenden Arbeit wurde mit einer Einzeldosis und nicht mit fraktionierten Dosen von z.B. 2 Gy an 5 Tagen/Woche bestrahlt. Diese Methode unterscheidet sich von der von Bulucu et al (2009) oder Soares et al. (2011), die in ihren In-vitro-Studien zur Auswirkung von Strahlentherapie auf das Microleakage der Adhäsivsysteme oder die Bruchfestigkeit von wurzelgefüllten Prämolaren, die klinisch ähnliche, konventionelle fraktionierte Bestrahlung vornahmen.

Die Fraktionierung in der Strahlentherapie bei Patienten mit Tumoren im Kopf-HalsBereich geschieht hauptsächlich deshalb, weil dadurch Reparaturprozesse ablaufen können, wobei sich das gesunde Gewebe schneller regeneriert als das kranke Tumorgewebe. 
In Übereinstimmung mit Experten entschieden wir in dieser Untersuchung, da ausschließlich mit avitalen Rinderzahnproben gearbeitet wurde, dass eine Einfachdosis ausreichend und sinnvoll ist.

\subsection{Ergebnisse}

Die Auswertung und statistische Überprüfung unserer Hauptuntersuchung ergab, dass beide Füllungsmaterialien, sowohl Kunststoff wie Amalgam, keine relevanten Veränderungen vor und nach Bestrahlung im Randspaltbereich zeigen. Die Hypothese, wonach für Kopf-und-Halstumorpatienten durch Radiotherapie in diesem Bereich gefährliche Effekte auftreten könnten, konnte somit nicht bestätigt werden. Auf der Basis der von uns angewandten Methodik wurden keine negativen Auswirkungen von ionisierender Strahlung auf die Randqualität der zahnärztlichen Materialien, Kunststoff und Amalgam sowie der Zahnhartsubstanzen beobachtet.

Dieses aussagekräftige Ergebnis konnte sowohl aufgrund unserer sorgfältigen Voruntersuchungen wie vor allem durch einen soliden Versuchsaufbau im zentralen Experiment erreicht werden.

Bezüglich der Voruntersuchungen ist zunächst noch einmal zu erwähnen, dass sie uns dazu führten, die Anlegung der Kavitäten zu perfektionieren. Folglich waren alle Kavitäten identisch in ihrer Ausdehnung im Schmelz und oberflächlichen Dentin. Sodann haben wir uns für die am häufigsten verwendeten Restaurationsmaterialien in der konservierenden Zahnheilkunde entschieden. Diese Füllungsmaterialien wurden streng nach Maßgabe der Hersteller appliziert. Vor allem aber gewährleisteten die Markierungen, dass immer die

gleichen Flächen evaluiert und so eine optimale Vergleichbarkeit der einzelnen Füllungen geschaffen wurde.

Dennoch ist anzumerken, dass die in unserer Studie standardisierten Vorgehensweisen zu Ergebnissen geführt haben, die sich bei In-vivo-Studien an den Restaurationsmaterialien gegebenenfalls nicht hätten zeigen lassen.

Festzuhalten bleibt desungeachtet als bemerkenswert positives Ergebnis unserer Studie, dass, unter den in den Voruntersuchungen getesteten und schließlich für die Hauptuntersuchung festgelegten Versuchsbedingungen, die Röntgenstrahlen den Füllungsmaterialien 
Kunststoff und Amalgam keine für die Tumorpatienten nachteiligen Veränderungen zufügen können.

Zur differenzierteren Erläuterung der Ergebnisse unseres Experiments ist hier noch Folgendes hinzugefügt:

In unserer Studie zeigte sich, dass bis auf eine Amalgamfüllung aus der Gruppe 1 an allen anderen 39 Füllungen weder vor noch nach der Bestrahlung eine Veränderung zu erkennen war. Die Veränderung an dieser einen Füllung dürfte auf eine durch Zufall entstandene Bruchstelle zurückzuführen sein, vermutlich auf die von Hand durchgeführte Füllungstechnik.

Bei der subjektiven klinischen Überprüfung bekamen in Anwendung der Ryge-Kriterien alle Füllungen die Bestnote Alpha. Zwar hakte die Sonde, die visuelle Beurteilung ließ jedoch keine Stufe erkennen. Es konnte mit dem Fisher-Exact-Test mit der freien Statistiksoftware R statistisch nicht gezeigt werden, dass ein Unterschied zwischen den unbehandelten Proben und den mit der jeweiligen Dosis (60 Gy/90 Gy) bestrahlten Proben vorliegt. Auch trat, statistisch gesehen, kein Unterschied zwischen der 60 Gy-Dosis und der experimentellen Überdosis erkennbar hervor. Die $p$-Werte waren sowohl bei den Amalgam wie bei den Kompositfüllungen größer als 0,05 und die Nullhypothesen blieben folglich bestehen. Der Fisher-Exact-Test wurde für die statistische Ermittlung der Daten genutzt. Er hat zudem den Vorteil, dass er auch bei geringem Stichprobenumfang zuverlässige Resultate liefert.

Noch einmal hervorzuheben ist schließlich auch, dass wir uns in unserer Studie nicht mit der Feststellung des positiven Ergebnisses zufrieden gegeben, sondern auch die Limitationen diskutiert haben. Auf diese Weise ist es uns zudem gelungen, alternative Vorgehensweisen für Nachfolgestudien bereits anzuzeigen - wie z.B. die Verwendung weiterer Füllungsmaterialien, den Gebrauch thermischer Wechselbäder oder die quantitative Randanalyse im Raster-Elektronen-Mikroskop in Verbindung mit der Replikatechnik. - Das alles sind Wege, die in weiteren Studien verfolgt werden können. 


\section{Zusammenfassung}

Strahlentherapie ist ein verbreitetes onkologisches Verfahren, das isoliert oder in einem kombinierten Therapiekonzept bei Patienten mit Tumoren im Kopf-Hals-Bereich eingesetzt werden kann. Trotz diagnostischer und technischer Weiterentwicklungen sind immer noch zahlreiche Nebenwirkungen mit der Radiotherapie verbunden. Bisher kaum untersucht sind die Auswirkungen der Radiotherapien auf zahnmedizinische Materialien.

Die vorliegende Arbeit ging von der Hypothese aus, dass ausgewählte dentale Füllungsmaterialien bei Patienten mit Kopf-Hals-Tumoren durch die Röntgenstrahlung in ihrer Qualität und Beständigkeit, speziell im Bereich der Füllungsränder, negativ beeinflusst werden können.

In einem standardisierten und randomisierten Verfahren wurden 40 Kavitäten in Rinderzähne präpariert. 20 Kavitäten wurden mit Amalgam und 20 mit Komposit gefüllt. 20 Füllungen wurden mit einer Dosis von 60 Gray, 20 Füllungen mit 90 Gray bestrahlt. Die Oberflächen und Grenzen zwischen den Füllungsmaterialien und dem Schmelz wurden vor und nach der Bestrahlung an einer reproduzierbaren Position betrachtet und fotografiert. Zusätzlich wurden alle Füllungen einer klinischen Sondierung unterzogen.

Die Auswertung und statistische Überprüfung unserer Studie ergab, dass beide Füllungsmaterialien, weder bei der mikroskopischen noch der klinischen Untersuchung, relevante Veränderungen vor und nach der Bestrahlung im Randspaltbereich zeigten.

Mit der von uns eingesetzten Methode wurden keine negativen Auswirkungen von Röntgenstrahlen im therapeutischen sowie experimentellen Bereich auf die Randqualität der zahnärztlichen Materialien - Kunststoff und Amalgam - und der Zahnhartsubstanzen nachgewiesen. Unsere Hypothese konnte somit nicht bestätigt werden.

Wir haben in unserer Studie zugleich die Limitation der von ihr angewandten experimentellen Methode reflektiert und diskutiert. Somit bietet sie, über ihr für Patienten mit KopfHals-Tumoren zunächst positives Ergebnis hinausgehend, eine gute Grundlage für weitere Studien. Durch Erweiterung der Methode (i.e. Verwendung weiterer Füllungsmaterialien, quantitative Randanalyse im Raster-Elektronen-Mikroskop in Verbindung mit der Replikatechnik oder eine realitätsnähere Simulation der In-vivo-Begebenheiten wie z.B. durch den Einsatz thermischer Wechselbäder) sollten die Ergebnisse abgesichert werden. Unter den gewählten Bedingungen zeigten sich keine visualisierbaren oder sondierbaren Randspaltbedingungen aufgrund von strahlentherapeutischen Maßnahmen. 


\section{Literaturverzeichnis}

Almeida KG, Scheibe KG, Oliveira AE, Alves CM, Costa JF (2009): Influence of human and bovine substrate on the microleakage of two adhesive systems. J Appl Oral Sci 17(2), 9296

al-Nawas B, Grötz KA, Rose E, Duschner H, Kann P, Wagner W (2000): Using ultrasound transmission velocity to analyse the mechanical properties of teeth after in vitro, in situ, and in vivo irradiation. Clin Oral Investig 4 (3), 168-172

Alptekin T, Ozer F, Unlu N, Cobanoglu N, Blatz MB (2010): In vivo and in vitro evaluations of microleakage around Class I amalgam and composite restorations. Oper Dent $\underline{35}(6)$, 641-648

Amaechi BT, Higham SM (2001): In vitro remineralisation of eroded enamel lesions by saliva. J Dent $\underline{29}(5), 371-376$

Andrews N, Griffiths C (2001a): Dental complications of head and neck radiotherapy: Part 1. Aust Dent J $\underline{46}(2), 88-94$

Andrews N, Griffiths C (2001b): Dental complications of head and neck radiotherapy: Part 2. Aust Dent J $\underline{46}(3), 174-182$

Arends J, Jongebloed WL (1978): Crystallites dimensions of enamel. J Biol Buccale $\underline{6}(3)$, 161-171

Asmussen E, Jorgensen KD (1972): A microscopic investigation of the adaptation of some plastic filling materials to dental cavity walls. Acta Odontol Scand $\underline{30}(1), 3-21$

Barnes IE (1978): Replication techniques for the scanning electron microscope. 1. History, materials and techniques. J Dent $\underline{6}(4), 327-341$

Bedwinek JM, Shukovsky LJ, Fletcher GH, Daley TE (1976): Osteonecrosis in patients treated with definitive radiotherapy for squamous cell carcinomas of the oral cavity and naso-and oropharynx. Radiology 119(3), 665-667

Bernier J, Bentzen SM (2003): Altered fractionation and combined radio-chemotherapy approaches: pioneering new opportunities in head and neck oncology. Eur J Cancer 39(5), 560-571

Beumer J, Brady FA (1978): Dental management of the irradiated patient. Int J Oral Surg $\underline{7}(3), 208-220$

Biscaro SL, Moraes RR, Correr AB, Almeida SM, Bóscolo FN, Soares CJ, Correr-Sobrinho L (2009): Effect of X-ray radiation dose on the bond strength of different adhesive systems to dentin. J Adhes Dent 11(5), 355-360

Blunck U, Roulet JF (1989): In vitro marginal quality of dentin-bonded composite resins in Class V cavities. Quintessence Int 20(6), 407-412

Blunck U, Pohl M, Dieker A (1990): Extraktionszeitpunkt und Kavitätenlage bei der Invitro-Prüfung von Dentinhaftmitteln. Dtsch Zahnarztl Z $\underline{45}$ (10), 639-642

Bonan PR, Lopes MA, Pires FR, Almeida OP (2006): Dental management of low socioeconomic level patients before radiotherapy of the head and neck with special emphasis on the prevention of osteoradionecrosis. Braz Dent J 17(4), 336-342 
Bornstein M, Filippi A, Buser D (2001): Früh- und Spätfolgen im intraoralen Bereich nach Strahlentherapie. Schweiz Monatsschr Zahnmed 111(1), 61-73

Bulucu B, Avsar A, Demiryürek EO, Yesilyurt C (2009): Effect of radiotherapy on the microleakage of adhesive systems. J Adhes Dent 11(4), 305-309

Cawley MM, Benson LM (2005): Current trends in managing oral mucositis. Clin J Oncol Nurs $\underline{9}(5), 584-592$

Chin DW, Treister N, Friedland B, Cormack RA, Tishler RB, Makrigiorgos GM, Court LE (2009): Effect of dental restorations and prostheses on radiotherapy dose distribution: a Monte Carlo study. J Appl Clin Med Phys 10(1), 2853

Collins CJ, Bryant RW, Hodge KL (1998): A clinical evaluation of posterior composite resin restorations: 8-year findings. J Dent 26(4), 311-317

Crim GA, Mattingly SL (1981): Evaluation of two methods for assessing marginal leakage. J Prosthet Dent $\underline{45}(2), 160-163$

Crim GA, Swartz ML, Phillips RW (1985): Comparison of four thermocycling techniques. J Prosthet Dent $\underline{53}(1), 50-53$

Curi MM, Dib LL, Pinto DS (1997): Management of solid ameloblastoma of the jaws with liquid nitrogen spray cryosurgery. Oral Surg Oral Med Oral Pathol Oral Radiol Endod 84(4), 339-344

Cvar JF, Ryge G (2005): Reprint of criteria for the clinical evaluation of dental restorative materials. 1971. Clin oral Investig $\underline{9}(4), 215-232$

Davidson CL, Boom G, Arends J (1973): Calcium distribution in human and bovine surface enamel. Caries Res $\underline{7}(4), 349-359$

De Moor RJ, Stassen IG, van 't Veldt Y, Torbeyns D, Hommez GM (2011): Two-year clinical performance of glass ionomer and resin composite restorations in xerostomic head-and neck-irradiated cancer patients. Clin Oral Investig 15(1), 31-38

de Siqueira Mellara T, Palma-Dibb RG, de Oliveira HF, Garcia Paula-Silva FW, Nelson-Filho P, da Silva RA, da Silva LA, de Queiroz AM (2014): The effect of radiation therapy on the mechanical and morphological properties of the enamel and dentin of deciduous teethan in vitro study. Radiat Oncol $\underline{9}(1), 30$

Devlin H, Bassiouny MA, Boston D (2006): Hardness of enamel exposed to Coca-Cola and artificial saliva. J Oral Rehabil $\underline{33}(1), 26-30$

DGZMK (2002): Wissenschaftliche Stellungnahme: Zahnärztliche Betreuung von Patienten mit tumortherapeutischer Kopf-Hals-Bestrahlung

http://www.dgzmk.de/uploads/tx_szdgzmkdocuments/20020901_Zahnaerztliche_Betreu ung_von_Patienten_mit_tumortherapeutischer.pdf [Zugriff am 01.05.2015]

Dörr W, Hamilton CS, Boyd T, Reed B, Denham JW (2002): Radiation-induced changes in cellularity and proliferation in human oral mucosa. Int J Radiat Oncol Biol Phys $\underline{52}(4), 911-$ 917

Dreizen S, Daly TE, Drane JB, Brown LR (1977): Oral complications of cancer radiotherapy. Postgrad med 61(2), 85-92

Duncalf WV, Wilson NH (2001): Marginal adaptation of amalgam and resin composite restorations in Class II conservative preparations. Quintessence Int $\underline{32(5), 391-395}$ 
Dünninger P, Einwag J, Sitter H (1991): Reproduzierbarkeit von Messungen zur Ergebnisqualität zahnärztlicher Füllungen. Dtsch Zahnarztl Z $\underline{46}(3), 212-214$

Eilers J, Epstein JB (2004): Assessment and measurement of oral mucositis. Semin Oncol Nurs $\underline{20}(1), 22-29$

Engelmeier RL, King GE (1983): Complications of head and neck radiation therapy and their management. J Prosthet Dent $\underline{49}(4), 514-522$

Farahani M, Eichmiller FC, McLaughlin WL (1990): Measurement of absorbed doses near metal and dental material interfaces irradiated by $\mathrm{x}$ - and gamma-ray therapy beams. Phys Med Biol 35(3), 369-385

Farahani M, Eichmiller FC, McLaughlin WL (1993): New method for shielding electron beams used for head and neck cancer treatment. Med Phys 20(4), 1237-1241

Farrington M, Cullen L, Dawson C (2010): Assessment of oral mucositis in adult and pediatric oncology patients: an evidence-based approach. ORL Head Neck Nurs $\underline{28}(3), 8-15$

Ferlay J, Shin HR, Bray F, Forman D, Mathers C, Parkin DM (2010): Estimates of worldwide burden of cancer in 2008: GLOBOCAN 2008. Int J Cancer 127(12), 2893-2917

Ferracane JL (2011): Resin composite-state of the art. Dent Mater 27(1), 29-38

Feyer P, Buchali A (2007): Strahlentherapie. best practice onkologie 2 (5), 42-57

Fonseca RB, Haiter-Neto F, Carlo HL, Soares CJ, Sinhoreti, MA, Puppin-Rontani RM, CorrerSobrinho L (2008): Radiodensity and hardness of enamel and dentin of human and bovine teeth, varying bovine teeth age. Arch Oral Biol 53(11), 1023-1029

Frankenberger R, Tay FR (2005): Self-etch vs etch-and-rinse adhesives: effect of thermomechanical fatigue loading on marginal quality of bonded resin composite restorations. Dent Mater 21(5), 397-412

Frankenberger R, Krämer N, Lohbauer U, Nikolaenko SA, Reich SM (2007): Marginal integrity: is the clinical performance of bonded restorations predictable in vitro? J Adhes Dent 9 Suppl 1, 107-116

Gagnon WF, Cundiff JH (1980): Dose enhancement from backscattered radiation at tissuemetal interfaces irradiated with high energy electrons. Br J Radiol 53 (629), 466-470

Gibbs FA, Palos B, Goffinet DR (1976): The metal/tissue interface effect in irradiation of the oral cavity. Radiology $\underline{119}$ (3), 705-707

Going RE (1972): Microleakage around dental restorations: a summarizing review. J Am Dent Assoc 84(6), 1349-1357

Goldberg J, Tanzer J, Munster E, Amara J, Thal F, Birkhed D (1981): Cross-sectional clinical evaluation of recurrent enamel caries, restoration of marginal integrity, and oral hygiene status. J Am Dent Assoc 102(5), 635-641

Goldstein M, Maxymiw WG, Cummings BJ, Wood RE (1999): The effects of antitumor irradiation on mandibular opening and mobility: a prospective study of 58 patients. Oral Surg Oral Med Oral Pathol Oral Radiol Endod $\underline{88}(3), 365-373$

Gonçalves LM, Palma-Dibb RG, Paula-Silva FW, Oliveira HF, Nelson-Filho P, Silva LA, Queiroz AM (2014): Radiation therapy alters microhardness and microstructure of enamel and dentin of permanent human teeth. J Dent 42(8), 986-992 
Grossman ES, Matejka JM (1997): Amalgam marginal quality assessment: a comparison of seven methods. J Oral Rehabil 24(7), 496-505

Grötz KA, Duschner H, Kutzner J, Thelen M, Wagner W (1997): Neue Erkenntnisse zur Atiologie der sogenannten Strahlenkaries. Nachweis direkter radiogener Veränderungen an der Schmelz-Dentin-Grenze. Strahlenther Onkol 173(12), 668-676

Grötz KA, Duschner H, Kutzner J, Thelen M, Wagner W (1998): Histotomographische Untersuchungen zur Frage direkt radiogener Schmelzveränderungen. Mund Kiefer Gesichtschir $\underline{2}(2), 85-90$

Grötz KA, Riesenbeck D, Brahm R, Seegenschmiedt MH, al-Nawas B, Dörr W, Kutzner J, Willich N, Thelen M, Wagner W (2001): Chronische Strahlenfolgen an den Zahnhartgeweben ("Strahlenkaries"). Klassifikation und Behandlungsansätze. Strahlenther Onkol 177(2), 96-104

Hannig M, Friedrichs C (2001): Comparative in vivo and in vitro investigation of interfacial bond variability. Oper Dent $\underline{26}(1), 3-11$

Haveman CW, Summitt JB, Burgess JO, Carlson K (2003): Three restorative materials and topical fluoride gel used in xerostomic patients: a clinical comparison. J Am Dent Assoc 134(2), 177-184

He Z, Shimada Y, Sadr A, Ikeda M, Tagami J (2008): The effects of cavity size and filling method on the bonding to Class I cavities. J Adhes Dent 10(6), 447-453

Heintze SD (2007): Systematic reviews: I. The correlation between laboratory tests on marginal quality and bond strength. II. The correlation between marginal quality and clinical outcome. J Adhes Dent 9 Suppl 1, 77-106

Hellwig E, Klimek J, Attin T: Einführung in die Zahnerhaltung: Prüfungswissen Kariologie, Endodontologie und Parodontologie mit 60 Tabellen. 5. überarb. und erw. Auflage; Dt. Zahnärzte-Verl., Köln 2009

Henkin RI, Talal N, Larson AL, Mattern CF (1972): Abnormalities of taste and smell in Sjogren's syndrome. Ann Intern Med 76(3), 375-383

Herzog M, Samek M, Siranli F (1986): Zur Indikation der zahnärztlich-chirurgischen Sanierung bei Strahlentherapie. Dtsche Zahnarztl Z $\underline{41}(4)$, 449-451

Hickel R, Manhart J (2001): Longevity of restorations in posterior teeth and reasons for failure. J Adhes Dent $\underline{3}(1), 45-64$

Holan G, Chosack A, Eidelman E (1996): Clinical evaluation of Class II combined amalgamcomposite restorations in primary molars after 6 to 30 months. ASDC J Dent Child $\underline{63}(5)$, 341-345

Hong CH, Napeñas JJ, Hodgson BD, Stokman MA, Mathers-Stauffer V, Elting LS, Spijkervet FK, Brennan MT (2010): A systematic review of dental disease in patients undergoing cancer therapy. Support Care Cancer 18(8), 1007-1021

Horiot JC, Schraub S, Bone MC, Bain Y, Ramadier J, Chaplain G, Nabid N, Thevenot B, Bransfield D (1983): Dental preservation in patients irradiated for head and neck tumours: A 10-year experience with topical fluoride and a randomized trial between two fluoridation methods. Radiother Oncol $\underline{1}(1), 77-82$ 
Hurst D (2014): Amalgam or composite fillings-which material lasts longer? Evid Based Dent $\underline{15}(2), 50-51$

Ikeda T, Uno S, Tanaka T, Kawakami S, Komatsu H, Sano H (2002): Relation of enamel prism orientation to microtensile bond strength. Am J Dent $\underline{15}(2), 109-113$

Jansma J, Buskes J, Vissink A, Mehta DM, Gravenmade EJ (1988a): The Effect of X-Ray Irradiation on the Demineralization of Bovine Dental Enamel. Caries Res 22 (4), 199-203

Jansma J, Vissink A, Gravenmade EJ, de Josselin de Jong, E, Jongebloed WL, Retief DH (1988b): A model to investigate xerostomia-related dental caries. Caries Res 22 (6), 357361

Kaanders JH, Fleming TJ, Ang KK, Maor MH, Peters LJ (1992): Devices valuable in head and neck radiotherapy. Int J Radiat Oncol Biol Phys 23(3), 639-645

Kappert HF, Knode H, Schultheiss R (1991): Festigkeitsverhalten der In-Ceram-Keramik bei mechanischer und thermischer Wechsellast im Kunstspeichel. Dtsch Zahnarztliche Z $\underline{46}(2)$, 129-131

Kauffmann GW, Moser E, Sauer R: Radiologie. Mit 67 Tabellen ; Fragen und Antworten. 2. Auflage; Urban und Fischer, München 2001

Kielbassa AM, Munz I, Bruggmoser G, Schulte-Mönting J (2002): Effect of demineralization and remineralization on microhardness of irradiated dentin. J Clin Dent 13(3), 104-110

Kielbassa AM (Hrsg.): Strahlentherapie im Kopf- und Halsbereich: Implikationen für Zahnärzte, HNO-Ärzte und Radiotherapeuten; Schlütersche, Hannover 2004

Kielbassa AM, Hinkelbein W, Hellwig E, Meyer-Lückel H (2006): Radiation-related damage to dentition. The Lancet Oncol $\underline{7}(4), 326-335$

Klimek J, Hellwig E, Ahrens G (1982): Fluoride taken up by plaque, by the underlying enamel and by clean enamel from three fluoride compounds in vitro. Caries Res 16 (2), 156161

Koga DH, Salvajoli JV, Alves FA (2008): Dental extractions and radiotherapy in head and neck oncology: review of the literature. Oral Dis $\underline{14}(1), 40-44$

Kóhalmi T, Gorzo I, Mari A, Nagy K (1999): In vitro comparison of marginal adaptation of various filling materials. I. Effect of the filling material on marginal adaptation. Fogorv $\mathrm{Sz}$ 92(3), 87-95

Krebs in Deutschland 2009/2010. 9. Ausgabe. Robert Koch-Institut (Hrsg.) und die Gesellschaft der epidemiologischen Krebsregister in Deutschland e.V. (Hrsg.). Berlin 2013

Kreulen CM, van Amerongen WE, Borgmeijer PJ, Akerboom HB (1993): Comparison of two methods for evaluating the occlusal marginal adaptation of posterior restorations. ASDC J Dent Child 60(4-5), 304-309

Kusy RP, Leinfelder KF (1977): In situ replication techniques: I. Preliminary screening and the negative replication technique. J Dent Res 56(8), 925-932

Larson DL (1986): Management of complications of radiotherapy of the head and neck. Surg Clin North Am $\underline{66}(1), 169-182$ 
Maier A, Gaggl A, Klemen H, Santler G, Anegg U, Fell B, Kärcher H, Smolle-Jüttner FM, Friehs GB (2000): Review of severe osteoradionecrosis treated by surgery alone or surgery with postoperative hyperbaric oxygenation. Br J Oral Maxillofac Surg 38(3), 173-176

Mail N, Albarakati Y, Ahmad Khan M, Saeedi F, Safadi N, Al-Ghamdi S, Saoudi A (2013): The impacts of dental filling materials on RapidArc treatment planning and dose delivery: challenges and solution. Med Phys $\underline{40}(8), 081714$

Marx RE (1983): Osteoradionecrosis: a new concept of its pathophysiology. J Oral Maxillofac Surg $\underline{41}(5), 283-288$

McComb D, Erickson RL, Maxymiw WG, Wood RE (2002): A clinical comparison of glass ionomer, resin-modified glass ionomer and resin composite restorations in the treatment of cervical caries in xerostomic head and neck radiation patients. Oper Dent $\underline{27}(5), 430$ 437

Mealey BL, Semba SE, Hallmon WW (1994): The head and neck radiotherapy patient: Part 2-Management of oral complications. Compendium 15(4), 442, 444, 446-52 passim; quiz 458

Melian E, Fatyga M, Lam P, Steinberg M, Reddy SP, Petruzzelli GJ, Glasgow GP (1999): Effect of metal reconstruction plates on cobalt- 60 dose distribution: a predictive formula and clinical implications. Int J Radiat Oncol Biol Phys 44 (3), 725-730

Mitchell RJ, Koike M, Okabe T (2007): Posterior amalgam restorations--usage, regulation, and longevity. Dent Clin North Am 51(3), 573-89

Möller P, Perrier M, Ozsahin M, Monnier P (2004): A prospective study of salivary gland function in patients undergoing radiotherapy for squamous cell carcinoma of the oropharynx. Oral Surg Oral Med Oral Pathol Oral Radiol Endod 97(2), 173-189

Moore S, Burke MC, Fenlon MR, Banerjee A (2012): The role of the general dental practitioner in managing the oral care of head and neck oncology patients. Dent Update $\underline{39}(10)$, 694-6, 698-700, 702

Mossman KL (1986): Gustatory tissue injury in man: radiation dose response relationships and mechanisms of taste loss. Br J Cancer Suppl $\underline{7}, 9-11$

Murdoch-Kinch CA, Zwetchkenbaum S (2011): Dental management of the head and neck cancer patient treated with radiation therapy. J Mich Dent Assoc 93(7), 28-37

Nakamichi I, Iwaku M, Fusayama T (1983): Bovine Teeth as Possible Substitutes in the Adhesion Test. J Dent Res 62 (10), 1076-1081

Naves LZ, Novais VR, Armstrong SR, Correr-Sobrinho L, Soares CJ (2012): Effect of gamma radiation on bonding to human enamel and dentin. Support Care Cancer 20(11), 28732878

Noack MJ (1988): Quantitative Füllungsrandanalyse von Frontzahnkompositen im RasterElektronen-Mikroskop nach thermischer Wechselbelastung. Dtsch Zahnarztl Z $\underline{43}(3), 295-$ 299

O'Dell K, Sinha U (2011): Osteoradionecrosis. Oral Maxillofac Surg Clin North Am 23(3), 455-464

Oesterle L, Shellhart WC, Belanger GK (1998): The use of bovine enamel in bonding studies. Am J Orthod Dentofacial Orthop 114(5), 514-519 
Olthoff A, Ewen A, Wolff HA, Hermann RM, Vorwerk H, Hille A, Rödel R, Hess CF, Steiner W, Pradier O, Christiansen $H$ (2009): Organ function and quality of life after transoral laser microsurgery and adjuvant radiotherapy for locally advanced laryngeal cancer. Strahlenther Onkol 185(5), 303-309

Ozer F, Blatz MB (2013): Self-etch and etch-and-rinse adhesive systems in clinical dentistry. Compend Contin Educ Dent 34(1), 12-4, 16, 18; quiz 20, 30

Palotie U, Vehkalahti M (2002): Reasons for replacement and the age of failed restorations in posterior teeth of young Finnish adults. Acta odontol Scand $\underline{60}(6), 325-329$

Pashley DH (1991): In vitro simulations of in vivo bonding conditions. Am J Dent $\underline{4}(5), 237-$ 240

Peumans M, De Munck J, Van Landuyt KL, Poitevin A, Lambrechts P, Van Meerbeek B (2012): A 13-year clinical evaluation of two three-step etch-and-rinse adhesives in noncarious class-V lesions. Clin oral investig 16(1), 129-137

Pioch T, Möller D, Staehle HJ, Hoppe W (1991): Über das Lösungsverhalten von Zahnschmelz und synthetischem Hydroxylapatit nach Bestrahlung. Dtsch Zahnarztl Z $\underline{46}(6)$, 413-415

Rahman S, Maillou P, Barker D, Donachie M (2013): Radiotherapy and the oral environment the effects of radiotherapy on the hard and soft tissues of the mouth and its management. Eur Journal Prosthodont Restor Dent 21(2), 80-87

Rammelsberg P, Eickemeyer G, Erdelt K, Pospiech P (2000): Fracture resistance of posterior metal-free polymer crowns. J Prosthet Dent 84(3), 303-308

Rasines Alcaraz MG, Veitz-Keenan A, Sahrmann P, Schmidlin PR, Davis D, Iheozor-Ejiofor Z (2014): Direct composite resin fillings versus amalgam fillings for permanent or adult posterior teeth. Cochrane Database Syst Rev $\underline{3}$, CD005620

Regezi JA, Courtney RM, Kerr DA (1976): Dental management of patients irradiated for oral cancer. Cancer $\underline{38}(2)$, 994-1000

Reichart PA: Curriculum Zahn-, Mund- und Kieferkrankheiten: Diagnostik, spezifische Entzündungen, Herderkrankungen, Zysten, Erkrankungen der Kiefer, der Kieferhöhle, der Mundschleimhaut der Speicheldrüsen und der Kiefergelenke, Tumoren (Curriculum Chirurgie Band 2); Quintessenz-Verlag, Berlin 2002

Reis AF, Giannini M, Kavaguchi A, Soares CJ, Line SR (2004): Comparison of microtensile bond strength to enamel and dentin of human, bovine, and porcine teeth. J Adhes Dent 6(2), 117-121

Reitemeier B, Reitemeier G, Schmidt A, Schaal W, Blochberger P, Lehmann D, Herrmann T (2002): Evaluation of a device for attenuation of electron release from dental restorations in a therapeutic radiation field. J Prosthet Dent 87(3), 323-327

Rice N, Polyzois I, Ekanayake K, Omer O, Stassen LF (2015): The management of osteoradionecrosis of the jaws - A review. Surgeon $\underline{13}(2), 101-109$

Rodrigues LK, Cury JA, Nobre dos Santos M (2004): The effect of gamma radiation on enamel hardness and its resistance to demineralization in vitro. J Oral Sci $\underline{46}(4), 215-220$

Roulet JF, Reich T, Blunck U, Noack M (1989): Quantitative margin analysis in the scanning electron microscope. Scanning Microsc $\underline{3}(1), 147-58$; discussion 158-9 
Roulet JF (1997): Benefits and disadvantages of tooth-coloured alternatives to amalgam. J Dent $\underline{25}(6), 459-473$

Ryge G, Jendresen MD, Glantz PO, Mjör I (1981): Standardization of clinical investigators for studies of restorative materials. Swed Dent J $\underline{5}(5-6), 235-239$

Schilke R, Lisson JA, Bauss O, Geurtsen W (2000): Comparison of the number and diameter of dentinal tubules in human and bovine dentine by scanning electron microscopic investigation. Arch Oral Biol $\underline{45}(5), 355-361$

Schwenzer N, Ehrenfeld M: Mund-Kiefer-Gesichtschirurgie. 48 Tabellen (Zahn-MundKiefer-Heilkunde [2]). 4., vollst. überarb. und erw. Auflage; Thieme, Stuttgart [u.a.] 2011

Sennhenn-Kirchner S, Freund F, Grundmann S, Martin A, Borg-von Zepelin M, Christiansen H, Wolff HA, Jacobs HG (2009): Dental therapy before and after radiotherapy--an evaluation on patients with head and neck malignancies. Clin Oral Investig 13(2), 157-164

Shannon IL, Wescott WB, Starcke EN, Mira J (1978): Laboratory study of cobalt-60irradiated human dental enamel. J Oral Med $\underline{33}(1), 23-27$

Soares CJ, Roscoe MG, Castro CG, Santana FR, Raposo LH, Quagliatto PS, Novais VR (2011): Effect of gamma irradiation and restorative material on the biomechanical behaviour of root filled premolars. Int Endod J 44(11), 1047-1054

Sperandio M, Souza JB, Oliveira DT (2001): Effect of gamma radiation on dentin bond strength and morphology. Braz Dent J 12(3), 205-208

Spielman Al (1998): Chemosensory function and dysfunction. Crit Rev Oral Biol Med $\underline{9}(3)$, 267-291

Suwelack D, Haverkamp U, Eich HT, Dirksen D, Figgener L, Wolowski A, Runte C (2014): Rückstreueffekte prothetischer Biomaterialen bei der Bestrahlung im Kopf-/Halsbereich. DZZ 69(7), 406-414

Taylor MJ, Lynch E (1992): Microleakage. J Dent 20(1), 3-10

Thiel HJ (1989a): Die Osteoradionekrose. Teil I: Atiologie, Pathogenese, Klinik und Risikofaktoren. Radiobiol Radiother (Berl) 30(5), 397-413

Thiel HJ (1989b): Die Strahlenkaries. Radiobiol Radiother (Berl) 30(3), 193-211

Thilmann C, Adamietz IA, Rahn R, Mose S, Saran F, Schopohl B, Böttcher HD (1995): Invivo-Bestimmung der Dosiserhöhung durch Dentallegierungen bei therapeutischer Bestrahlung der Mundhöhle. Strahlenther Onkol 171(8), 468-472

Tomita Y, Osaki T (1990): Gustatory impairment and salivary gland pathophysiology in relation to oral cancer treatment. Int J Oral Maxillofac Surg 19(5), 299-304

van Meerbeek B, Vargas M, Inoue S, Yoshida Y, Perdigão J, Lambrechts P, Vanherle G (2000): Microscopy investigations. Techniques, results, limitations. Am J Dent $\underline{13}$ (Spec No), 3D-18D

Vissink A, Burlage FR, Spijkervet FK, Jansma J, Coppes RP (2003a): Prevention and treatment of the consequences of head and neck radiotherapy. Crit Rev Oral Biol Med 14(3), 213-225

Vissink A, Jansma J, Spijkervet FK, Burlage FR, Coppes RP (2003b): Oral sequelae of head and neck radiotherapy. Crit Rev Oral Biol Med 14(3), 199-212 
Walker MP, Wichman B, Cheng A, Coster J, Williams KB (2011): Impact of Radiotherapy Dose on Dentition Breakdown in Head and Neck Cancer Patients. Pract Radiat Oncol 1 (3), 142-148

Wang RR, Pillai K, Jones PK (1996): In vitro backscattering from implant materials during radiotherapy. J Prosthet Dent $\underline{75}$ (6), 626-632

Wang Y, Spencer P (2005): Evaluation of the interface between one-bottle adhesive systems and dentin by Goldner's trichrome. Am J Dent 18(1), 66-72

Wijers OB, Levendag PC, Braaksma MM, Boonzaaijer M, Visch LL, Schmitz PI (2002): Patients with head and neck cancer cured by radiation therapy: a survey of the dry mouth syndrome in long-term survivors. Head Neck 24(8), 737-747

Wolff HA, Overbeck T, Roedel RM, Hermann RM, Herrmann MK, Kertesz T, Vorwerk H, Hille A, Matthias C, Hess CF, Christiansen H (2009): Toxicity of daily low dose cisplatin in radiochemotherapy for locally advanced head and neck cancer. J Cancer Res Clinical Oncol 135(7), 961-967

Wolff HA, Wolff CR, Hess CF, Jung K, Sennhenn-Kirchner S, Hinterthaner M, MüllerDornieden A, Körber W, Marten-Engelke K, Roedel R, Christiansen H, Engelke C (2013): Second primary malignancies in head and neck cancer patients: high prevalence of curable-stage disease. Strahlenther Onkol 189(10), 874-880

Wood RE, Maxymiw WG, McComb D (1993): A clinical comparison of glass ionomer (polyalkenoate) and silver amalgam restorations in the treatment of Class 5 caries in xerostomic head and neck cancer patients. Oper Dent 18(3), 94-102

Wöstmann B, Lütke-Notarp E (1991): Wie randständig ist eine "durchschnittliche" Amalgamfüllung? Dtsch Zahnarztl Z $\underline{46}(1), 28-32$

Yassen GH, Platt JA, Hara AT (2011): Bovine teeth as substitute for human teeth in dental research: a review of literature. J Oral Sci $\underline{53}(3), 273-282$ 


\section{Danksagung}

Zuallererst danke ich ganz besonders Frau PD Dr. Sabine Sennhenn-Kirchner, dass sie mir das Thema der Dissertation überlassen hat und mir in allen Phasen der Promotion stets mit einer sehr persönlichen Betreuung und viel Herzlichkeit zur Seite stand.

Außerdem gilt mein Dank Herrn PD Dr. med. Hendrik Wolff sowie Frau Margret RaveFränk aus der Strahlentherapie und Radioonkologie der UMG für ihr Interesse und ihre Bereitschaft, unsere Arbeit zu unterstützen, sowie für die Bereitstellung der experimentellen Röntgenstrahlanlage für die Bestrahlung unserer Proben. Meinen Dank aussprechen möchte ich auch Herrn Dr. rer. nat. Dipl. Biol. Hans Joachim Rolf für seine tatkräftige Unterstützung am Mikroskop, und auch dem Laborteam, ganz besonders den Medizinisch-technischen Assistentinnen Frau Monika Hoch und Frau Christina Schäfer, für ihre freundliche Hilfe während der gesamten Zeit.

Auch die gute und freundschaftliche Zusammenarbeit mit meiner Doktorandenkollegin Frau Katja Schellenberger soll an dieser Stelle nicht unerwähnt bleiben.

Nicht zuletzt bedanke ich mich bei der Fleischerei Sebert's in Göttingen-Geismar, die uns freundlicherweise die Rinderzähne als Probenmaterial zur Verfügung stellte. 